Portland State University

PDXScholar

\title{
Empathy, holonomic brain processes and Patanjali's \\ Sutras : a study of Western and Eastern models of perception as they relate to empathic communication
}

Dana Ralston McCullough

Portland State University

Follow this and additional works at: https://pdxscholar.library.pdx.edu/open_access_etds

Part of the Communication Commons

Let us know how access to this document benefits you.

\section{Recommended Citation}

McCullough, Dana Ralston, "Empathy, holonomic brain processes and Patanjali's Sutras : a study of Western and Eastern models of perception as they relate to empathic communication" (1991).

Dissertations and Theses. Paper 4203.

https://doi.org/10.15760/etd.6087

This Thesis is brought to you for free and open access. It has been accepted for inclusion in Dissertations and Theses by an authorized administrator of PDXScholar. Please contact us if we can make this document more accessible: pdxscholar@pdx.edu. 
AN ABSTRACT OF THE THESIS OF Dana Ralston MCCullough for the Master of Science in Speech Communication presented May 29, 1991.

Title: Empathy, Holonomic Brain Processes and Patanjali's Sutras: A Study of Western and Eastern Models of Perception as They Relate to Empathic Communication.

\section{APPROVED BY THE MEMBERS OF THE THESIS COMMITTEE:}
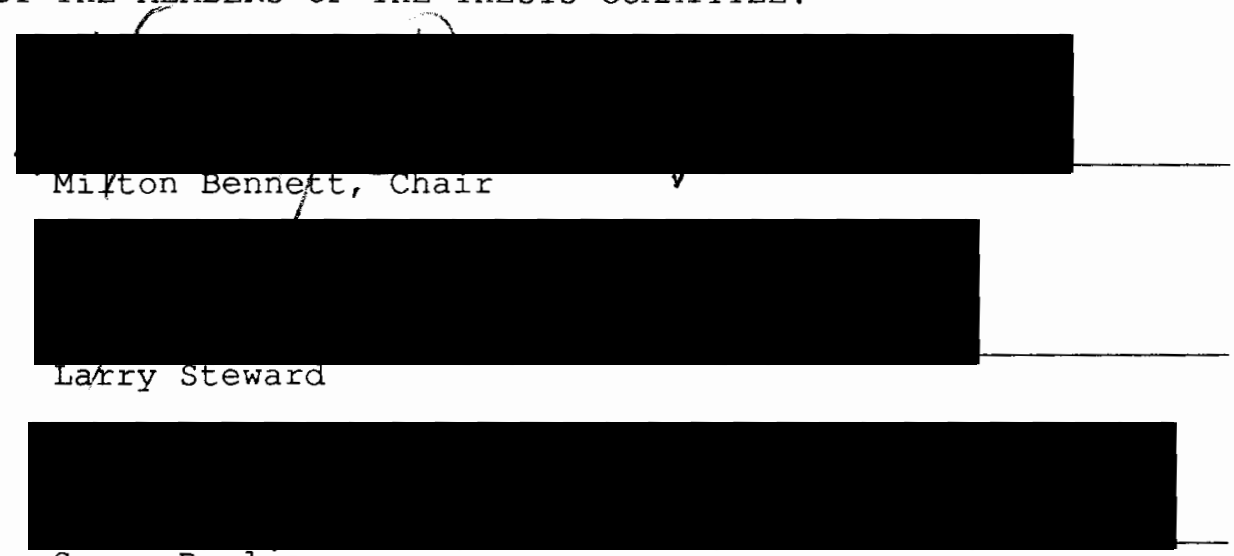

Susan Poulsen

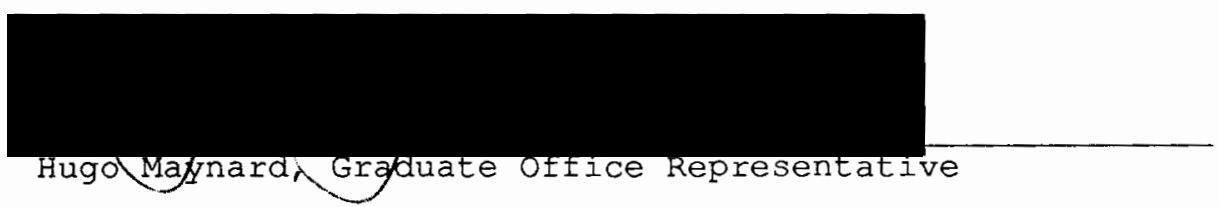

Communication scholars, psychologists, teachers and social

scientists recognize the importance of empathy to effective

communication; however, its essential nature remains a mystery. The nonverbal and subjective aspects of empathic perception make it difficult to study with traditional Western scientific tools. Existing studies of empathic process concentrate primarily on the early or the latter stages. The central steps are seldom addressed. A method is needed for following empathic perception to its core processes and 
exploring the principles that govern its nature. Systems science offers a viable alternative method of studying empathy through analysis of models, emphasizing principles of interaction and process.

In this thesis, empathic principles are explored through the perceptual potentials inherent in Dr. Karl Pribram's holonomic brain model and Patanjali's Yoga Sutras. The central concept of isomorphism as it relates to direct attunement is examined from both perspectives-Western science and Eastern experiential knowledge. First, the hologram is defined and explored as an explanatory model for human perception. Then holonomic perception is described and examined for specific principles of process, interaction and integration that shed light on the direct empathic perception. Among these principles are holism, isomorphism, creation of constancies, enfolding and unfolding of process to form and form to process, intuition, synchronizing to sense invariants, nonlinear correlations and interrelationships between the three domains of constructional realism. The holonomic paradigm alters the concept of dualism by subsuming it in a larger context that includes both an unfolded and an enfolded/distributed domain. The enfolded/distributed domain corresponds to the implicate order described by physicist David Bohm. Empathic attention is found to play a central, communicating role between both domains. The second model, Patanjali's Sutras, formulates specific attentional considerations for achieving the isomorphic cohenence necessary to this communicating role. Modifications of the mind that inhibit isomorphic perception are examined. Isomorphism, samadhi, and direct knowing are found to be 
consonant terms. Empathy is identified as one perceptual capacity in a larger continuum of the human potential for direct perception--from objects, to people, to the mind.

With the new perspectives and insights from these models, basic assumptions about human communication are recontextualized, and empathic communication is redefined. Theodore Reik's four stages of empathy are expanded, taking into account the interactive principles of holonomic perception and the enhanced role of empathic reverberation and attunement. 
EMPATHY, HOIONOMIC BRAIN PROCESSES AND PATANJALI'S SUTRAS:

A STUDY OF WESTERN AND EASTERN MODELS OF

PERCEPTION AS THEY RELATE TO

EMPATHIC COMMUNICATION

by

DANA RALSTON MCCULLOUGH

A thesis submitted in partial fulfillment of the requirements for the degree of

MASTER OF SCIENCE
in
SPEECH COMMUNICATION

Portland State University

1991 
TO THE OFFICE OF GRADUATE STUDIES:

The members of the Committee approve the thesis of Dana Ralston McCullough presented May 29, 1991.
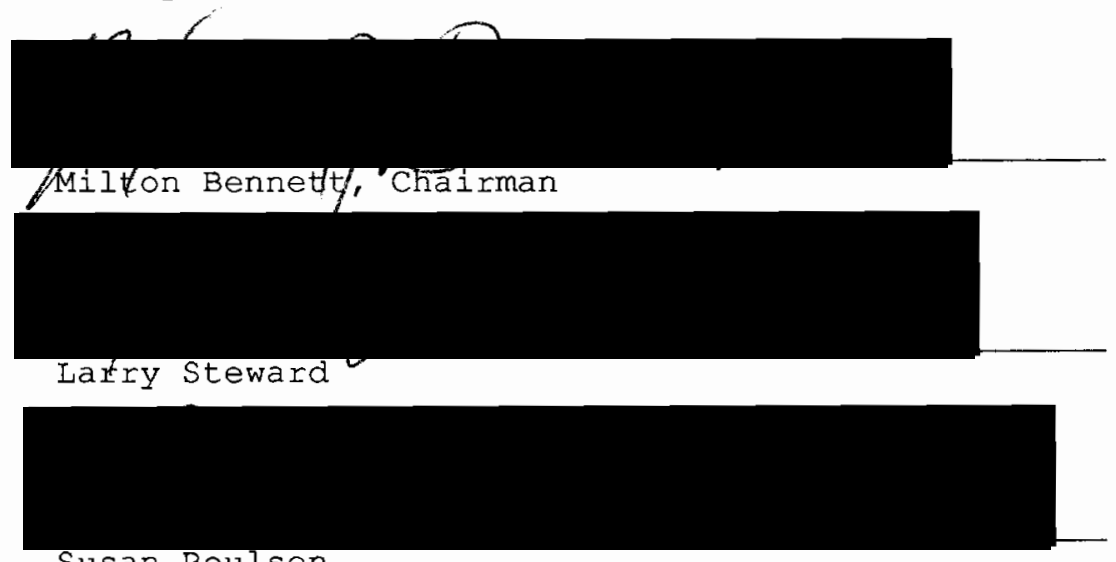

Susan Poulsen

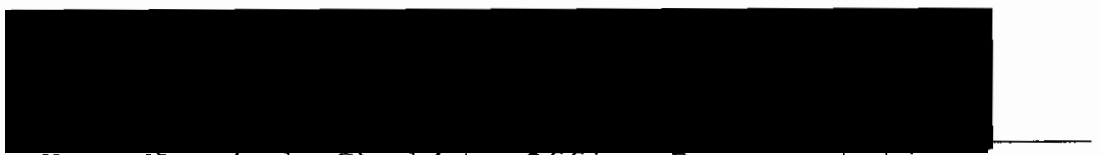

Hugo Mypard, Gtacyate office Representative

APPROVED :

Theodore Grove, Chalr, Department of speech communication

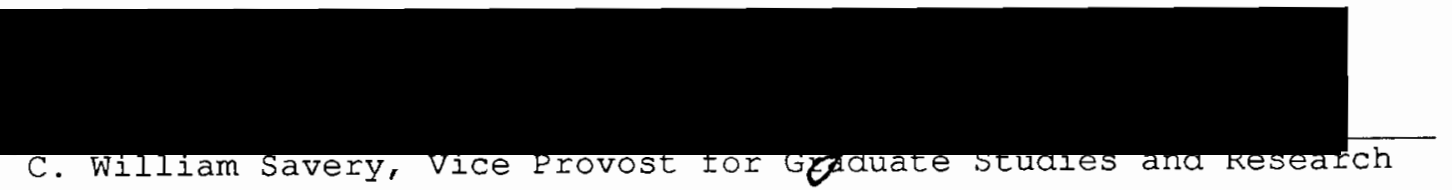


PREFACE

Years ago, I added a unit on empathy to my high school public speaking classes. As I taught empathy, I discovered I knew little about it and became intrigued with the subject. Later, this interest increased when I sat at the Republican National Convention and with amazement and no small discomfort watched Richard Nixon--using communication skills that evoked empathic response--take a mere 20 minutes to transform hundreds of tired, discouraged and embittered delegates into a thunderous hall, screaming support for a heretofore unpopular, far-right candidate. Still later, while traveling in Malaysia, I looked into the eyes of an Asian man whom I had inadvertently insulted and felt a psychological wallop, then disorientation and nausea from suddenly feeling his loss of face--an emotion that I, as a Westerner, never appreciated before. The surprise and intensity of this experience have never left me. Closing my eyes, I can relive it through seeing his. I have watched with wonder, too, as the eyes of an empathic listener eased the inner states of a troubled confidant. It also seems that small children, as a whole, have heightened ability to merge inwardly with those they watch, enhancing, I believe, their learning efficiency and preparing ground for future interactions.

What is empathy, really? As the examples show, it can be consciously or unconsciously experienced, used or abused. Attention to the subject could move us in the direction of responsibility, awareness 
and appreciation for its capacity to enrich our lives and improve our communicative skills.

However, first we have to understand it.

I thank Dr. Milton Bennett, my thesis advisor, and Portland state University's Department of Speech Communication for the opportunity to pursue a creative thesis on empathic perception through models and synthesis rather than using a traditional reductionist approach. To the best of my knowledge, the connection between empathic perception and these models has not yet been explored in the literature. Karl Pribram's gracious response in granting three lengthy interviews on the subject, and Shyam Gosh's encouragement from 7000 miles away were also indispensable to its design and completion. 
TABLE OF CONTENTS

PAGE

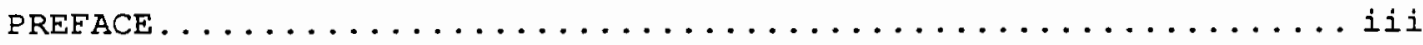

IIST OF EIGURES...........................

CHAPTER

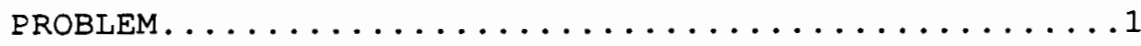

Empathy Defined as Perceptual Process.........

Cultural and Scientific Biases That Inhibit

Study of Empathic Process...........

II

METHOD ........................

Systems Science as an Alternative Approach....10

Choosing Appropriate Models for Empathic.....15 Process

III

HOIONOMIC PERCEPTION AND EMPATHY...........24

The Holonomic Model.................24

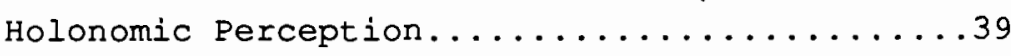

Application of Principles of Holonomic

Perception to Empathic Process.........51

Attention and Empathic Perception.........89

IV PATANJALI'S YOGA SUTRAS AND EMPATHY..........92

Background.......................

Selected Sutras That Relate to Empathic

Perception...................95

Isomorphism, Samadhi and Direct Perception...109

Empathic Perception via Awareness

in a Lensless domain...............119 
New Paradigm Perspectives: General

Implications for Communication

and Empathy................... 127

Reik Revised: Applying the Principles of Holonomic Empathic Perception to

Everyday Life......................

SOURCES CONSULTED........................... 138

APPENDICES

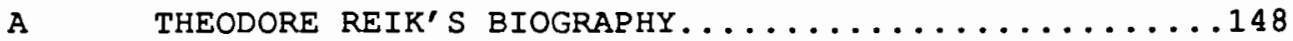

B BRIEF HISTORY OF EMPATHY AS A CONSTRUCT.......... 151

C DR. KARL H. PRIBRAM'S BIOGRAPHY AND RESEARCH..... 155

D CHRONOLOGY OF THE HOLONOMIC BRAIN METAPHOR......160

E HOLOGRAM MODEL AND EXPLANATION ............ 162

F FOURIER TRANSFORM: HISTORY, THEOREM AND

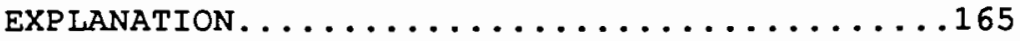

G HOLONOMIC ENFOLDING-UNFOLDING SCHEMATIC........169 


\section{LIST OF FIGURES}

FIGURE

PAGE

Representative Neurons..................41

Neuron and Dendrites...................42

Arborization and Thickening of Dendrites in

Children 1 Month, 6 Months and 2 Years Old...44

4

Constructional Realism: A Schematic Depicting

Possible Perceptual Interactions..........53

Isomorphism="Same Form" .................113

6

Samadhi=Direct Perception...............114 


\section{CHAPTER I}

\section{PROBLEM}

For one person to relate effectively to another, one needs an accurate sense of the other's inner states and ongoing reactions (Katz, 1963, p. 15). Without this, at some point communication breaks down. The interpersonal, family, institutional, community, national and international cost of failed understanding is immeasurable. Human experience is diminished by failed communication. Isolation, dehumanization, inefficiency, missed opportunity, confusion, and even war can be cumulative consequences of the inability to "read" and respond to the nonverbal context of another's message.

Some theorists see empathy as a complement to logic and reasoning for intuiting the inner nature of another human, thus mitigating the possibilities of failed communication. Deutsch and Madle (1975) observe empathy as "an ability to understand a person's emotional reactions in consort with the context" (p. 270). Katz (1963) takes the observation farther, explaining:

Why must the student of human personalities become empathically involved with his subjects? . . Fliess, Levine, and others recommend the use of empathy because this technique leads to "inside knowledge that is almost firsthand" and provides an "immediate comprehension" that in some respects may be superior even to intellectual understanding. If we study human personality logically and intellectually, an immediate and direct grasp of the inner core of the person is likely to elude us. We are unable to appreciate the subtleties and nuances of his emotions. . . Logic also disappoints us if we intend to study a particular human personality in its uniqueness and individuality. Reason is most useful when we assign the individual to a category. It 


\begin{abstract}
falls wide of the maxk when we aim to understand a man not in terms of his similarities with others but in terms of his own personal and private characteristics. The case for empathy rests on the fact that however similar human beings may be, there is something distinctive and unique about each. If our goal is to understand what is individual and distinctive in a person we must use empathic methods. (pp. 14-15)
\end{abstract}

As it is discussed in this thesis, empathic perception may enable us to make the unknown known, to develop an affinity for or understanding of the other person that is encoded directly into our neural apparatus. Through empathic perception we may have the capacity to "real-ize" the other person with our being. According to the concept of empathy used here, this communication is felt directly giving richer context to words uttered. Thus, when the inner "dance" of attunement enables us to feel in harmony with another's emotional, intentional and energy states, we have accomplished an essential aspect of of communicating--even if the words exchanged are superficial or at variance with the unspoken context.

Each person, each culture, has codes of learned behavior and constructs which impact this inner attunement, facilitating or buffering the process (Condon \& Yousef, 1975; Hall, 1959; Sapir, 1958; Whorf, 1956). However, if we could go deeper and know more about the empathic process itself, we might enrich all levels of communication by listening more sensitively for communicative dissonance.

In his article, "Empathic: An Unappreciated Way of Being," Carl Rogers (1975) addresses these empathic potentials when he observes, "the empathic process can be defined in theoretical, conceptual, subjection 
and operational ways. Even so, we have not reached the limits of its base" (p.4).

The purpose of this thesis is to study empathic process by inferring its base. Two disparate frames of reference will be brought to bear on the concept of empathy. One, a Western perspective, will probe certain neuropsychological processes for their relevance to the base of empathy. The other, an Eastern perspective, will search certain Yoga Sutras for similar relevance. Specifically, the research question is: What can be learned about empathic perception from a combined perspective of Pribram's holonomic brain model and Patanjali's Yoga Sutras?

To address the research question this thesis will examine Karl Pribram's (1971) 1 empirical reframing of human perception--the cornerstone of the "holographic paradigm"--and it will explore a blueprint for "correct use of perception"--Patanjali's Sutras"--to discover how each may apply to and illuminate the subjective experience of empathic attunement, as described by Theodore Reik (1948).

\section{EMPATHY DEFINED AS PERCEPTUAL PROCESS}

Throughout this thesis, the term empathy refers to a process rather than a state. "Process involves one state becoming another" (Pribram, 1982b, p. 251). The thesis will explore the contention that humans share a universal ability to attune directly to another's inner affective states; or, phrased alternatively, that we can establish accurate nonverbal context for communication by allowing ourselves to 
resonate to another's feelings, intentions and energy level. The process involved has structure, and here structure "is not be confused with morphology or anatomical structure. Structure here means the structure of process, the meaning used by Hertz and Levi-Stauss (1963) and by Merleau-Ponty (1963)" (Pribram, 1982b, p. 251).

This paper will reference Theodore Reik's (1948) generally accepted experiential description of empathic process. Reik, a student of Sigmund Freud, based his definition on insights from over 30 years of psychoanalytic listening (Appendix A). His four phases of empathy, described by Katz (1963) in Empathy: Its Nature and Uses, are summarized below:

1. Identification: Allowing oneself to become engrossed in the personality of another and lose consciousness of self. "Our entire consciousness is projected into another person so that the feelings that inhere in others act upon us" (p. 42).

2. Incorporation: The act of taking the experience of the other person into one's own experience.

3. Reverberation: Allowing the other person's reality to comingle with one's own. Part of the self is fused with the identity of the other. Yet another part of one's feeling and thinking is capable of responding to this experience as the external thing it is. One remains involved, attached, and identified long enough to allow for personal reactions to be evoked and for deeper levels of one's own unconscious to be stimulated. 
4. Detachment: To withdraw from subjective involvement and gain the social and psychic distance necessary for objective analysis (pp. $41-47)$.

The study will focus on steps two and three, the nonverbal incorporation and reverberation aspects of Reik's (1948) overall description. They represent his effort to describe the base event; the first and last steps move toward or away from that event. Reik repeatedly tells his readers that the process cannot be divided into steps and then approached linearly. He, too, was most interested in the central aspects:

The first section of the way, thus artificially divided, leads from the conscious or potentially conscious perception of the subject matter to the point where it dives down into the unconscious mind of the psychologist. The second would then represent the unconscious assimilation of the observed material. The third stretches from the re-emergence into the conscious of the data so assimilated to the point of their description or formulation. Of the middle of these sections we can say nothing except that we have no direct access to it and that it interests us most of all. (p. 131)

It is this aspect--the nonverbal attunement to another's inner affective states--that is the focus of this study.

CULTURAL AND SCIENTIFIC BIASES THAT INHIBIT STUDY OF EMPATHIC PROCESS

Getting deeper, to the "base" of empathic capacity described by Reik (1948) is more problematic than one would expect. Western culture contains biases that may hinder the understanding and use of empathic process. Western cultures are verbal (Hall, 1976), and empathic awareness is essentially nonverbal. Western cultures are actionoriented (Condon, 1975), and empathic perception requires stilling 
ourselves to listen with our whole neural apparatus. Westerners are future oriented (Condon, 1975), and empathic process requires we not impose our purpose and ideas on another. In general, people of Western cultures want fast and efficient answers, and empathic understanding requires that we stand patient, but firm, in the face of our ignorance, fielding preemptive conceptual assumptions about the other person, knowing that we do not understand--until the reality of the other person, unencumbered by our own personal history or needs, surfaces in our experience, in its own time and with its own qualities (Reik, 1948). Likewise, biases in Western science may cripple the exploration of empathic potential. Aristotle's legacy of reductionism and the "naive reality" of Newtonian-Cartesian thought may be inappropriate to plumb the depths of Reik's (1948) concept of empathic incorporation and reverberation (defined on page 3 of this thesis). When scholars study empathy with only the tools of Western science, working definitions proliferate, moving us away from the root of empathy. For instance, communication scholars have generated an array of "types of empathy" (Appendix B). In Empathy and Counseling, a recent comprehensive examination of theory and research about empathy, Gladstein (1987) concludes:

There is a considerable body of theoretical and research literature regarding the nature of empathy . . . the empirical studies document its theorized multi-elements and multi-stages.. . Therefore, it is no longer legitimate to refer to the "nature of empathy." we must be concerned with the various kinds of empathy. (p. 187)

Gladstein (1987) describes 18 different "kinds" of empathy and urges proper testing be developed for each one. 
He adds also that "although there is considerable theoretical literature concerning empathy's development, the empirical research minimally supports the major propositions" (p. 187). Gladstein's assessment is understandable when one considers that the tools of Newtonian-Cartesian science cannot deduce the base of empathic process as defined earlier. One possible reason for this failure is that empathic events may not be repeatable: Each empathic encounter may be better viewed as new, unique, and irreversible. Empathy defined in this way cannot be studied in vivo: Experience alters us. Controlled, repeatable studies necessarily require actors who do just that: Act, rather than interact with authentic personal feeling and emotion. Further, empathy as defined here cannot be dissected, because the sum is more than the parts. Rather, empathy is seen as a dynamic system in which "the behavior of the parts can, in fact, be so unique and irregular that it bears no sign of relevance to the order of the whole system" (Weiss, 1971, cited in Capra, 1982; Reik, 1948). Consequently, even when empathy is studied as a process, the steps are necessarily arbitrary and artificially separated, giving one the false impression that it can be accessed in a linear, purposeful manner (Reik, 1948, pp. 131-132). Therefore, even though the literature posits the existence of "various kinds of empathy" (Gladstein, 1987, p. 187), it does not necessarily follow that we can learn more by studying them in isolation from each other.

Part of the problem of studying empathy lies in the fact that empathic perception is nonverbal, and therefore difficult to quantify or 
describe. This nonverbal aspect seems married to the nature of comprehension, itself. Reik (1948) comments:

It is hard to form any idea of the psychological nature of empathy, for in the controversy over the conception, the process appears sometimes as the natural, unconscious condition of psychological comprehension, sometimes as the result of a special effort and conscious endeavor. (p. 357)

Consequently, the profile of empirical studies is skewed to the later conscious stages of empathic process, the more verbal "verification stage" (Danish \& Kagan, 1971; Keefe, 1976; Reik, 1948), and "cognitive empathy" (Bachrach, 1976; Coutu, 1951; Dymond, 1949; Katz, 1963; Macarov, 1978; Mead, 1934) which requires the empathizer to use imagination or role playing to interject into the other's frame of reference. The perceptual stages which include identification and reverberation are not easily approached with customary analytical tools. This is unfortunate, because empathy's later-state efficacy may rely on genuine attunement in the core of the process.

In short, the current analytical approach does not satisfactorily plumb empathic capability. The literature takes the reader farther away from the central process, elaborating more and more about less and less. Worthwhile systematic observations, experimental results and voluminous experiential descriptions and insights have been generated, but they lack integration. In fact, Gladstein (1987) even counsels his readers to drop the central notion of the "nature of empathy" altogether (p. 188$)$. 
This thesis will attempt to move in precisely the opposite direction--toward a deeper understanding of that "nature"--using an investigative approach that may be more appropriate to the task.

1 Pribram's published and unpublished work is extensive. This thesis uses Languages of the Brain (Pribram, 1971) as a general reference because this text formalized the holographic brain concept. other works will be cited specifically where applicable.

2 Patanjali's sutras belong to antiquity. There is no mention of historical dates in the ancient Indian treatises, making their chronological placement difficult (Taimni, 1983, p.xiv). Because of this uncertainty, this thesis will not reference a date after his name. Authors of individual translations and explanations will be referenced where appropriate. 
CHAPTER II

METHOD

What guidelines, then, are to be used? How is the experience of empathy to be approached in a holistic manner? How does one systematically tease apart the inscrutable? Or give words to the wordless? Or quantify the qualitative? or describe what is intensely personal, ongoing, and diverse? Capra (1982) addresses these questions in the following statement.

Science, in my view, need not be restricted to measurements and quantitative analyses. I am prepared to call any approach to knowledge scientific that satisfies two conditions: all knowledge must be based on systematic observation, and it must be expressed in terms of selfconsistent but limited and approximate models. These requirements--the empirical basis and the process of model making--represent to me the two essential elements of the scientific method. Other aspects, such as quantification or the use of mathematics are often desirable but are not crucial. (pp 375-376)

His approach warrants further examination as an appropriate method for the study of empathic process.

\section{SYSTEMS SCIENCE AS AN ALTERNATIVE APPROACH}

Systems science is an alternative investigative approach that uses models to examine a subject in terms of process, relationships and integration.

The systems view looks at the world in terms of relationships and integration. Systems are integrated wholes whose properties cannot be reduced to those of smaller units. Instead of concentrating on basic building 
blocks or basic substances, the systems approach emphasizes basic principles of organization. (Capra, 1982, p. 266)

The term was first used by Laszlo (1972) and was taken up and developed more fully by Physicist Fritjof Capra (1982) in his book The Turning point (pp. 265-304). Capra describes it as a viable alternative to the reductionist approach for studying living organisms.

Systems science cannot replace the more orthodox reductionist method. Rather, it provides a holistic complement to reductionism that is appropriate for dynamic, rhythmic, living processes. It has the capacity for "progression from multiplicity and chaos to oneness and order" (Laszlo, 1972, cited in Capra, 1982, p. 288). These complementary approaches enhance one another: A reductionist approach to understand detailed mechanisms, and a holistic approach to understand the integration of these mechanisms into the functioning of a system. "Reductionism and holism, analysis and synthesis are complementary approaches that, used in proper balance, help us obtain a deeper knowledge of life" (Capra, 1982, pp. 267-269). Capra (1982) observes that these complementary modes are reflected in the very structure and functioning of our brains: The left hemisphere seems to specialize in analytic, linear thinking and sequential processing; and the right hemisphere seems to function predominantly in a holistic mode that is appropriate for synthesis (p. 293).

Systems science uses models and metaphors as aids for analysis and is weighted toward examination of process, synthesis, integration, and description of interrelationships to understand the nature of living processes. This approach could produce interactive keys for a deeper 
understanding of the base of empathy, while giving new insights for improving the earlier and later stages of empathic process. "Systems thinking is process thinking; form becomes associated with process, interrelation with interaction, and opposites are unified through oscillation" (Capra 1982, p. 267).

The process of empathy as described by Reik is a natural subject for this approach. Empathy may be described as a living, interactive, open process. As such, is not content oriented. Rather, as it is presented here, empathic perception involves a neurological process by which content is obtained and "in-formed." As such, it may be appropriate to examine it with the systems science perspective:

So far there have been very few attempts to apply the dynamics of self-organizing systems to neural phenomena, but those currently undertaken have brought some encouraging results. In particular, the significance of regular fluctuations in the process of perception, in the form of frequency patterns, have received considerable attention. (Capra, p. 293)

Because empathy involves perception, systems science may offer insights into the process which would be unobtainable otherwise. An expanded perspective of empathic process makes it inseparable from other aspects of human perception not always associated with empathy. For instance, the capacity for interpersonal empathy may be the the same one used for aesthetic appreciation (Dewey, 1934; Lipps, 1903, 1926), or for kinesthetic identification with moving objects (Bennett, 1972; Lipps, 1903), or for direct perception of objects themselves (Buber, 1948; Koestler, 1949, cited in Goldstein \& Michaels, 1985; Patanjali, date unknown). More subtle use of this same ability may enable the 
attunement of one's attention to perception of inner states and thoughts (Patanjali, date unknown). In other words, a systems science perspective allows us to see empathic perception as central to a wide range of perceptual processes--berhaps even basic to them.

Capra (1982) explains that the human nervous system may be considered as a system, and that it has been relatively impervious to reductionist approaches.

The organ of neural mentation--the brain and its nervous system-is a highly complex, multileveled, and multidimensional living system.. . The nervous systems of . . . humans are so complex and display such a rich variety of phenomena that any attempt to understand their functioning in purely reductionist terms seems quite hopeless. Indeed, neuroscientists have been able to map out the structure of the brain in some detail and have clarified many of its electrochemical processes, but they have remained almost completely ignorant of its integrative activities. (pp. 292-293)

However, to study human perception systems science requires that the context be enlarged beyond the brain. Capra (1982) uses the term "mind" in the same sense as Gregory Bateson who said "Mind is the essence of being alive" (p. 290). He explains that the human nervous system cannot be seen as separate from the larger matrix within which it operates, explaining "There are larger manifestations of mind of which our individual minds are only subsystems" (p. 291). Consequently a systems approach to any aspect of human perception will also necessarily incorporate the context within which mind, perception and mentation operate, requireing thatthe study transcend that of human behavior alone:

From the modern systems perspective, we can say that mentation, being the dynamics of self-organization, represents the organization of all functions and is thus a meta-function. At lower levels it will often look like behavior, which can be defined as the totality of all 
functions, and thus the behaviorist approach is often successful at these levels. But at higher levels of complexity mentation can no longer be limited to behavior as it takes on the distinctive nonspatial and nontemporal quality that we associate with mind. . . . mind is immanent not only in the body but also in the pathways and messages outside the body. p. 291.

A systems science approach would also necessarily take into

consideration the continual interplay between "the world outside" and

"the world within:"

There is a continual interplay and mutual influence between the outer world and our inner world. The patterns we perceive around us are based in a very fundamental way on the patterns within. Patterns of matter mirror patterns of mind, colored by subjective feelings and values. The modification of sensory perception by past experiences, expectations, and purposes occurs not only in the interpretation but begins at the very outset, at the "gates of perception." Numerous experiments have indicated that the registration of data by the sense organs will be different for different individuals before perception is experienced. (pp. 294-295)

Thus the models chosen for study will need to address principles of organization and interaction that include both inner neural organization and the information matrix within which it is immersed.

An equally important consideration is that systems science is consistent with new paradigm science which, among other shifts in emphasis, alters the classical view of science by including the crucial role of the observer's consciousness in the process of observation, thus challenging the idea of a totally objective description of nature.

The Cartesian notion of separate objects and our experience with cameras have led us to assume that our senses create some kind of internal picture that is a faithful reproduction of reality. But this is not how sensory perception works. Pictures of separate objects exist only in our inner world of symbols, concepts, and ideas. The reality around us is an ongoing rhythmic dance, and our senses translate some of its vibrations into frequency patterns that can be processed by the brain. (Capra 1982, p. 301) 
Systems science may thus afford communication scholars an opportunity to avoid some of the limitations of Newtonian-Cartesian science and explore empathic perception with an approach which may be more appropriate to its nature as an interactive human capacity.

In summary, for the reasons identified above, an understanding of empathy as process may not be amenable to Newtonian-Cartesian, mechanistic, linear, quantitative, inductive, and experimental methods alone. Because it is a living, communicating, open process involving human perception, the study of empathy may also benefit from systems science which uses models and experiential observation. Such an analysis will be weighted toward examination of process, synthesis, integration, description of interrelationships, and use of analogy and metaphor to understand the nature of living processes.

\section{CHOOSING APPROPRIATE MODEIS FOR EMPATHIC PROCESS}

The neurological model selected should address several questions relating to empathic process: What happens when our senses perceive something outside ourselves? How does the stimulus inform itself into our nervous system? What internal programing acts upon the information? What creates increments in awareness? How faithful is the inner representation of outer reality? What factors influence the content of awareness? What aspects of the perceptual process are under our control? To what extent is the process shared?

Until recently, no neuropsychological model could address all these questions. Although mused upon and written about for centuries 
and considered the province of the softer sciences--philosophy, psychology and metaphysics--most mind/brain questions have been conjectural until the latter part of this century. Even now, brain research reflecting the assumptions of B. F. Skinner's (1938) behaviorism or the current metaphor of cognitive psychology (the brain as a computer) fall short of these challenges. Pribram (1985b) comments :

The problem with current cognitive psychology is that its simulations are pretty well limited to intentional problemsolving behavior and to perceptual experience; it has done rather poorly with the latter. (p. 6)

He adds more specifically:

What is wrong with the current paradigm in cognitive psychology is that it is based solely on analogy with the serial processing computer and Von Neuman architecture. Serial programing is excellent for symbol manipulations but fails to provide access to the richness of texture involved in image processing. And all thought is not imageless. . - The deficiency is compounded by the view that, in the nervous system, serial architecture is represented by a Euclidian system in which single elements, single neurons serve as detectors of single feature, single percepts, single cognitions. (p. 6)

A new model or organizing principle was needed, one that could encompass the contradictions, illuminate the paradoxes and integrate the efforts in the uncoordinated world of brain research and, in addition, explain the resiliency, richness, redundancy, and creativity of human perception.

Pribram (1971), dubbed the Magellan of the Brain by the popular press for his impressive credentials as a neurosurgeon and in research design (Goleman, 1979), has assembled considerable evidence which indicates that the core process of human perception yields to the same 
mathematics used to create holograms--the Fourier Transform. Upon the strength of this discovery and intuitive insights backed by years of brain research, he constructed a holonomic model of human perception which explains many of the intransigent questions about demonstrated perceptual capacity. Pribram, who has maintained an intense interest in mind/brain issues, adds, "and, what is most important, there is room in these models for precise descriptions of processes which lead to intuitions, affects, attention and intention" (1985b, p. 6). This model is relatively new with as yet unexplored significance for our understanding of empathy. Taken together, these considerations make the holonomic brain model an interesting and promising choice for the study of empathic perception.

It is not the purpose of this thesis to support holonomic brain theory. That is a task for neuroscientists. Rather, these pages will describe the model which Pribram has defined and study its relevance to empathic perception. This study will search for organizing principles and interrelationships. The structures examined will be the structures of process. The simplicity and universality of the holonomic neurological process has far reaching implications for communication that beg to be explored. One can approach the subject with "if, then" reasoning, explaining features of the model which, if correct, would then have this or that implication for empathic communication. However, to make the study truly interesting and to give more depth to the inquiry, the thesis will add two more elements: (a) an Eastern model of human perceptual capacity--Patanjali's Yoga Sutras-- 
that includes instructions for effective use of attention; and (b) descriptive excerpts from Theodore Reik's (1948) book, Iistening With the Third Ear, which give detailed descriptions of his insights into the process, experience and use of attention in empathic perception.

The second model, Patanjali's Sutras, is a classic Eastern exposition of human perception based upon the Rg Veda. Although it is ancient and the product of another culture, Patanjali's sutras are only now beginning to be recognized in the west as a useful experiential model of perception.

Two researchers, Mishrital Jain and Kamal $M$. Jain of the Maryland Psychiatric Institute, have demonstrated how Patanjali's classic [treatise on perception] conforms to all the essential criteria of the scientific method in defining the precise methods by which a trained practitioner might experience very precise phenomenological states... . The pertinence of the reference to Yoga as science is to recall the fact that consciousness has been found to be a central phenomenon in both the natural and psychosocial sciences, and yet one that is not explainable or reducible to materialistic models. (Pelletier, 1978, p. 249)

Patanjali's work, in effect, operationalizes the perceptual potentials implicit in Pribram's holonomic model by addressing the role of attention in perception. Ten years ago a readable English translation of these Sutras was difficult to locate. Today there are several scholarly translations (Aranya 1983; Arya 1986; Ghosh 1980; Taimni, 1986).

Advantages of Using West-East Models

It is possible and certainly easier to study empathy using either the holonomic paradigm or Patanjali's Sutras. However, a unique opportunity would be missed, because the two work together 
synergistically to address the question of empathy more completely. Pribram's model contextualizes perceptual capacities, and Patanjali's model operationalizes them; Pribram's work legitimizes the model scientifically, and Patanjali's work illuminates perceptual principles experientially; Pribram enables us to map empathy neurologically, and Patanjali purports attentional methods for attuning to another person's mind and emotions with greater awareness and purpose. Both have profound implications for the basic way we interact with others and assimilate information.

Just as an object is better illuminated if the light emanates from more than one source. This study of empathic perception may benefit from combining insights from divergent perspectives. The creative aspect of the study may be enhanced by bringing them together. Here an "as if" component can be added. For example, what would be the implications for our understanding of empathic process if Pribram's description of "distributed memory" could be considered a corollary of Theodore Reik's description of the unconscious? Or, what does it mean for the conscious use of the holonomic brain model if Pribram's definition of "cognitive commodities" and internal "holophrases" has its counterpart in Patanjali's explanation of "mental modifications"? Surmounting the obstacles of sematics to discover genuine and meaningful commonalities might enhance the study because the holographic paradigm and the sutras appear to both address issues of perception while both complementing and involving each other. Each may help to clarify the other. Although the first is a product of the west and the second is a 
product of the East, they purport to speak to the same fundamental human capacities of perception. Now, with the conceptual bridge provided by Pribram's new theory, the social sciences can examine classic Eastern contributions to neuropsychology more assuredly, because holonomic theory not only recontextualizes western understanding of human perceptual capabilities, it also provides exponents--mathematical and conceptual (explained in Chapter III)--which help explain and interpret classic Eastern works on direct perception.

To the student, the invitation to use these models to explore the implications for human communication that may be waiting in the combined perspectives is irresistible and proper. To quote Robert J. Oppenheimer (1954),

The history of science is rich in the example of the fruitfulness of bringing two sets of techniques, two sets of ideas, developed in separate contexts for the pursuit of new truth, into touch with each other. (p. 96)

It would also be a presumptuous undertaking except for the fact that both models are simple at their core--only at their core--and appear to share many of the same interactive principles--such as holism, use of attention, isomorphism, and nonlinear correlations. These principles may also be central to the subjective experience of empathy.

A final advantage of using two models is that East and West biases are checked and balanced. Western science has rigorous standards for material proofs; Eastern thinking has equally rigorous requirements for experiential proofs. The West is suspicious of subjective accounts; the East is suspicious of material limitations. The west empowers instruments of measurement that take us to ever smaller particles and 
interactions; the East empowers direct perception ${ }^{1}$, and inference from direct perception, that illuminates principles of relationships and interaction.

\section{Narrowing the Focus of study Within the Models}

This undertaking would be formidable without the focus inherent in the holonomic brain metaphor. The paper will start there, with an explanation of Pribram's model as it relates to empathic perception. Using a systems approach, the question "what neurological process is at the base of perception?" will be explored. The human perceptual potentials inherent in the Fourier Transform will be explored and Pribram's clarification and redefinition of isomorphism will be explained. Interactive principles will be explored and assigned a place within the three-fold orders of "constructional reality"--the unfolded, enfolded and transforming domains--made possible by the neurological transforming capacities described by the model.

Patanjali's concise instructions on the nature, uses, and capacities human perception follow logically. After a short orientation to the work, special attention will be given to those sutras which describe specific obstructive mental states that inhibit direct perception, followed by a discussion of Sutras dealing directly with direct perception of the contents of other minds. Contemporary insights and descriptions, particularly Reik's (1948), on use of attention and perception will be incorporated where appropriate, to expand upon Patanjali's explanations. 
Finally, the last chapter recontextualizes the relationship of empathy to the field of communication and expands Reik's description of the empathic process in light of the perceptual possibilities implicit in the holonomic model.

The synthesis involved in this approach could be compared to that of a weaver. The holonomic model comprises the overall design, but the descriptions, examples and research that fill it in come from the threads of diverse and sometimes unfamiliar sources. Pribram's work is not well known to communication scholars, nor (in the west) is Patanjali's. Although Reik's (1948) outline of empathic process is used in textbooks on empathy, few authors reference his long discourse on the subtle use of attention described in Istening with the Third Ear. The exactness of Sanskrit words applied to mental processes, the holistic potentials implicit in the Fourier Transform and the holographic process itself are foreign to most as well. It is hoped that the unusual fabric derived from these threads will reveal a design of perception that underlies the experience of empathic perception.

Again, it is not the purpose of this paper to justify or prove these models, but rather to explore their possible implications for communication. This study will use the information that scientists, theorists, and scholars have already produced to help develop and color the map while searching for principles, interrelationships, reclassifications and qualitative dimensions within the models that shed light on the process we call empathy. It is all new, rich territory for the examination of human perceptual capacities, especially when combined 
and grounded with Reik's (1948) descriptive and authoritative insights on selective attention and empathy. 
CHAPTER III

HOLONOMIC PERCEPTION AND EMPATHY

THE HOLONOMIC MODEL

\section{Background}

When Karl Pribram (1971) published Languages of the Brain, he introduced a new neurological model to explain anomalies of human perception. At that time, most research projects were based upon the assumption that the brain is a biological computer organized on a localization of function principle. Each area of the gray matter, like a module or computer chip, was believed to have a specific task. Each cell was thought to function in a binary on-off fashion to store specific data in network fashion. This approach assumed the perceptual principle of geometric isomorphism. "Iso" is a Greek word for "same" and "morph" is Greek for "form." Geometric isomorphism means that every mental and motor process has a localized physical counterpart, an engram, in the gray matter within the cranium, spine and sensory organs. Other assumptions follow this accepted model. For example, to understand brain function we must probe, chart, and label areas of the brain; individual neurons are storage banks for specific memories; activity involves intricate Iinear "wiring" of nerve centers within the brain; brain damage should result in irrevocable loss of ability; mind, thoughts, and experience are private and separate from other minds; thoughts originate from neural activity in the brain; and so on. 
However, actual scientific experiments and experience provided stubborn data to the contrary, particularly with regard to the brain's residence to damage. In Pribram's (1978b) own words, "the story lof the holonomic brain model] begins, not with perception. . . but with memory" (p. 16):

Specific memories are incredibly resistant to brain damage. Removing a hunk of brain tissue or injuring one or another portion of the brain does not excise a particular memory or set of memories. The process of remembering may be disturbed in some general way, or even some aspect of a general process may be disrupted. But never is a single memory trace of some particular experience lost while all else that is memorable is retained. This fact has become well established both through clinical observation in man and through experiments on animals. Thus in some way or another memory must become distributed--the experienced input from the senses becomes spread over a sufficient expanse of brain to make memory of that experience resistant to brain damage. (p. 16)

For example, large areas of the cortex can be removed or destroyed by disease without apparent deficit in performance in experimental animals (Pietsch, 1981). Although nerve cells are known not to regenerate, individuals who have brain damage often regain lost capacities and relearn lost skills. Contrary to expectations, electrodes implanted in the brain failed to find a single engram (Lashley, cited in Pribram, 1971, p. 26). Researchers could not evoke a predictable response from specific nerve cells. Instead, several implanted electrodes revealed rhythms of activity dispersed throughout the nervous system.

Additionally, there was no significant difference in the kind of neural responses found in different areas of the brain. Synapses throughout the cerebral cortex, midbrain, limbic area, spinal cord and body all function in essentially the same manner (Pelletier, 1978, 
p. 143).

Added to these puzzles was the enigma of perceptual capacities that could not be accounted for by our ordinary understanding of brain function. Examples include: the constancy problem of how we can recognize an object regardless of the distance or perspective from which it is viewed; or why memory is distributed throughout the brain; or how motor skills can be transferred from one limb to the other; or why imitative learning is possible; or the great rapidity with which the brain takes in and sorts out desired information.

\section{The Hologram as a Solution to Anomalies of Brain Research}

In 1969, Pribram, then a researching neuropsychiatrist at stanford University (see Appendix C), recognized and welcomed the hologram as a possible unifying principle to the anomalies of brain research described earlier. He hypothesized that the brain's "deep structure" functions by using the same principle as holography, the lensless photographic process for which Hungarian Dennis Gabor (1948) received the Nobel Prize Pribram's insight was an evolution in concept that began years earlier (see Appendix D). Karl Lashley, with whom he worked, was the first to be intrigued by the possibility of distributed processing in the human nervous system.

Holism does not rest its case on the structuralist's dubious dialectical position, but on the prima facie evidence from some of the finest research ever conducted in psychology or biology--thirty years of exhaustive, imaginative and carefully controlled laboratory investigations by Karl Lashley, the founder of the entire field of physiological psychology. (Pietsch, 1981, p. 37) 
Lashley died before holographic technology could be demonstrated. It was Pribram who recognized the hologram's potential for explaining the anomalies that baffled scientists. Pribram brought together research from several laboratories, including his own work at Stanford to demonstrate that certain operations of the senses are similar to those used to create a hologram. Results of these experiments indicate that the senses operate in the frequency domain, transducing patterns of physical energy into patterns of neural energy. Before going further into holonomic brain model, however, this paper will first examine the hologram, the processes that create it, and its correspondences to human perception.

\section{The creation of a Hologram}

A hologram can be defined as an interference pattern formed as a result of coherent reference light rays encountering light scattered by an object and stored as such on a sight sensitive emulsion (Appendix E). When the coherent light is retrained through the holographic plate, a faithful three dimensional image of the original object is recreated in space. The entire procedure is lensless. The mathematical principle involved is the Fourier Transform or a variant, the Gabor Transform.

Gabor (1948) conceived the holographic process in 1947. He coined the name hologram from the words "holos" and "gramma," Greek for "the whole message" (Caulfield, 1984, p. 366). His original intent was to create better resolution for electron microphotographs.

He suggested that a coherent background wave and the waves refracted by the same [coherent light] could produce interference patterns that would store both amplitude and 
spatial phase information. Then, in a second step, these stored patterns could be used to reconstruct an image of the original tissue. (Pribram, Nuwer, \& Baron, 1974, p. 421)

However, his insightful discovery could not be demonstrated immediately. To create an efficient hologram, a coherent beam of light was necessary to "accurately" perform the Fourier Transform and produce pictures of high resolution. Then the laser was developed, and Emmet Leith and Juris Upatnicks announced successful construction of holograms in 1965 , using Gabor's lensless procedure (Caulfield, 1984, p. 366).

If one could view a hologram of a face, one would see a life-like, three-dimensional replica, sharply focused, hanging ghostlike in space. Moving to the right or left, the view would change to reveal more of the right and left sides of the face. Elevating oneself, one could see more of the top of the head.

Given the opportunity to examine the holographic plate recording the face, one would see, instead of an ordinary negative image, a surface covered with patterns of converging wave patterns like fingerprints. No sign of the original image can be found anywhere on the plate, yet when a beam of coherent light is trained through the plate, the face reappears three dimensionally in space.

If one were to smash the holographic plate with a hammer, retrieve a fragment, and illuminate only that portion, the entire image would again reappear, but this time with diminished resolution. The face would be somewhat blurred. Cut the fragment in half, and the whole can still be projected in space, but with proportionate loss of clarity. All fragments would show some aspect of the entire view, but each would vary in its emphasis. This is a peculiar and revolutionary attribute of 
holography: The whole of the picture is contained in each part of the plate. Why? Because coherent light, once disturbed by reflecting off an irregular surface, carries a record of that disturbance via repeated irregular light wave imprints to all parts of the plate. This holistic phenomena is "captured" on the holonomic plate which becomes a medium for the transforming process. It is this processing medium which provides the holistic metaphor for perception and memory.

The peculiarity of holograms is that because information becomes stored throughout, each part of the hologram encodes the whole. The relationship between whole and part is therefore both distributed and enfolded. In fact, the holographic representation enfolds the coordinates of space and time as well as the specific images of objects that are extended in space and time. Thus the memory store is organized neither in space (in any place in the brain) nor in time (in a sequence as if it were registered on a tape), though spatial and temporal markers may well accompany any specific to-be-remembered episode. The feasibility of rapid and extensive correlations stems from the distributed nature of the transformed store. (Pribram, 1983a, p. 36)

Therefore, each point of the original image is distributed throughout the hologram. Conversely, each part of the hologram contains information from the entire original image and can therefore reproduce it. The repeated superimposition of essences supply innumerable correlations from which the details, the particularities of the object emerge three dimensionally and projected out from the plate when the total hologram is illuminated. The process provides for rich and resiliant imaging forming properties akin to that of human perception (Pribram, 1971, p. 166).

The holistic principle illustrated above is inherent in holonomic ${ }^{2}$ model. Here holism is qualified in that parts of the plate differ in density of interacting wave forms; the plate is not uniform. However, 
it is also radical and inclusive in that it lacks specific boundaries. Recognizing this, Pribram (1979a) makes this useful distinction,

The holistic principle of the hologram is different from the Gestalt principle that "the whole is greater than and different from the sum of its parts." It carries wholism much farther. "The whole is contained or enfolded in its parts" and the very notion of parts is altered because parts of a hologram have no specific boundaries. (p 25-6)

It is important to differentiate between the following aspects of the holographic process. Each aspect of the holographic process involves the other, but with differences in emphasis:

1. The holistic and correlative phenomena of distributed wave forms. This phenomena takes the form of scattered, interacting light waves which carry specific information throughout the whole, creating correlations. It is ongoing and changing.

2. The empirical transforming process that encodes and decodes the scattered light. This process is unique in that both devolves and covolves, reversing the effect from distributed light information to form, or form to distributed information, thus making possible the reconstruction and location of the original light event.

3. The media that collects and records them. The collecting device is the holographic plate that imprints the distributed information in the form of intersecting wave patterns that can be decoded later. This collecting device retains both the holistic potential of the intersecting wave forms and the transforming potential of the Fourier theorem.

Discrimination between these three aspects of holography will help bridge distinctions between the hologram and the holonomic model of 
perception which describes the core of empathic process. It will also be useful in the analysis of principles of perception and interaction included in the model.

However, first it is necessary to examine the Fourier Theorem (Appendix F). What is the structure of the process? How is the process carried out? The Fourier Transform warrants closer examination because it provides the empirical support for the contention that the hologram is a literal metaphor for human perception.

\section{The Fourier Transform}

The Fourier Theorem states that:

any pattern of organization can be analyzed into, and represented by, a series of regular wave forms of different amplitudes and frequencies. These regular wave forms can in turn be superimposed, covolved, with one another by way of the inverse Fourier Transform to obtain the original space time configuration. (Pribram, 1984b, p. 21)

The mathematics which explains the process is a form of calculus first developed by Gottfried Wilhelm von Leibniz in 1714 (Brain/Mind Bulletin, 1979, n.p.). It is used to break up a compound wave into its constituent components and, conversely, to generate a compound wave from its basic components. It can be applied to any and all wave forms and frequencies. It is one of the most far-reaching principles of mathematics in physics because: "Any physical property--density, pressure, velocity--which varies periodically with time and whose magnitude or intensity can be measured, may be represented by Fourier's series" (Mellor, 1955, cited in Pietsch, 1981, p. 127). Technology harnesses the Eourier Transform for a multitude of functions requiring 
imaging and location of objects in space. Among these, the fast Fourier Transform is responsible for CT (computerized tomography) scans and magnetic imaging.

Simulations of image processing by computer have found no technique other than the holographic to provide the rich texture of scenes such as those that compose our experiences. And the complicated computations that go into three dimensional $x-r a y$ imaging by computerized tomography have relied heavily on the fact that such computations (mostly correlations) are performed readily in the frequency (holographic) domain. (Pribram, 1978b, p. 17)

Its unique attribute is that the identical equation covolves and devolves itself. The procedure is slow and arduous when done with a pencil, but yields to computer technology with ease.

Holographic photography performs an automatic Fourier Transform with coherent light. When the disturbed beam from the subject reaches the plate, it is met from an angle by an undisturbed coherent beam and, like ripples merging in a pond, the merging light rays form an interference pattern which encodes the image of the subject throughout the plate:

Each point of light diffracted from the object becomes blurred and is spread over the entire surface of the film (the equations that describe this are called spread functions), as is each neighboring point of light. The spread is not haphazard, however, as the blur would lead one to believe. Rather, ripples of waves move out from the point of light much as ripples of waves are formed when a pebble strikes the smooth surface of a pond of water. Throw a handful of pebbles or sand into the pond, and the ripples produced by each pebble or grain will crisscross with those produced by other pebbles or grains, setting up patterns of interfering wave fronts. The smooth mirror-like surface has become blurred, but the blur has hidden within it an unsuspectedly orderly pattern. If the pond could suddenly be frozen at this moment, its surface would be a hologram. The photographic hologram is such a frozen record of interference patterns. (Pribram, 1978b, p. 16) 
In a sense, each portion of the subject is omnipresent on the plate "in code," awaiting retrieval to its former configuration which happens automatically when the reference beam is again trained through the plate. This beam subtracts out the interference pattern and devolves the encoded vibratory pattern to the original light configurations that one can recognize as the original subject. Again, the image suspends, life-like, in space. This is a unique feature of the Fourier Transform; the same transform both encodes and decodes, enfolding information into a distributed domain independent of three-dimensional space and time, then reversing the process to "re-member" the original configurations. It is empowering in the sense that it holds potential for creative control of the process.

\section{Analogies Between the Holography and Perception}

Dr. Karl Pribram's (1971) theory of brain processes explains that the human nervous system is equipped to process vibratory information in a manner similar to the hologram. Wave forms are registered on the senses and are processed in the nervous system in the infinitesimal spaces between the dendrites of the neurons. Taken en masse, these small spaces provide the computational space for the Fourier transform, or a variant, to enfold and unfold patterned information.

The holonomic metaphor may be termed a literal one because the mathematical principle used in the hologram is the same used by the senses. Our senses, like holographic equipment, register interacting wave forms, and through a neurological process that can be interpreted by the Fourier Transform (or a variant) they are able to both encode the 
interference patterns, converting them into memory, and also reproject out a representation of the original vibratory stimulus. Because of the nature of the transform and the capacity of our nervous system to decode and encode vibration, we are able to convert sensory information into a visible, tactile, noisy and moving world, and also to experience time and three-dimensional space. In other words, our brain structures see, hear, taste, smell, touch, project impressions out away from our bodies and focus them in space, and experience motion by sophisticated mathematical analysis of temporal and/or spatial frequencies, the same process used to create holograms.

We have no way of knowing the qualitative difference between what is originally registered on our senses and what is projected out again-how faithful the representation. Pribram's theory leaves room for the possibility that our senses are creators with their own limited representation. Perhaps they necessarily select out and step down impressions, just as the hologram lacks many of the qualities we perceive of the original. Nevertheless, the neurological processes here, which are assumed to be shared universally, are more sophisticated than formerly assumed.

"Sophisticated" must not be confused with "complex." Holography utilizes an elegantly simple physical system. This structure, and its many variations, operationalizes the Fourier Transform, or a variant, to encode and decode light with precision and efficiency. Mathematics gives language to the process, but because of the physical construction 
it employs, holography--and the human nervous system--performs the transforms automatically.

If Pribram's theory is correct, the encoding process creates an added component to perception, making possible access to a pure transform domain that is collapsed, distributed, and independent of space and time. This distributed order may provide new explanations for the study of the core processes of empathic perception.

In addition to the correspondences between holography and human perception explained earlier, other interesting similarities can be demonstrated. Today, with a technology using various colored gases, a broad spectrum of coherent light rays can be produced. With this, more unique holographic capacities that model human perception became evident. For example, holographic technology enables us to layer many pictures on one plate by using different frequencies of coherent light for each exposure. Each separate reference beam will elicit its own picture from the same plate when refocused through it. In addition to altering the frequency of the reference beam for new images, the altered angle of the laser can also create different images in the same space.

A holographic plate does not simply store an image-code. By altering the angle at which the light from the object strikes the plate and by altering the frequency of the laser beam, one cubic centimeter of plate can store ten billion codes in its finely layered texture. When a laser light of the same frequency and angle re-illumines the plate the original image is recreated, like a memory relived by reproducing its context. (Hampden-Turner, 1981, p. 96)

Thus the holographic process demonstrates flexibility and redundancy in process to create vast quantities of information to be stored in a very limited space. "The entire contents of the Library of Congress can 
currently be stored on holofischs (microfilm recorded in holographic form), taking up no more space than is contained in an attache case" (Pribram, 1980d, p. 31). Taken together with the distributed nature of the brain, this capability is of special significance to our understanding of the resilience and richness of memory and perception.

Holographic storage is the most sophisticated, the most economic, and, from an evolutionary standpoint, the "fittest-to-survive" of any method known to man. A computer in comparison is disabled by the malfunctioning of a single circuit. (Hampden-Turner, 1981, p. 97)

Holograms mimic human experience in yet another way. Coherent reference beams can be replaced by specific wave pattern forms. This means two pictures--or sensory experiences--can encode and decode each other. For example, if one person's picture is merged with another person's picture to create an interference pattern on the holographic plate, the first face will appear in space when the second is refocused through the plate--and vice versa. Illumination of the stored hologram by light reflected from only one object will reconstruct a ghost image of the missing object. The living, neurological counterpart to this holographic possibility is associative memory. Thus one object acts as a reference for another "re-membered" object. Pribram adds that "this coupling also provides a powerful technique for correlating--cross correlations and auto correlations are accomplished almost instantly" $(1979 a$, p. 26).

Paul Peitsch (1981), Professor of Anatomy at Indiana University, compares the behavior of multiple holograms to the human experience of recalling, forgetting, thinking, and imagination: 
Just as important, multiple holograms mimic the actual recalling and forgetting processes: tilt the film in the reconstruction beam, and instantly, off goes one scene and on comes the next. A few years ago, I met a young man named John Kilpatrick who suggested that a person trying to recollect something may be searching for the equivalent of the correct reconstruction angle... . And suppose we pass the beams through the multiple hologram at different angles. We may, in this manner, synthesize a composite scene. And the objects in the composite scene may never have been together in objective reality. When the human mind synthesizes memories into unprecedented subjective scenes, we apply terms such as thinking, reasoning, imagining, and even hallucinating. In other words, built right into the hologramic model are analogues of much human mental activity. (p. 71)

Peitsch (1981) also observes the esoteric effects achieved with the combination of lenses and holography. If a holographer uses a lens during construction of the hologram, that identical lens must be used for reconstruction. Thus, the holographic result can be hidden or revealed by an intervening factor--an individual lens:

Holography does not require the use of lenses. But lenses may be employed to produce special effects. Leith and Upatnicks showed in one of their earliest experiments that when the holographer uses a lens during construction, he must use an identical lens for reconstruction. This fact should (and probably does) interest spies. For not even Gabor or Leith and Upatnicks could read the holographic message directly. It is a code in the most cloak-and-dagger sense of the word. A hologram must be decoded by the appropriate reconstruction beam, under specific conditions. And a lens with an unusual crack in it would create an uncrackable code for all those who do not possess that same cracked lens. (pp. 21-22)

Continuing the analogy to the mind, if the lens-like, focusing qualities of individual attention were to be substituted for the physical lens described above, then the same scene or event would be experienced differently by different human beings. Subjective human experience, difficult to communicate, could be attributed in part to attentional styles, variances in quality of attention, or differences in belief 
constructs that cause us to use attention in varying ways. For example, attention can be focused narrowly on detail, or expanded to sense the larger picture. It may attend to pure sensory information or mix this with inner holographic memories of previous experiences. It could take in lucid and ongoing impressions, or hazy disjointed ones, and so on. Like a lens, attention can magnify one impression to the exclusion of others. Like a lens, attention may be clear and coherent or cloudy and distorted. The subject of attention as it relates to holonomic perception and empathy will be developed more fully in Chapter IV.

In a paper entitled "Localization and Distribution of Function in the Brain," Pribram (1982a) summarizes several properties of holograms that parallel human holonomic perceptual processes:

(1) The holographic store [the sum of interference patterns] is distributed; (2) vast amounts of storage can be concentrated in a small holographic space; (3) image reconstruction is three dimensional, displaying constancies and parallax, and is highly textured; (4) images do not appear coextensive with the holographic store; they are projected away from the film surface; (5) the hologram has associative properties; when it is made by the reflected light of two objects, subsequent illumination of a stored hologram by light reflected from only one object will reconstruct a ghost image of the missing object.

These properties of holograms are so similar to the elusive properties that Lashley sought in brain tissue to explain perceptual imaging and engram encodings that the holographic process must be seriously considered as an explanatory device. (p. 276)

The ghostly holographic image itself, projected away from the holographic plate and hovering in space, suggests a process more intimately familiar and fundamental than mere photographic wizardry, lending it intuitive credence as a literal metaphor for the holonomic brain process. The hologram, however, is only a metaphor. "It must 
constantly be remembered that it is the mathematics of holography and brain function that needs to be compared and tested, not the optical holograms or computer instantiations of holography" (Pribram, 1982a, p. 276).

\section{HOLONOMIC PERCEPTION}

Like holographic photographic equipment, the brain and nervous system may also be structurally designed to perform transforms automatically, rapidly, and efficiently. There are, however, important differences between ordinary photographic holograms and the human nervous system. Pribram (1984c) explains:

Ordinary holograms are composed by a global Fourier transform which distributes the information contained in a spacetime image throughout the transform domain. In the visual nervous system, the distribution is limited anatomically to the input channeled to a particular cortical cell. There are, however, holographic techniques that use similar "patch" or multiplex constructions. Bracewell (1965) at Stanford University pioneered these techniques in radioastronomy by stripping together the holographic transformations of limited sectors of the heavens as viewed by radiotelescope. When the inverse transform is applied, spacetime images of the whole composite can be viewed in three dimensions.

. . This is another way of stating that the transformation is not global, and gives mathematical precision to the limits involved. . . . [in addition] the gross grain of the visual filter determines spacetime coordinates, while its fine grain describes the Fourier components. . . What is the advantage gained by this fine grain holographic-like organization? In the transform domain correlations among patterns are readily performed. (n.p.)

Pribram now uses the term "holonomic" to describe brain processes, because this term incorporates the concept of movement and may help eliminate oversimplistic assumptions about brain processes that are may be inferred from the hologram (Pribram, 1989b). A hologram is static 
and more global than holonomic processes which utilize patch holograms to create the sensation of movement and the whole. "Patch holograms are a much more powerful way of encoding than a simple hologram" (Goleman, 1979, p. 80). However, the hologram still retains valid correspondences for analyzing and demonstrating the holonomic model and will be referred to throughout this thesis.

\section{Patterned Synapses}

In the holonomic mind, the equivalent of interference patterns on the holographic plate can be found in the interacting, patterned, electrical releases in synaptic spaces:

Essentially, the theory reads that the brain at one state of processing performs its analyses in the frequency domain. This is accomplished at the junctions between neurons not within neurons. Thus graded local waxings and waning of neural potentials (waves) rather than nerve impulses are responsible. Nerve impulses are generated within neurons and are used to propagate the signals that constitute information over long distances via long nerve fibers. Graded local potential changes, waves, are constituted at the ends of these nerve fibers where they adjoin shorter branches that form a feltwork of interconnections among neurons. Some neurons, now called local circuit neurons, have no long fibers and display no nerve impulses. They function in the graded wave mode primarily and are especially responsible for horizontal connectivities in sheets of neural tissue, connectivities in which holographic-like interference patterns can become constructed. (Pribram, 1978b, pp. 16-17)

Each of these infinitesimal junctions exists between the undulating ends of two opposing dendrites, the tendril-like fingers that emanate out from the axon of each nerve (Eigures 1, 2). They are minute, making them sensitive to electro-chemical effects. 


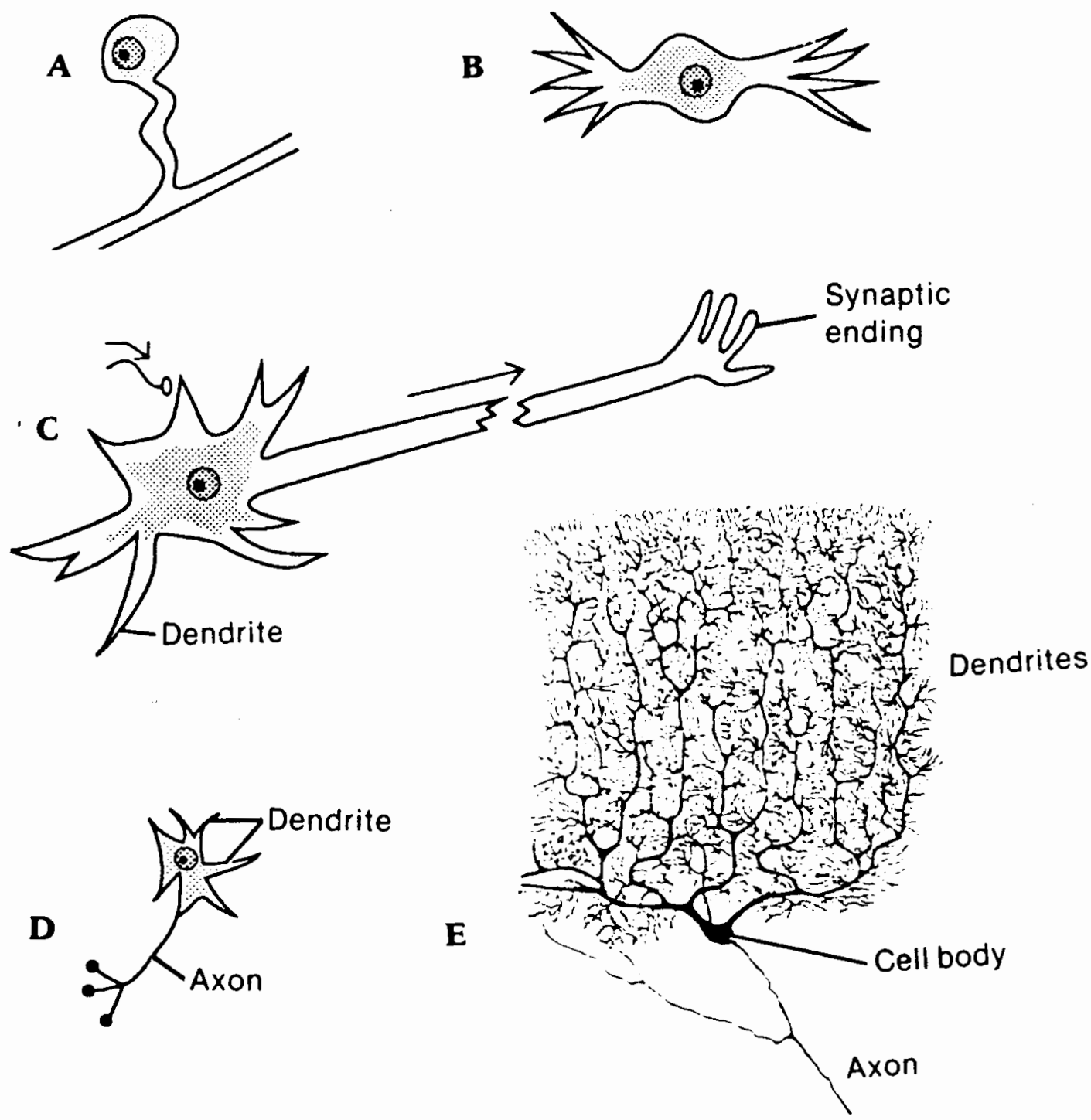

Eigure 1. Representative neurons. Drawings depicting:

(a) :Unipolar neuron like those in the sensory ganglia and cranial nerves; (b) bipolar neuron like those in the retina; (c) multipolar neuron like motor neurons in the spine, with a synaptic ending into one of its dendrites; (d) multipolar neuron with a short axon like granule cells of the cerebral cortex; and, (e) human cerebeller Purkinje cell. Source: Cajal, R., cited in Nolte, 1988, p. 2. 


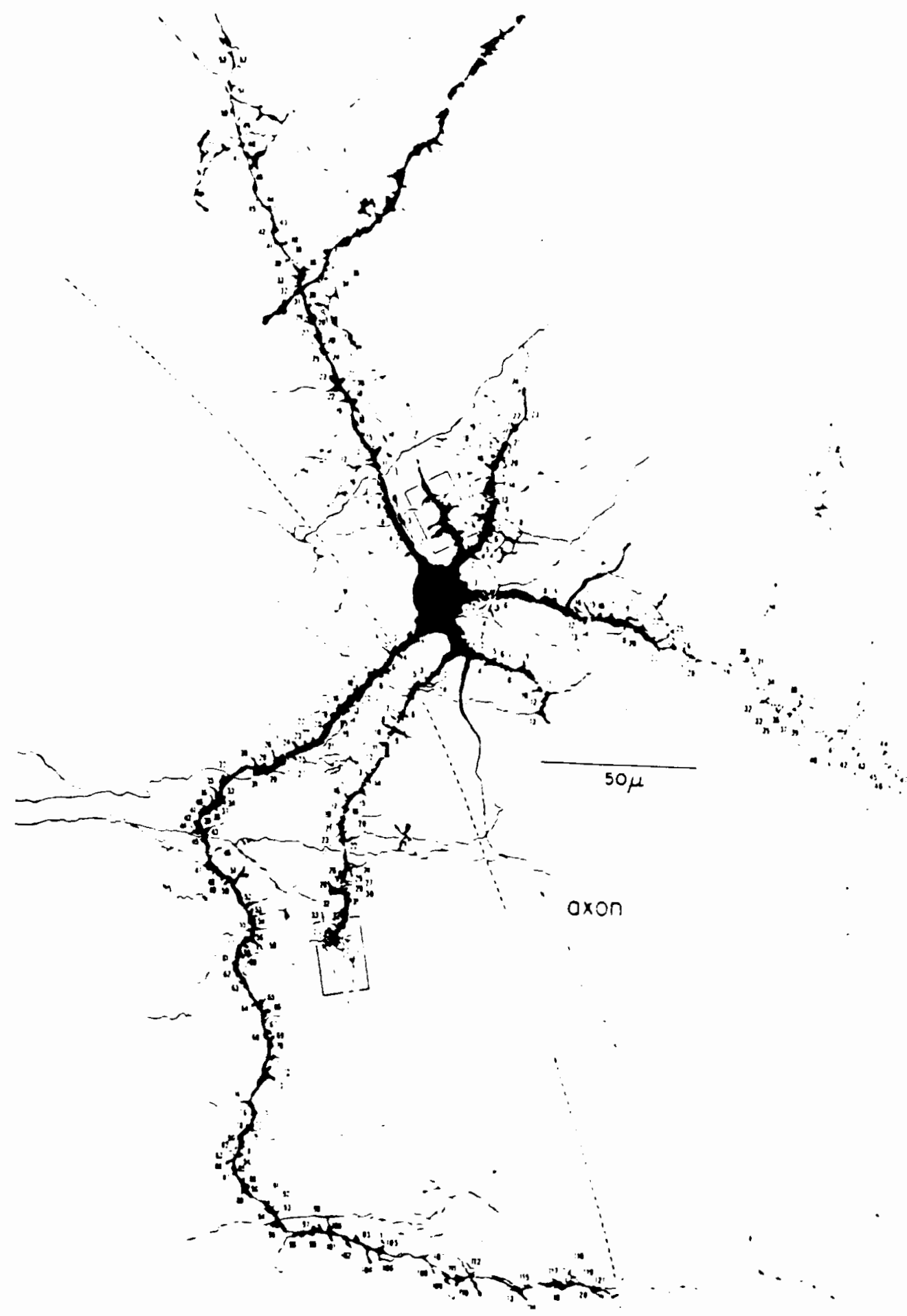

Eigure 2. Neuron and dendrites. Highly developed dendritic system of a neuron showing 121 spines. Each of these can act as a contact point with other dendrites. Source: Calvin, cited in Pribram, 1971, p. 33. 
Quite importantly, the synaptic cleft is on the order of 200 to $300 \mathrm{~A}$ or Angstroms (one Angstrom $=1 / 100,000,000$ of a centimeter), which is a magnitude in the range considered by quantum physics. In the current understanding, the transmission of nerve impulses across this cleft is initiated by a nerve impulse arriving at the end foot and causing the release of "packets" of chemical neurotransmitters, which are infinitesimally smaller than $200 \mathrm{~A}$, from the synaptic vesicles or sacs. (Pelletier, 1978 , pp. 133-134)

Billions of these tiny synaptic clefts are arranged in staged networks. The stages enable existing inner neural patterns--stored in the form of mean ranges--to horizontally intersect and modify input. These computation stages exist throughout the brain, spine and sensory organs, providing a vast spatial arena for ongoing, patterned, electrical storms that encode and decode vibratory activity. This synaptic theater can be visualized from different conceptual vantage points. Up close, it seethes with explosive activity--alive with seeming random release of electrical potentials. Farther removed, it dances with variations of rhythmic, periodic patterns. Still more removed, the combined activity takes on the predictability of standing waveforms which are recorded by the body.

The nervous system appears to give priority to the ongoing, pulsating, rapid-fire designs in this neural arena, processing the patterned vibratory activity into meaningful human experience. Although the number of neurons do not increase throughout one's lifetime, the number and configurations of dendrites undergo change (Figure 3); and although the neurons vary greatly in size and 


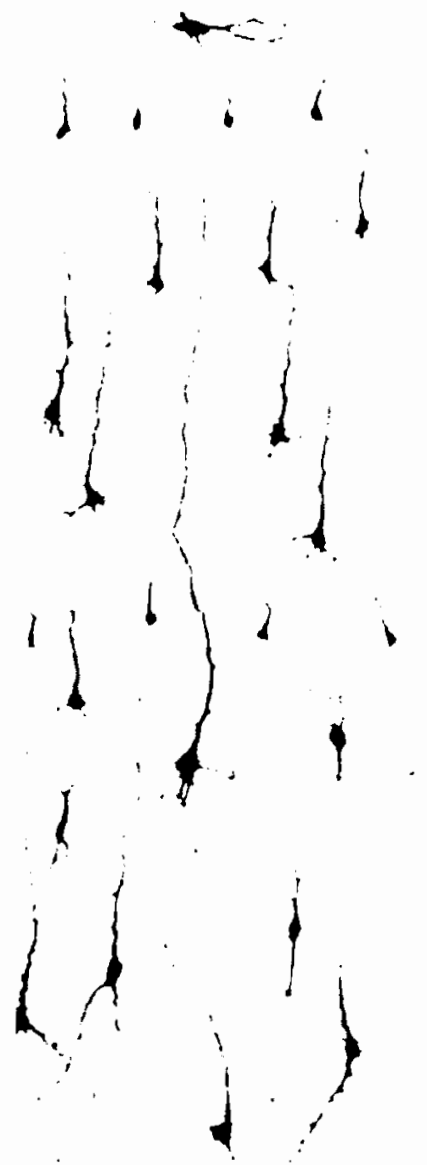

1 month

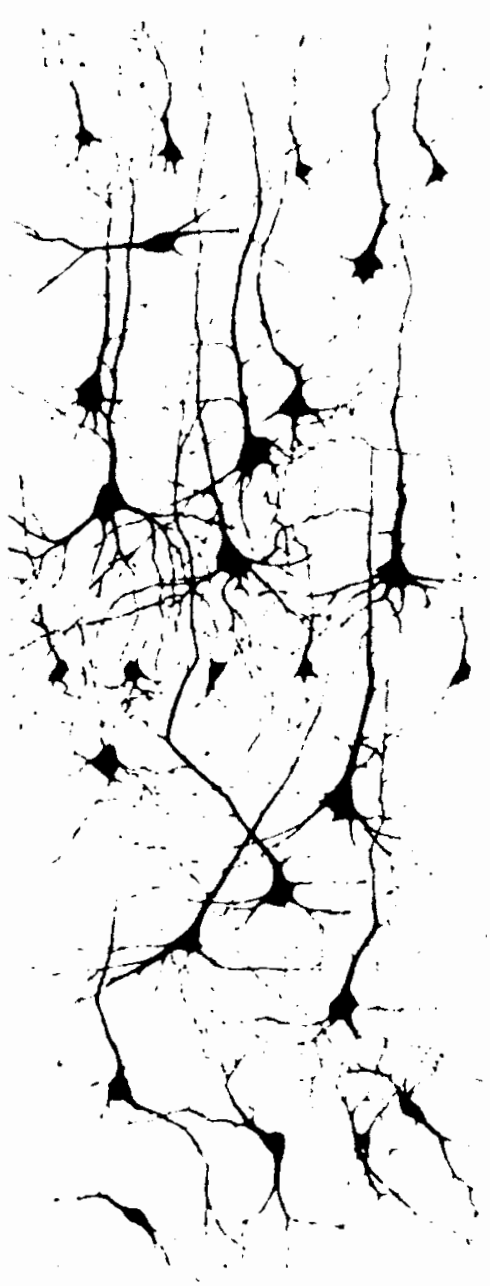

6 months

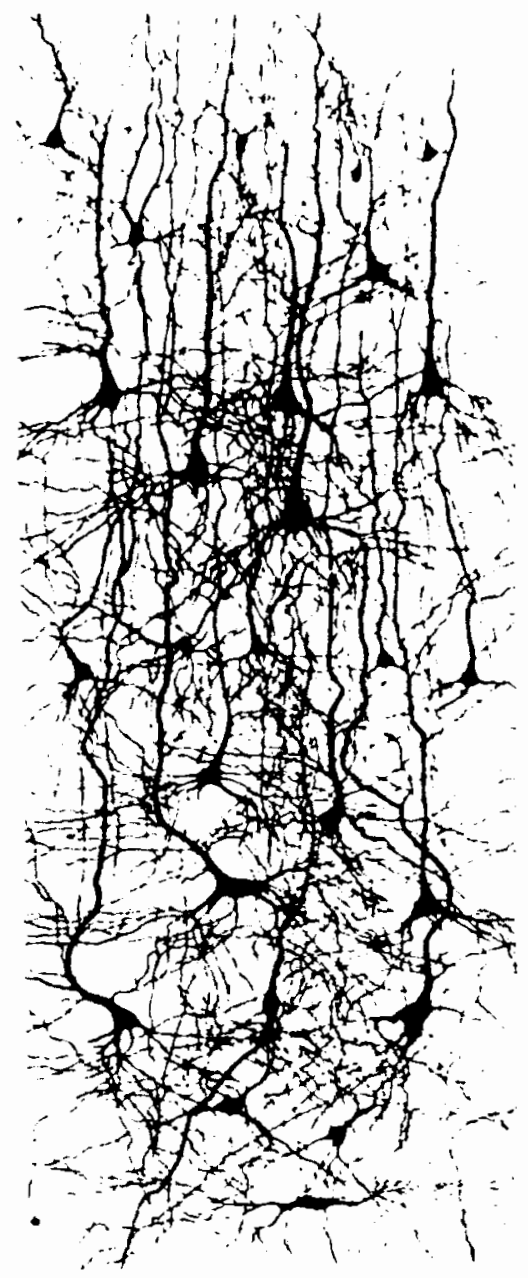

2 years

Eigure 3. Arborization and thickening of dendrites in children 1 month, 6 months, and 2 years of age. Golgistained sections of the human cerebral cortex taken from equivalent areas of the anterior portion of the middle frontal gyrus at different ages. Although the packing density of cortical neurons does not appear to increase, there is a tremendous increase in the complexity of dendritic arborizations with age. Source: Conel, J. L., cited in Nolte, 1988, p. 339. 
The basic nature of the synapse is constant throughout the nervous system. In fact, all synapses are alike in their essential features and in their mode of chemical

transmission. There appears to be no essential differences between the parts of the nervous system that, like the spinal column, are associated with autonomic activity and those that, like the cortex, are associated with mentation, imagery, and other such "higher order" phenomena of consciousness." (Pelletier, 1978, p. 134)

The conceptual shift from emphasis on the neurons themselves to emphasis upon patterned processes between the dendrites of the neurons is a fundamental first step toward understanding holonomic brain theory.

\section{Slow Wave Potentials}

More recent experimentation revealed refining information about slow-wave potentials which continuously wax and wane. When the nerve cell is probed, rhythmic alternations can be detected in the cell, even in the absence of propagated nerve impulses. These result in slow-wave potentials, which are small, short, wave-length, slow impulses of electrical activity occurring between the synapses. Thus there are two activities contributing to interfering wave patterns:

(1) nerve impulse unit discharges occurring in a binary "off-on" fashion on an intracellular basis; and (2) graded, slow-potential changes that wax and wane continuously at junctions between the neurons. This second attribute of the nervous system contributes to a key concept in Pribram's theorizing, for these continuously undulating, slow-wave potentials can be influenced by infinitesimal amounts of energy. They thus provide a model by means of which we can conceptualize how the subtle phenomena of consciousness may interact with these comparable subtle properties of the brain. (Pelletier, 1978, p. 124)

Theoretically these slow-wave potentials could provide continuous interacting wave fronts necessary for the interference patterns to encode and decode outer vibratory information of varying frequencies 
(similar to the role of the reference beam in holography). They may also be sensitive to inner states which are affected by our awareness and state of being. Subtle chemical changes in the brain may also alter the slow-wave potentials, thereby enhancing, stabilizing, diminishing, narrowing or globalizing effects produced by changing interference patterns.

In other words, hologram theory provides a model for understanding how drugs or spontaneous biochemical imbalances affect behavior: By modifying, however slightly, the brain's chemical medium they alter the ongoing activity of slow-potential waves. . . . Furthermore, these slowpotential waves may prove to be a link between centralnervous-system properties and meditation and biofeedback, since both meditation practice and biofeedback training have been associated with sustained slow-frequency brain-wave activity in ranges comparable to the range of the slowpotential wave found at neuronal junctures. Doubtless this sustained and coherent low-frequency activity of the brain profoundly affects nerve transmission, and perhaps these frequencies are intimately involved with the ensuing states of consciousness.

All of this must remain highly speculative, but there is considerable research evidence in support of these observations. (Pelletier, 1978, pp. 125-126)

\section{Ce-operativity}

Pribram (1971) reports "co-operativity" in the synaptic arena.

One interpretation of his long and very exacting explanations of cooperativity is that neurons, axons and dendrites receive, store and release their graded potentials for synaptic activity in ways that suggest the primacy of the patterned firing rather than the accumulation of electric potentials in individual neurons. This lends strong support to the holonomic model. A summary of the actual computation process is as follows: 
Nerve impulses arriving at junctions generate dendritic microprocesses. The design of these microprocesses interacts with that which is already present by virtue of the spontaneous activity of the nervous system and its previous "experience." The interaction is modulated by inhibitory processes and the whole procedure accounts for the computational power of the brain. The dendritic microprocesses act as a "cross-correlation" device to produce new figures from which the patterns of departure of axonic nerve impulses are initiated. The rapidly paced changes in awareness could well reflect the duration of the correlation process. (Pribram, 1989a, Lecture 1, p. 23) ${ }^{2}$

Even neurons without axons contribute to this synaptic array. An

example:

The ubiquity of such axonless local circuit neurons indicates that computation is strongly influenced by dendritic-dendritic interactions which modify the postaxoral dendritic processes. Perceptual processing depends therefore on network properties that extend beyond the purview of the dendrites of a single neuron. It is the synaptic event, rather than the neuron per se, that serves as the computation element. (Pribram, 1989a, Lecture 1, p. 27)

Evidence of patterns of polarization supports the theory of distributed processing :

The sub and superneuronal aspect of the dendritic microprocess, its potential to expend beyond the single neuron, provides explanatory power for both older and recently accumulating evidence that brain processes resulting in perception are distributed. . . . In a distributed process, perceptual events are represented not by single neurons but by patterns of pelarization across ensembles of neurons. (Pribram, 1989a, Lecture 1, p. 27)

These laboratory observations all lend strong support to the holonomic brain theory. More than this, however, they change a fundamental assumption about perception. Instead of thinking of the brain as constructing information from the input of a sensory nerve, we can instead think of dendritic space as the "gravitational" center of the nervous system which resonates to information. 
Memory

Memory exists throughout the nervous system in distributed form.

"The structure is hidden because it becomes enfolded and spread, distributed, into a form which displays "nonlocality" (1989b, Lecture 2, p. 9). Just as the standing wave forms are recorded in distributed form in an emulsion throughout the holographic plate thus preserving them until they can be transformed back into three dimensions through an inverse process, the neurological structures also have the capacity for recording and "re-membering" patterned information.

The holonomic brain theory contends that memory is distributed throughout the nervous system, although the retrieval mechanisms are more hard wired. The microstructure of each cortical cell is separately involved in transforming input just as each string of the sounding board of the piano is separately involved in resonating to the action of the keyboard. Because the mind enfolds memory holonomically, by way of a Fourier-like transform, the microstructure of matter can be shown to partake of the same structural properties as the microstructure of memory. (Pribram, 1983a, pp. 19-201

The recording process itself is not yet understood. Pribram postulates that hydrogen ions, changing position, are sufficiently sensitive and abundant and may be responsible (Pribram, 1989b, p. 11). In a speech sponsored by The Association for Humanistic Psychology, he suggested the following possibility:

This distributed input must then, in some form, perhaps as changes in the conformation of proteins at the membrane surfaces, become encoded into distributed memory traces. The protein molecules would serve the neural hologram in the same way as oxidized silver grains serve the photographic hologram. (1978b, p. 17)

Although the chemical component of the recording of memory has not yet been adequately subjected to research and remains speculative, the 
distributed and correlative nature of holonomic memory appears to be established,

Distributed memory correlates with and acts upon the incoming array of patterned sensory information, altering perceived experience. Pribram (1989) explains how in this summary of top-down influences on the visual system.

Within the class of systems involved in figural perception, those involved in imaging can be distinguished from those involved in the perception of object-forms. But, as in perceiving, the systems responsible for extracting the invariances (constancies) that characterize object forms interpenetrate in a top-down, corticofugal fashion, the systems responsible for imaging. This top-down interpenetration is found again when systems responsible for stimulus sampling and categorizing are considered, and one more when the systems concerned with relevance and with inference are studied. The fact that each level of processing entails both feedforward and feedback operations accounts for the paradox of the separable yet unitary nature of the perceptual experience. (1989a, Lecture 1, p. 8)

As the organization of the nervous system progresses toward the forebrain, holonomic processing is more global and boundary formation is more arbitrary and intentional (Pribram \& Carlton, 1986).

\section{Emations}

Pribram (1981, 1983a). has also formulated a four-fold schematic for the organization of emotional and motivational feelings and expressions. He calls these labile-stabile, epicritic-protocritic, affective-effective, and aesthetic-ethical. It is a macro-model of the brain which maps areas that addresses emotions, visualization, and intentionality and internalizing which pulls us toward or away from interaction with our environment (Pribram, 1981). The limbic area is 
believed to have a deciding role in the arousal of interest and attention, interacting with the frontal lobes and contributing to the globalizing of perception (Pribram, 1984a). Importantly, "envisioning and inferring engages the activities of the frontolimbic forebrain" (1989a, lecture 9, p. 28). Pribram (1983, 1989b) hopes the overall schematic will encourage co-operative research between biochemists and biophysics. More research is necessary to refine the larger processes. ${ }^{3}$

\section{Summary}

Evidence strongly substantiates the theory that neural microprocesses are governed by the same mathematical principles that make holography possible. Pribram is confident that throughout the nervous system patterned synaptic interacts provide the computational power that creates and recreates the distributed store of human experience, thus making perception, memory, thinking, and learning possible.

The discovery of the holonomic organization of memory has important consequences. As noted earlier, the brain has mechanisms for organizing and retrieval from the distributed store, and we might properly call the operations of these gnostic mechanisms "thinking." According to the holonomic hypothesis, the operations involved in thinking must invert the distributed memory into images-not necessarily visual images but also kinesthetic/tactile, olfactory/gustatory, and auditory/linquistic. An experiment by wallach and Averbach in 1955 showed that thinking had to be carried out in one or another sensory mode, that there is no such process as "pure" nonmodal thought. (Pribram, 1983a, pp. 36-37)

These pages contain an abbreviated description of holographic process and Pribram's holonomic theory. They are an attempt to summarize essential properties of the hologram and equivalent processes in the brain, then go beyond this to with this as a foundation, the 
pages that follow will explore basic principles and interactions of holonomic perception that have implications for human empathic perception. Of interest here are descriptions of process and interaction that may explain the base of empathic perception.

\section{APPLICATION OF PRINCIPLES OF HOLONOMIC PERCEPTION TO EMPATHIC PROCESS}

The purpose of this section is to ascertain interactive principles inherent in the holonomic model of perception that may apply to empathic perception. Here the term principle is defined as an essential element, a constituent or quality, especially one that produces a special effect. In addition to the holonomic principles introduced in the previous section--i.e., each part containing the whole part, vast storage potential, enfolding information apart from space and time, convertibility, and the primacy of patterned activity in synaptic space-the hologram appears to involve additional working principles which qualify the nature of human perception and empathy. Explanation of these principles is facilitated by the distinctions drawn earlier between the three aspects of holographic process, except that here the distinctions are those that apply to holonomic brain processes.

The principles of holonomic perception defined here are organized according to these three aspects--the ongoing coding process, the uncoded representation of interactive patterns that are registered on the senses, or the encoded patterns that are stored in distributed form throughout the nervous system as memory. The holonomic model is better 
comprehended when these three are considered separately. Together, however, they constitute an interactive whole.

This section of the thesis is purely exploratory in nature. The information presented here is found in the literature, but not organized as it is in the following pages. The approach here is to place holonomic perception in a larger "mental" context (see systems science approach in Chapter II) so that interactions and interrelationships can be inferred. Pribram (1976, 1979b, 1982b, 1986) calls this context "constructional realism," and alternatively "structural realism" because it includes a transforming principle which provides a reciprocal relationship between the frequency domain and the image/object domain. This tranform principle makes perception approachable with the tools of mathematics. In constructional realism "the basic function of the brain is to generate the codes by which information becomes communicated" (Pribram, 1976, p. 301). He notes that constructional realism does not deny the ordinary duality of subject/object, but subsumes it by providing a larger context for human perception (1986, p. 518). He adds, "I am inclined to accept this structuralist approach to the Mind/Brain problem because it can subsume the others and bring to bear additional scientific evidence" (1982b, p. 251).

The objective here is to learn more about the subsystem--holonomic perception--by examining its interaction within the larger system-constructional realism (see figure 4). First, however, the three possible aspects of constructional realism will be more fully elaborated. 
CONSTRUCTIONAL REALISM:

A SCHEMATIC DEPICTING POSSIBLE PERCEPTUAL INTERACTIONS
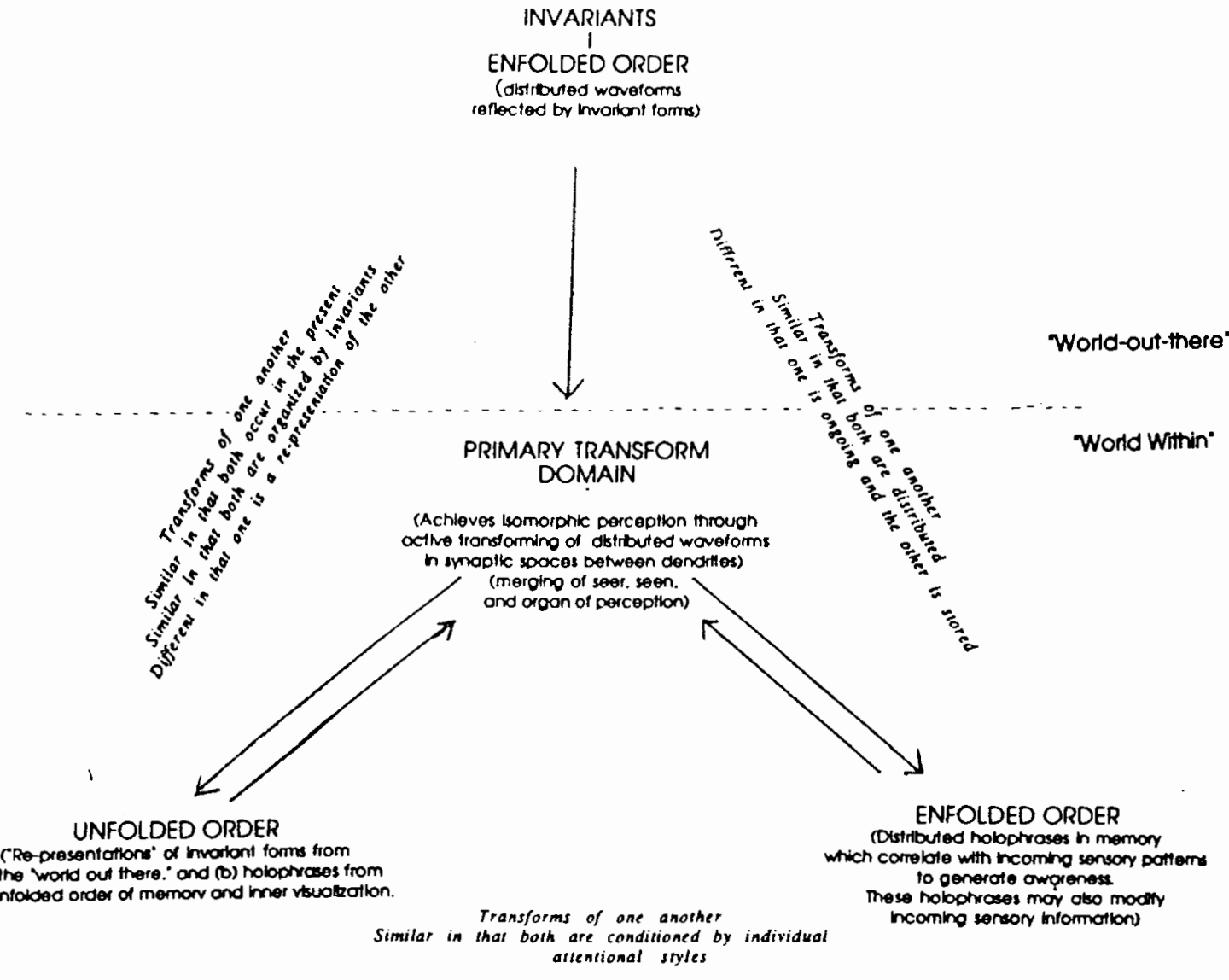

Eigure 4. Constructional realism: A schematic depicting possible perceptual interactions. At the center of the process is the transform capacity of the nervous system which enables algebraic isomorphism through active transforms of vibratory information in the junctional microprocesses (synapses) between dendrites of neurons. 
The Three Domains of Helonomic Constructional Realism

There are three "domains" in holonomic perception which can be inferred from the three aspects named above: the primary tranformational domain, the unfolded domain and the enfolded domain.

1. The primary domain is the inner, ongoing interactive transforms which occur in the junctional spaces between the dendrites as they resonate to information registered by the senses. It is analogous to the engoing process of intersecting waveforms on the holographic plate. Whereas the hologram is one frozen record, the human nervous system accommodates ongoing process. Here the coding of vibratory input takes places. From this emerge two more domains. Pribram calls these the "face-up" and "face-down" aspects of the holonomic model.

2. The unfolded domain is the decoded, re-presented reality which enables one to experience objects, people and events in three dimensional space and time. It also includes the re-representation of distributed holophrases in memory. This re-presented domain is analogous to the decoded image in holography. Through this holonomic representation, one actively experiences both the ongoing array of interacting wave forms that assault the senses and past memory, and combinations of the same. Memory also influences the incorporation of new holophrases and qualifies the process.

3. The enfolded domain has two aspects. The first is enfolded holonomic memory. This is the existing store of collapsed, distributed holophrases--analagous to wave forms on the holographic plate after they 
are recorded. There is no English noun for this domain. Unconscious, subconscious, or memory are approximations that do not, in their everyday meaning, fully convey the correlative and supporting role it plays in human perception and awareness.

The second enfolded aspect is composed of interactive waveforms which exist in nature in distributed form before the senses come in contact and encode and decode them. It is the lensless, distributed order that exists in a buzzing, booming array as interactive waveforms. Pribram describes it as "erganized bouncing of incident radiation off the object so that the organization of the radiation becomes distributed" (Pribram, 1989a, Lecture 3, p. 9). As an alternate example, one might think television signals or radio waves that exist as potential information, but require a reverse transform process to become meaningful information.

To a degree, this distributed wave-form domain corresponds with human memory in that both can be decoded by into the active transform domain into the unfolded order. But memory is different in its specificity, and it exists in latent form throughout the nervous system, beginning with the senses themselves, as distributed information-holophrases--that has been preprocessed via human attention. It carries the unique signature of the attentional "predelictions" and choices of each individual. These holophrases can also affect the processing of new sensory information (Pribram, 1989a, Lecture 4, P. 47; Capra, 1982, p. 295). 
As they are discussed here, the three domains of holonomic perception interact and influence each other. They are of a whole, unlike dualistic notions of reality. Arbitrary distinctions are for the purpose of studying them as an interactive whole so that the basic nature of perception--empathic perception included--can be inferred. This established, this paper will explore the possible principles that govern ongoing processes and interaction in each domain and relate them to empathy.

Holonomic Rerception and Empathy in the Primary Domain

Active, ongoing process characterizes this domain. The real "groundwork" of perception, the activity upon which all else is known, intuited, "re-membered," or inferred takes place in the form of awareness of patterned firing in synaptic space. Without it, the unfolded and enfolded (memory) aspects of perception cannot exist. The raw information that is transformed in the primary domain exists as nature's enfolded active array (described above) of interacting, electromagnetic, vibratory activity that is reflected from "invariants" (a person, an object, word patterns, and so forth). This is registered on the sensory apparatus and modified by top-down, existing "mean" patterns at horizontal connectivities within the nervous system. The primary reality of vibratory interacts is selectively approached by the human nervous system; only certain band widths of the electromagnetic spectrum are processed by each sensory organ leaving other ranges out. It is this sampling that provides raw material for perception. 
Six important principles would seem to influence perception in this domain: holism; algebraic isomorphism which enables a direct relationship between seer and seen through complementation; the enfolding/unfolding of experience via transforms of energy-momentum and space-time; the creation of constancies which result in the effect of perceived form from what was originally process; awareness via dishabituation; and intuitive response via concentration.

Holism. Defined in the first part of this chapter, holism is taken up again to include two additional considerations relevant to the study of empathic process. The first consideration is that the principle of holism is scientific and can be approached mathematically:

I would like to see the label "holistic" become respectable. For not only is the whole greater than and different from the sum of its parts, as the Gestalt psychologists were wont to point out, but the whole can under certain conditions also become enfolded in all its "parts." Thus each part represents the whole, as in a hologram. Convolutional and matrix mathematics, the distributed and dissipative structures we are coming to know, allow holistic descriptions to be as rigorously scientific and precise as any that have been used in physics, chemistry and biology. (Pribram, 1985b, p. 6)

Secondly, the concept of holism suggests that one might understand general aspects of a person's psyche through apprehension of a specific aspect. This underscores the importance of attending to details and nuances in another person rather than assuming similarity (Hastorf, Schneider \& Polefka, 1979) with the other person or indulging in categorical labeling and generalized impressions. If the essences of another person are represented in each part, then nothing about a person--tones of voice, carriage, reaction time, idiosyncratic expressions, attentional choices, smell, and so on--is nonessential 
information. Reik's (1948) "free-floating attention" gains significance in light of this principle, as does his attention to detail:

Free-floating attention provides, so to speak, a storeroom of impressions, from which later knowledge will suddenly emerge. It also creates the prerequisite conditions for those surprising results that appear in analysis as the product of a prolonged unconscious condensation and dissociation of impressions. (p. 172)

Attention to sensory detail is a paradoxical but lawful result of the principle of holism applied to empathic perception.

Isomorphism. The term "iso" is Greek for the word "same," and "morph" refers to "form" (Stein, 1975). The term isomorphism means to have the same appearance. Applied to perception, it means that what is "in here"--the brain--is a faithful representation of what is "out there"--the primary reality. The term isomorphism applied to perception was first used by Gestalt psychologists, especially walter Kohler, who thought the brain had a pattern in it that corresponded to one's perception of what one was actually perceiving, and that what one perceived was isomorphic with internal brain impressions.

Wolfgang Kohler's theory of isomorphism [was] the idea that there was a one to one correspondence between the form (morphology) of the world around $u$ s and the form in the brain representing that world.

Kohler's isomorphism was literal: if one were observing a square, Kohler would expect to find electrical activity in the form of a square in the brain. (Pribram, cited in Goleman, 1979, p. 83)

Brain research has always presumed some kind of geometric isomorphism with outer reality and inner neural circuitry, looking for engrams--neurological representations of outer reality--by probing inside the brain for areas that correspond to things, places, and processes outside the brain. This assumption is important because it 
underlies our faith that the world "out there" and the world "in here" (the brain) are the similar. If so, then directness of perception is assumed to be possible.

Holonomic brain theory also presumes isomorphism, but with a fundamental difference. It introduces an intermediate step, the Fourier Transform, which creates isomorphism that is algebraic instead of geometric. In mathematics, isomorphism is said to exist whenever transformations are readily reversible. Holonomic transforms result in isomorphism with our world. However, instead of isomorphism based on matter directly reflecting matter, algebraic isomorphism is process resonating to process that in turn creates the conditions for the objectification and resultant nominalization of matter. It results in an isomorphism which resonates to and is representative of the original through transforms. At the same time the brain enables us to incorporate these representations into memory "not as pictorial but as self-maintaining structures that act somewhat like setpoints of thermostats undergong [through correlations] continuous adaptive change" (1989a, Lecture 1, page 29).

In other words holonomic theory maintains that our senses encode invariances in our environment in rich and representative ways so we can interact with them dependably; and, importantly, that we embody a shared and universal process in the sense that the Fourier Transform has universal application to the wave-form domain.

In contrast, a dualistic notion reality is based upon geometric isomorphism which assumes just two elements--the seen and the seer. A 
"mind-separate-from-matter" reality is the logical consequence of this assumption. Algebraic isomorphism alters the assumption of separateness. This is accomplished via a mediating element, the organ of perception, which is comprised of ongoing holonomic computations in synaptic space. In the holonomic brain metaphor, three elements interact: the seen, the organ of perception, and the seer. These correspond to the three domains of constructionist reality. In a 1989 interview, Pribram made this distinction about isomorphism:

There are really three things involved--that which is being perceived, the perception as we experience it, and what the brain is doing. Kohler, with whom I worked, never really got straight to my knowledge that there are three things, not just two things... . So we have to try for isomorphism between that which we're perceiving, our perception of it as we experience it, and the brain. So it's three things. Of this, the isomorphism can go beyond what Kohler thought. But then, we're dealing with transforms. So now Roger Shepherd has coined the term "secondary isomorphism," which is a mathematical isomorphism in which you get invertability, which the Fourier Transform does have. Any linear transform is invertible. You can get. back what you had originally by doing the same thing over again, doing the inverse transform. So I call that algebraic isomorphism. (Pribram, 1989, p. 6)

With algebraic isomorphism, one neurologically resonates to information. Dualistic inside/outside distinctions become useful but prosaic terms. What is happening out there is continuous with what is happening in here--unless one creates a selective attentional lens to magnify, modify, or buffer it out.

Because of the concept of isomorphism, a major shift in perception can now occur. One can begin to experience the corporeal self inside out, as a mass of sensitive and intricate, neurological organization immersed in a sea of ongoing, undivided, vibratory events. 
Space now unites rather than divides. What seemed to be "outside" only, is found resonating within. That which is within is unfolded outside. The ordinary world of appearances takes on a secondary nature because one recognizes the primary nature of vibratory interacts. The process is rich and interactive with correlative capacities made possible by innumerable encoded, distributed holophrases that constitute memory.

This perception of self, valid in the holonomic paradigm, can reawaken empathic connectedness with others. Attention gains an enhanced role. Rather than reacting primarily to outer events, humans can become proactive, choosing how to use attention, where to place it, what to ignore. The holonomic brain model can expand these attentional choices by directing one's attention inward to more conscious monitoring of holonomic perceptual equipment.

Attention plays an important role in algebraic isomorphism. Genuine isomorphism--faithful representation--is an ideal that may be difficult to achieve because the three elements involved--seer, seen and organ of perception--may be subject to distortions of memory and attention that obscure perception, including empathic processes. Chapter IV deals with this subject extensively.

Enfolding/unfolding of experience via transformations of energymomentum and space-time. The ongoing enfolding-unfolding aspects of transformations enable humans, through interaction and experience, to neurologically ingest experience, to become the repositories of vast amounts of stored "in-formation" of energy patterns with phenomenal correlative capabilities. The process is scientifically esoteric and 
counterintuitive in its function and implications, but Pribram (1987) has explained it in a paper entitled "Bergson and the Brain: a Biological Analysis of Certain Intuitions." His verbatim explanation is included in Appendix G.

This principle is fundamental in significance to the holonomic brain model because it creates the unfolded experiences and enfolded memory. Its relationship to the empathic process may not seem apparent at first, but the implications are that the enfolding-unfolding capacities of the nervous system enable one to assimilate the ongoing affective states of other person, "out there" and encode the experience directly in one's own nervous system. The enfolded information can then await one in distributed form where inner correlations with other holophrases of experience are possible. In stored, enfolded, memory, it is retrievable for conscious understanding, or for correlations with the next interaction. In distributed form, these correlations are nonlinear, enabling creative insights and unexpected realizations about the other to be materialized back into thought.

As a process, it may provide an explanation for Theodore Reik's (1948) description of empathic incorporation and reverberation, the aspect of empathic process that dives down beyond conscious attention, the part that "interests us most of all" (p. 131). Reik wrote extensively about the ability to listen to another human with great acuity, trusting unconscious processes to reconfigure those impressions into new insights. If Pribram (1987) is correct, then when perception is algebraically isomorphic--when seer, seen and active transforming are 
merged as one event--empathic perception is accurate and direct, and it avails one of the vast stores of enfolded information in memory. Both Reik (1948, pp. 131-213) and Pribram (Goleman, 1979, p. 84) suggest focus and attention as keys to retrieval of the enfolded to the unfolded in vital form. Isomorphism may provide for directness of perception of ongoing sensory events. Pribram (1984c) refers to this experience as "primacy of experience" (n.p.).

creation of constancies. Interference patterns create "constancies" which, in turn, enable perception of form from what is actually process. Constancies are stable interference patterns that repeat and sustain themselves during ongoing experience. It is these constancies which also become distributed holonomically throughout memory. In a 1984 paper entitled, "Science and the Mind Brain Issue," Pribram writes,

Since parts of objects as well as whole objects serve as sources of reflection and thus as references for other parts, constancies are generated when images are (re) constructed. Constancies are therefore the result of the fact that the transformed "view" of any part of the objects acts as a reference for every other part. (1984c, n.P.)

The principle of ongoing process becoming form through constancies serves both the face-up and face-down aspects of the model in specific ways that deserve consideration here.

Holographic patterns in the primary domain are both process and form. These patterns of densities take on qualities--color, form, movement, intensity--that in turn create constancies. What was first process--a buzzing, booming, vibratory interact--congeals 
holographically to "in-form" one's nervous system while interacting with previously experienced constancies. The term congeal should not be misleading and is used here only for the lack of a better one. Congeal implies hardening to form. This term is applicable to memory in the enfolded domain, but not to perception in the primary domain, because patterns here are in a fluid, active state. What appears to congeal is initially a relatively constant patterned state that is sustained by ongoing activity, similar to the effect of standing waves in the ocean when observed from a high altitude.

In the face up, unfolded domain, the creation of constancies may also underlie our human proclivity for nominalizing what was originally process (Hall, 1959, 1976). Pribram underscores this possibility, calling these "standing waves" holophrases and explaining that reification--the perception of objects as separate things--evolves from order invariances across transforms:

This property of mind to maintain constancies across transformations is, I believe, responsible for reification, the natural tendency to objectify what are initially experienced as processes. . . Propositions grow from holophrases which symbolize existing situations, both external and internal. Also natural is the tendency for the holophrases to become nominalized, i.e. to make objects of the situations symbolized. Thus a symbol which originally referred to a process, tends later to refer to an object. (1984C, n.p.)

This process is reflected in language:

"In the beginning was the verb," i.e., words originally referred to a flow of experience, early communication was "verbal"! The word "word appears closely related to the word "verb." At a recent conference.. . we were appraised of the fact that initially Hebrew words were verbs denoting being, action and process. A similar form is said to exist in preclassical sanskrit. Be that as it may, there is every 
evidence that human thought, including scientific thought, begins by nominalizing. (Pribram, 1979a, p. 2)

Many hierarchies of constancies exist as holophrases in ordered stages throughout the nervous system--echoing the ancient "as above, so below" axiom. First, ongoing interference patterns are registered by the senses. As they interface with existing patterns encoded in memory, newer and more stable constancies are created. Ascending stages of horizontal interconnectivities integrate these patterned constancies into ever larger sets. The cerebral cortex incorporates these into still more stable, more global sets which, in turn, provide for computation with incoming patterns. This is oversimplified, but the point here is that the nervous system accommodates constancies in ever larger and stable patterned states, with feed-forward, feed-back, interacting design (Pribram, 1971).

Ignorance of the principle of constancies and resultant nominalization or reification may result in inefficient use of attention. To the extent that attention is given over to secondary form without awareness of the basis of that form, one abdicates awareness of ongoing process and choices about patterns and constructs. An inward shift of attention returns one to process and increased choices of how to use attention. From this center, one's perception can be more isomorphic. Perceptual constructs can be experienced as constructs, enabling one's choices to be more proactive than reactive. This distinction is not a trivial one. In his book A Theory of personality: The Psychology of Personal Constructs, Kelly (1955) explains:

It [choosing how to represent one's environment through choice of constructs] emphasizes the creative capacity of 
the living thing to represent the environment, not merely to respond to it. Because he can represent his environment, he can place alternative constructions upon it and, indeed, do something about it if it doesn't suit him. To the living creature, the universe is real, but it is not inexorable unless he chooses to construe it that way. (p. 8)

This awareness of choice has significance for the ability to listen empathically. By withorawing the center of attention from one's senses and quietly stationing it inward to monitor ongoing process, attentional choices can become more flexible and volitional. One is able to "melt down," transcend, alter or recreate limiting constructions about reality; or perhaps one can simply see his own and another's constructs as they are. The key here is to understand the process behind the forms.

The enfolded domain of memory, however, is enriched by the constancy principle. It makes patterns and correlations, memory, and mind possible. The next principle describes how awareness, also, may be generated from constancies.

Awareness via dishabituation. When the "fit" of new information does not match past experience, attention is awakened. ongoing holonomic processes create dissonance when the nervous system receives patterns at variance with the range mean of patterns stored. When this occurs dishabituation results and awareness is activated, resulting also in an orienting reaction.

Dishabituation to novelty engages the junctional and dendritic mechanisms of the brain where the slow potential microstructure, the holographic representation of the input, is produced. Only with repetition do patterns of these slow wave potentials intercorrelate sufficiently to generate the nerve impulses necessary to action. Each slow potential pattern is assumed to leave its residue at these synaptic junctions and dendritic locations and so participate in generating the correlations. In short, to the extent that 
our experiences fail to correlate, to the extent that our actions are uncontrolled by habit, to that extent they are voluntary and we are conscious. (Pribram, 1976, p. 306)

A little imagination here results in a picture of the nervous system as a dynamic generator of awareness. Correlations therefore awaken one. The process of dishabituation can result from external or internal dissonance, from difference in patterns entering the system through sensory experience or from alterations of internal awareness.

Intuitive response via concentration. One might be able to attentionally access the correlative potential of the enfolded, "unconscious" memory by either holding a steady-state visualization (i.e., looking for a face in memory) or by holding a steady-state question (i.e., awaiting a coherent understanding regarding the unknown explanation for another's irritating behavior) until the "invariant" holophrase appears in its own time in the inner attentional space created for it. These kinds of pointed concentration might access the enfolded store of distributed holophrases distributed throughout the nervous system. If this is possible, then nonlinear correlations could speed response to one's visualization or question, and introduce nonlogical, creative answers, associations and ideas into consciousness. Samuel Ghosh, (1980) author of The original Yoga, a text explaining Patanjali's Sutras, explains that principle above is basic to Eastern Yogic tradition. In a letter he responds to a written query about the relationship between yoga, union, and empathy:

Empathy and even supernormal powers like mind-reading, telepathy, clairvoyance, clairaudience, etc. can be gained this way. For as we now understand, consciousness in human beings is dispersed all over: it is held incommunicado in the billions of cells that form the somatic entity. 
Patanjali suggested that this diffused consciousness should be concentrated and brought to a point. Such intense concentration or ultra density of thought results in a flash-point, and there is then a sudden light of direct knowledge--that is, without the aid of reason, discrimination et al. We now call it intuition. Inventions and discoveries are often the product of such flashes of intuition. Incidentally, the Sanskrit word $\mathrm{Rg}$ (rc) literally means "a flash", "a spark," when there is a sudden awareness in the mind of the truth or reality. (S. Ghosh, Personal communication, oct. 23, 1986)

His explanation articulates a process similar to that described by Reik

(1948) in Listening with the Third Ear.

A symptom, the fragment of a dream, a long-forgotten fact suddenly appears in a new light; widely scattered data arrange themselves in a new relation. Retrospective reflection shows that unconsciously we had understood the the meaning of the separate facts very well, and that this unconscious comprehension was, so to speak, a necessary preparation for the present surprise. Things that were not in the center of our attention, things that were at the fringe, a passing impression, a fleeting presentiment, now take on importance. (p. 172)

It seems apparent that there is no way to access distributed

information linearly. Reik observes, "attention of that special voluntary kind prevents surprise. People who practice it always find confirmation of what they already know" (p. 170). Rather, it is necessary to approach the unfolded store indirectly through concentration in the present, posing questions or holding inner visualizations, trusting that the enfolded domain will be active and respond, referring the insight to consciousness.

In other words, the psychoanalyst who hopes to recognize the secret meaning of this almost imperceptible, imponderable language has to sharpen his sensitiveness to it, to increase his readiness to receive it. When he wants to decode it, he can do so only by listening sharply inside himself, by becoming aware of the subtle impressions it makes upon him and the fleeting thoughts and emotions it arouses in him. (P. 147) 
If Ghosh (1986) and Reik (1948) are correct, then concentrated attention in the primary domain can reach the enfolded, distributed domain and order specific information. This information is later unfolded to conscious awareness, complete with inner visualizations, new correspondences and redistributed significances. These instructions for creative and intuitive thought are not new, but the holonomic model provides structure that helps explain them as basic principles of perception.

This principle of holonomic perception pertains to the reverberation and detachment stage of Reik's description of empathic process. It also takes the empathic process a step farther. One trusts that the information about the other person is already incorporated into the nervous system during the listening and attunement of the identification and incorporation stage. This information, once assimilated, is beyond the purview of ordinary waking attention, but is reverberating throughout the distributed holonomic store of patterns in personal memory. Because correspondences here are nonlinear, new insights and configurations of "in-formed" experience can refer themselves back to consciousness, ordered up, perhaps, by the steadystate, focused questions posed to the "unconscious" mind. This information would not be in words, but rather in insights, pictures, feelings and intuitive hunches.

Reik's (1948) descriptions of empathic listening stress the importance of turning one's ear of attention inward to catch this silent but felt insight as it occurs. New understanding is the payoff of an 
earlier investment of attention. However, the process of reverberation takes its own course. Insights are gestated in the distributed domain and birthed in the primary domain through attention. Reason is not applicable in the distributed domain, but when insights refer themselves to consciousness, inference from the new information is possible. In this way knowledge is enhanced.

This section has explored potential principles of perception that emerge from active transforms of interfering wave patterns in the primary holonomic domain. The next two sections explain possible principles and interactions in the unfolded and enfolded domains. In holonomic theory, these domains are secondary because they result from transforms in the primary domain.

Holonomic Perception and Empathy in the Unfolded Domain

Some principles of the unfolded, face-up domain have already been discussed in the first portion of this chapter: three-dimensional image construction and the experience of time and motion; the projection of images away from the source; and experienced based upon shared process. This section will explain additional principles of perception in the manifested, unfolded domain that apply to empathy. These are: anisomorphic perception as everyday consciousness; objectifying via movement; use of mimicry and synchronization to intuit orderinvariances;" and kinesthetic awareness and a corporeal sense of "I." Anisomerphic perception as everyday consciousness. The previous section explained the central nature of isomorphic perception to direct knowing. However, perception can also be anisomorphic. "Aniso" is a 
Greek term meaning "unequal" or "uneven." The term refers to perception of form that is not consonant with actual ongoing sensory information. Because perception involves internal as well as incoming sensory holophrases, it can be affected by pre-existing memory that is decoded into active three-dimensional space, overriding or modifying actual sensory perception.

This is accomplished in part by convergences and divergences of pathways but even more powerfully by networks of lateral interconnectivities, most of which operate by way of slowgraded dendritic potentials rather than by nerve impulses propagated in long axons. (Pribram, 1980c, p. 8)

Pribram refers to the mechanisms that create anisomorphic perception as "re-presentational" mechanisms (1980c, p. 4):

Ordinarily an organism's re-presentational processes are called images, and there is no good reason not to use this term. But it must be clearly kept in mind that the perceptual image, just as the image, is more akin to a computation than to a photograph.

The perceptual image so defined is therefore a representation, a mechanism based on the precise anatomical punctate receptor--cortical connectivity that composes an array. This array is operated upon by lateral

interconnections that provide the ambiences which process the invariances in the organism's input. Thus the cortical re-presentation of the percepts go therefore beyond the anatomical re-presentations of the receptor surfaces just as the cortical re-presentation of actions goes beyond the mere anatomical re-presentations of muscles. (Pribram, $1980 \mathrm{C}$, p. 8)

For example, consider the experience of the phantom limb. The amputee feels his leg or arm as if it were still there. Its objectified, felt presence occurs because its kinesthetic sensory pattern is encoded holographically in his nervous system. This holophrase can be reprojected out away from the body--whether the actual limb is there or not. "There is thus incontrovertible evidence that re-presentations of 
prior experience with the environment are involved in the processing of sensory input" (Pribram, 1980c, p. 10).

We usually assume that what we feel is really out there, but holonomic brain function works both ways. We also project outside ourselves what is holonomically imprinted within us, and it can be mistaken for direct perception. The phantom limb experience is used as an example in the last paragraph because it is well known and graphic. In the holonomic mind, it exists as a kinesthetic holophrase encoded into the dendritic arena through past experience. The senses, spine and brain contain innumerable sensory impressions, memories and constructs in the form of encoded holophrases. These internally driven holophrases, superimposed and unfolded over fresh experience, can result in anisomorphic sensations--perceptions that are not direct and isomorphic.

Thus the information is not just a property of the array but is also a function of the transforming and correlating powers of the organism. This is an extremely important point to grasp: in one view (Gibson's) the organism is a passive gatherer in an object world; in the other (the brain scientist's) the organism is actively involved in the construction of this object world. (Pribram, 1983a, p. 33)

Anisomorphic discrepancies are so basic to everyday habits of perceiving that their significance is easy to miss. Discrimination is necessary to know whether perception is direct or anisomorphic. This discrimination can be aided by the understanding that unfolded holonomic transforms from memory project re-presentated holophrases outward, apart from ourselves, to be experienced as objective and immediate experience. 
Direct, isomorphic experience of unfolded reality may be possible when the holonomic mechanism is sensory driven (Pribram, 1989. p. 9); but when it is internally driven--i.e., when we respond primarily to decoded engrams from memory, or dreaming, or impose inner constructs, or respond to what is internally visualized, rather than what is there-then perception is anisomorphic. Here one should not confuse inner holophrases with inner witnessing awareness, the two concepts are distinctly different. Holophrases are encoded patterns; inner witnessing awareness is unqualified attention that can also attend to new sensory information.

Given the above explanation, it is possible that even if the internal holophrase is "correct"--that is, one perceives one's limb and it is there, in fact--direct knowing does not necessarily occur. Even then, when the perception is verified externally, one's experience of it may not be a direct one. A reinvestment of attention to ongoing sensory experience--temperature, pressure, and so on--could result in isomorphic re-perception. This investment of renewed awareness to sensory data may be necessary for the amputee to know the leg does not exist, even though the internal construct of it persists. Therefore, given the holonomic hypotheses, one can see without "seeing" or hear without "hearing"-resonating to the "same form"--if one is attuned to primarily inner holophrases and does not actively attend new incoming sensory data.

The above discrimination is important to the training of accurate empathic perception because anisomorphic perception distorts the process. This principle gives the underlying reason why judgmentalness, 
personal agendas, expectations, past experience, and limiting conceptual attitudes inhibit empathic awareness. These are all examples of inner holophrases that create anisomorphic perception when superimposed over actual interaction with another person. The problem here is that one will come away from the interaction knowing only what he knew before. objectifying via movement. An interesting fact about the brain is that all the senses are surrounded by motor connectivities, and the human nervous system has more progressive separation of motor from sensory cortex than do animals (Pribram, 1983b; Pribram \& Carlton, 1986). Assuming that this configuration serves perception, one asks "why?" Pribram answers this question with the following suggestion:

Have someone touch you with a pencil or another object. You feel the touching, rubbing, pressure, etc. These are elementary qualities of tactile sensibility. Now grasp and rotate the same object in your palm by active manipulation. suddenly the object, e.g., a pencil, has materialized! $(1983 \mathrm{~b}, \mathrm{p} .1384)$

The motor-surround connectivities perform a basic function for direct knowing. Because of them humans can "feel" around the target object, examining it from several perspectives.

objectivity [creating objects from processes] apparently results, not only from the lens-like structures of the senses, but also from the constancies, the invariances, culled from the variegated interactions between the senses and [that which is] sensed which result from movement. Correlations, facilitated by the holographic microstructure of the sensory systems, play a critical role in establishing invariances. (Pribram, 1984b, p. 29)

This function is fundamental to behavior. It is used on levels of perception from sensory to conceptual. Pribram writes: "objects are perceived when the organism actively moves about the environment-whether with his eyes, hands or whole body" (1983b, p. 1386). A new 
idea is "examined from several perspectives." What may not be apparent is a possible deeper function of this behavior. By examining an object from several perspectives, some aspect of it stays constant. The information which stays constant, the part that does not change, is targeted as an unknown "it" and objectified. In a real sense we know what something is by knowing what something is not. Human perceptual behavior seems to vector in on that which is invariant, by "in-feeling" to the source (which echoes Lipps use of the term einfuhlung--"in feeling" (Appendix B).

Use of mimicry, and synchrenization te intuit order-invariances. The explanation above may also underlie the purpose of motor mimicry, an innate empathic behavior observed even in newborn babies (Appendix B):

The process occurs when, upon observing the stimulus person's facial signs of distress, the child automatically imitates these emotional expressions by engaging in slight movements in facial expression and posture. These imitative movements then cause inner kinesthetic cues that aid the child in experiencing and understanding this same feeling. The existence of this mode of empathic transmission, as well as its possible innate origins, has been given support recently in an exciting group of infant studies showing successful motor mimicry of facial expressions during the first months of life. (Barrera \& Maurer, 1981; Field, Woodson, Greenberg, \& Cohen, 1982; Nelson, Morse \& Leavitt, 1979; Young-Browne, Rosenfield, \& Horowitz, 1977). (Goldstein \& Michaels, 1985, p. 15)

Mimicry enables one to synchronize behavior with another person, but the holonomic model suggests a deeper purpose for this behavior.

Through mimicry one may be able to attune to and understand kinesthetically what is variant and invariant. By synchronizing movement and timing with another human's movements and reactions, perceptual valences are created; one becomes aware of something unknown. 
The perceiver can alse intuit nameless order-invariances, or holonomic patterns that do not change in the midst of changing patterns. The constancy may be a mood, attitude, or even an absence of a previously internalized constancy (i.e., mother is not relaxed). Consequently, in a motor sense, one could know holonomically what something is by knowing what it is not. This theme of knowledge via order-invariances across transforms is consistent throughout the range of holonomic perception, from incoming sensory details to concepts.

It may also be human nature to be uncomfortable when something objectified or unconsciously identified as an order invariance across transforms is no longer invariant (i.e., the baby cries!), sending one in search of another constant. This search may help drive the internal dance of synchronicity and mimicry that comprises much of human interaction. It is important to Reik's (1948) identification stage in empathic process.

Once an unknown invariant is intuited through movement, one can concentrate "with question" on it. As explained in the principles of the primary domain, if one attends to a question with sufficient concentration, correlations in the distributed domain of unconscious memory may serve up an intuitive response. The previous unknown--in this case the nonverbal invariant discerned through mimicry--is then introduced into the unfolded order. Thus one "real-izes" something about the other person and it is encoded directly into one's neural apparatus. This phenomena could describe the core of the empathic process. 
However, the inference that humans can understand another's inner, affective states through mimicry and synchronization is based on the assumption that emotions are both universally experienced and objective in their meaning. If not, then mimicry and synchronization cannot enable one to reliably experience or intuit another's feelings. If so, then the principles described above may have merit.

Before continuing this line of reasoning, a distinction should be made between the body's affective response and the meaning of that response. The first would appear to be more accessible empathically through mimicry than the second. The second requires awareness of both the cause of the affective response and the personal and cultural constructs of the other individual. These are more difficult to intuit directly. However, this disclaimer does not lessen the underlying effectiveness of the principle described here or its usefulness to empathic indentification and incorporation.

Nathanson (cited in Brain/Mind Bulletin, 1991b), a Pennsylvania psychiatrist, recently provided a description for the anatomy of the human affective system which may validate the assumption of objective emotional states. He profiles affects--displays of emotion--that are triggered by specific patterns of "stimulus density," the rate and intensity at which data are presented to or processed by the brain. Each of these innate affects appears on the "display board" of the face Facial muscle patterning, unique to each affect, occurs even when invisible to the eye. Even finger movements have a measurably different quality with each affect. This would imply that innate affect is much more than a vehicle for [ordinary] interpersonal communication. (Nathanson, cited in Brain/Mind Bulletin, 1991b, p.7) 
Nathanson describes a biological profile of nine affects originally named by Silvan Tompkins (1991a, cited in Brain/Mind Bulletin) in his book Affect Imagery Consciousness. These nine affects are: interestexcitement, contentment-joy, surprise-startle, distress-anguish, fearterror, anger-rage, dissmell, disgust, and shame. ${ }^{4}$ He adds that in combination they provide an emotional palette from which a wide range of emotional meaning is conveyed.

The palette of affect produces interesting combinations. Contempt, for example, might be a fusion of anger and dissmell, sneer a combination of dissmell and disgust. (p.6)

William James (1991b, cited in Brain/Mind Bulletin) first proposed that emotions result from "bodily reverberations" rather than the mind. This observation, developed further by Tomkins and Nathanson (cited in Brain/Mind Bulletin, 1991a, 1991b, respectively), is relevant to the theory of holonomic empathy because it cuts out an intervening variablethought--and by doing so, makes emotional affect more directly accessible to perception via mimicry and synchronicity.

My theory. . . is that the bodily changes follow directly the perception of the exciting fact, and that our feeling of the same changes as they occur is the emotion. [By normal interpretation] we lose our fortune and weep; we meet a bear, and are frightened and run; we are insulted by a rival, are angry and strike. This order of sequence is incorrect. The one mental state is not immediately induced by the other. The bodily manifestations must first be interposed between. The more rational statement is that we feel sorry because we cry, angry because we strike, afraid because we tremble... .

Without the bodily states following on the perception, the latter would be purely cognitive in form, pale, colorless, destitute of emotional warmth. (James, cited in Brain/Mind Bulletin, 1991b, p.6)

Thus the theories of emotion proposed by Tomkins, Nathanson, and James provide a foundation supporting the assumption of shared human affect. 
If their observations are correct, then it is possible that holonomic perception enables us to directly experience the ongoing internal states of others through their outer signs (facial and body affect), and also, to discern unknown internal states through patterned, holonomic constancies that are intuited through the mimicry and synchronicity. The personal constructs which determine values and meaning may be more difficult to intuit, although they may influence visible affect. Inference on the part of the observer may be required to discern them. Nathanson also describes an "empathic wall" which can interrupt or consciously buffer attunement to another person's affective states. This takes the form of reinforced personal constructs to shut out the new information, or the avoidance of the new experience (cited in Brain/Mind Bulletin, 1991b, p. 7). Such a "wall" may actually be desirable if empathic attunement might result in unpleasant or unhealthy effects upon the nervous system of the perceiver. However, it is inhibiting if the intent of the perceiver is to be open to and to understand the other person.

Kinesthetic awareness and a corporeal sense of "I". An important face-up aspect of holonomic capacity for creating the effect of felt form is the possibility that our ordinary sense of self, the rather ethereal and hovering aspects of personal presence independent of actual body boundaries, may be holonomic. Pribram suggests that the same capacity for giving three-dimensional locus to feeling and projecting it out from ourselves, may be responsible for the personal felt experience of the "ghost in the machine," the phantom sense of "I". Thus the 
holonomic capacity for reifying process described earlier might provide the comfort and assurance of a perceived sense of self.

The holographic hypothesis also provides the most plausible explanation for consciousness of self, that undeniable experience of standing out as personal projects who "mind" what happens. If vibrators modeled on the human ear are placed on each forearm and their frequencies tuned, a point of sensation will at first jump periodically to and fro between them. But after habituation the sensation point moves midway between them. Here, then, is a "feeling self" and embryo "mindscape" created out of synchronized wave forms, with the individual able to project a self outward like the sound waves aimed by two stereo speakers. (Hampden-Turner, 1981, p 97)

This sensory self would project a comforting sense of corporeality beyond the body. In theory it is different from pure, undifferentiated inner awareness; rather, it would take the form of residual awareness that is localized holonomically via attention.

Kinesthetic awareness would also seem to be plastic in the sense that it is holonomically malleable. It can explain the capacity for altering perceived body boundaries and may be used to extend one's limbs beyond their natural limits, as is evidenced when one uses tools. The screwdriver, for example, is mastered only after one learns to project and fine tune kinesthetic awareness to its end. Once the skill is learned, the process of changing one's body boundaries to include the end of the tool is usually unconscious.

The use of tools is only one application of the use of holonomic kinesthetic awareness. It can be projected into both static and moving objects. The body language observed as sports fans watch and respond to athletic events is an example of expanded kinesthetic boundaries. It is also possible that by altering boundaries kinesthetically, one can 
accomplish the "in-feeling" to experience the other person during the identification and incorporation steps of empathy.

In Empathic Perception: The operation of Self-Awareness in Human

Rerception, Bennett (1972) includes a lengthy discussion of kinesthetic awareness, observing that "both projected body awareness and body extension are devices through which we perceive objects empathically" (p. 97). He explains why it may be easier to empathize kinesthetically than to intuit another's values and meaning constructs.

The idea of projected body awareness gives another clue as to why body empathy is so easily achieved. Most of us have a relatively high degree of body awareness. We know, for instance, quite precisely whether our bodies will fit through a given doorway or not. Most people can touch their noses with their eyes shut. We all evidence some rather remarkable coordination in walking, taking showers, and generally moving around in our environment successfully. These things indicate that we are fairly aware of our body size, the position of various parts, and how they work together. It is very likely that we know more about the configuration of our bodies than we know about the configuration of our world views. It is therefore not surprising that we are more attuned to other people's body experience than we are to other alien experience. (pp. 9192)

A distinction between body affect and the meaning of that affect was also mentioned earlier in the discussion of mimicry, synchronizing and order invariances.

Kinesthetic awareness may thus be a source of practical physical and psychological effects. It may also be food for the sense of "I." It may be natural to identify with kinesthetic awareness, because one uses it to control and experience one's environment. However, a distinction should also be made between this kind of self-awareness and the more internal watching awareness. Both would appear to be necessary 
to empathic perception. The identification of one's self with the holonomically focused kinesthetic awareness alone could be limiting because kinesthetic boundary changes can be unconscious as well as conscious. The kinesthetic sense of "I" is active and moving and as such it is incapable of sustained focus. In theory, it can be found to be distinct in concept from quiescent, witnessing awareness in inner attentional space, which can watch it.

\section{Holonomic Perception and Empathy in the Enfolded Domain}

The enfolded domain itself has two aspects: it exists internally as stored memory, and also externally in nature as distributed interference patterns that are not transformed

Holonomically distributed patterns in memory are stored throughout the nervous system. What was originally process in the primary domain has become memory and previous experience. These stored holograms enable one to reverberate to and to re-perceive objects, people and events in unfolded, three dimensional space and time, such as the phantom limb or the Malaysian man described in the preface of this paper. Through memory and attention this order may be most contiguous with individual human consciousness. In addition to memory, the distributed store helps generate awareness, as explained in the principle of awareness via dishabituation.

The enfolded domain--in memory and in nature--does not answer to Newtonian, space-time, causal laws. It obeys its own rules that may mirror those which scientists use to describe quantum reality. Thus the face-down aspect of the holonomic model takes us to the lensless domain 
of encoded interference patterns. Dr. Pribram (1984c) explains the enfolded order in an essay entitled "Science and the Brain Mind Issue:"

There is good evidence that there lies another class of orders behind the ordinary classical level of organization, which we perceive and which is described in terms of Euclidean and Newtonian views, and mapped in Cartesian space-time coordinates. This other class of orders is constituted of fine-grain organizations which describe potentials which had been poorly understood because of the radical changes which occur in the transformational process of realization. When a potential is realized, information becomes unfolded into its ordinary space time appearance; in the other direction, the transformation enfolds and distributes information as this is done by the holographic process. Because work is involved in transforming, descriptions in terms of energy are suitable. Because the structure of information is what is transformed, descriptions in terms of entropy (and negentropy) are suitable. Thus complete understanding involves at least a dual: On one hand there are enfolded orders manifested as energy potential: on the other there are unfolded orders manifested in negentropic space time. (n.p.)

Here also, in the enfolded order of memory, there may be correlative interacts. "Correlations are the hall mark of processing in the enfolded order" (1984c, n.p.). These may be totally counterintuitive to us because "In this domain, space and time become enfolded; only density of occurrences is represented" (Pribram, 1986, p. 518). David Bohm (1979), a physicist from London University who worked with Einstein, expands upon the holonomic paradigm by explaining that reality cannot be understood without the concept of two orders of reality--the manifest and the unmanifested world. He calls these the explicate and the implicate domain. The explicate domain is the manifested world of appearances in space and time. He explains that the explicate world manifested "out there" is a ripple of restless, unfolded energy riding on the more primary implicate order. What is out there in 
the unfolded explicate has a "tendency to be." What seems to be real and tactile is actualiy an ongoing, continuous flowing event between the two domains. An oak tree, for example, appears to be constantly present, but it is actually there and not there, blinking "on" and "off," disappearing and being replaced by a different order of oak tree continuously in a constant interplay between the unfolded and enfolded worlds. The implicate order is a lensless, fine-grained, quantum-order reality where correspondences are nonlinear.

Bohm's contribution is mentioned here because Pribram recognized in Bohm's work that the implicate domain may correspond to the holonomic enfolded order in nature. Therefore, when describing Bohm's model or comparing Bohm's theory to the holonomic model, Pribram articulates information that is useful to the understanding of principles of the enfolded holonomic domain. For example, Pribram explains:

He [Bohm] suggests that all of our conceptualizations in physics (as opposed to experimental manipulations and their formal mathematical treatment) are based on the use of lenses. We have telescopes and microscopes which contain objects which objectify. Objects are particulate, separated from one another and can thus move with respect to one another to create the appearance of space, time and causality, i.e. the explicate domain. Take away lenses and one is immersed in the implicate order. (Pribram, 1979a, p. 28)

In a later article, Pribram (1980d) again refers to Bohm's theory and relates it to the holonomic brain model:

David Bohm has noted that, although the mathematics of relativity and of quantum theory are thoroughly worked out, the conceptual representation of what that mathematics might mean has lagged seriously. He suggested that this lag is owing to our propensity to use lens systems to construct our conceptual reality. He proposed that the hologram might provide a better conceptual model for understanding both the macrouniverse and microuniverse. His proposal strikes a 
responsive chord in the neuroscientist who also has found a level of organization in the nervous system that is more appropriately modeled by the hologram than by the senses (i.e., lenses). (p. 33)

Pribram (1971) recognized in Bohm's theory the possibility that the finer-grained, lensless implicate reality corresponded with the holonomic enfolding capacities of the nervous system.

The reason for looking at quantum mechanics for mathematical treatments of neural holographic processes is that the issues faced at the micro-physical level are in many respects similar to those encountered in current neurophysiology. Thus David Bohm. . . has suggested that a holographic-like order which enfolds space and time underlies the observations of quantum physics. Bohm calls this an implicate order to distinguish it from such explicate, explicit orders as those represented by Euclidian geometry and Newtonian physics.

On the basis of these results and formulations the problem of brain-percept isomorphism takes on added complexity. The brain cortex resembles a spatial filter (Movshon, et al, 1978, 1979), resonator or interferometer (Barrett, 1969), a musical instrument or hologram constructing percepts. Such an instrument is not a geometric isomorph of the precepts it constructs. Rather, the isomorphism is seen to be between the brain as an instrument and the arrangement of physical energies elsewhere in the universe. The isomorphism is between two "physical" entities, "brain" and "world" rather than between either of them and our percepts. (Pribram, 1979a, pp. 2728)

The senses, through their holonomic enfolding of that information, may create a transformational door through which the implicate, unmanifested world can be approached. Here, says pribram, the delicate business of intuitive perception "may be accessible through attention in the implicate" (Goleman, 1979, p. 80). Additionally, the lensless domain of memory may resemble the lensless domain of the implicate reality in that both are distributed orders accessible through the transforming capacities of the dendritic computational space of the nervous system. 
It would be presumptuous to try to fully describe principles of the enfolded domain. Words and linear thinking would be alien here. But the list below may help contrast it from the unfolded domain and relate it to other aspects of constructional reality. These principles include Pribram's list of quantum order principles: creativity and nonlinear correlations; accessing the enfolded domain by attention in the active transform domain; and attention as a lens in the enfolded order.

\section{Density of events, synchronicity, symmetries, complementaries and}

dualities. Pribram (1978b) offers the following considerations as working principles in the enfolded domain:

This domain deals with the density of occurrences only; time and space are collapsed in the frequency domain. Therefore the ordinary boundaries of space and time, locations in space and in time become suspended and must be 'read out' when transformations into the object/image domain are effected. In the absence of space-time coordinates, the usual causality upon which most scientific explanation depends must also be suspended. Complementarities, synchronicities, symmetries, and dualities must be called upon as explanatory principles. (p. 17)

If he is correct, then quantum physics can teach us more than is presently understood about the nature of the "unconscious" mind and the laws that govern its interaction with the waking consciousness. Because empathic reverberation occurs beyond the conscious purview of the mind, these become interesting as possible explanatory principles.

Nonlinear correlations and creativity. "Correlations are the hallmark of processing in the enfolded order" (Pribram, 1984C, n.p.). This is because "correlations are much simpler to accomplish in the waveform domain: they essential entail superposition, addition" (1982a, 
p. 277). Because encoded transforms are distributed and spread throughout the nervous system in memory, a vast repository is in place with enormous potential for nonlinear, non-logical correlations. These make possible new creative combinations and effects.

Holonomic storage per se allows myriad novel combinations to be extracted from the brain. . . changes in interpretation of history, departures from accepted theory in science, inventions of new machines and of other cognitive commodities become possible. (Pribram, 1983a, p. 37)

Cause and effect do not apply here, only density of events. Correlations make new densities and combinations possible.

Enfolded domain accessed by attention in the primary transform domain. This principle is described previously in primary domain principles as intuition via concentration in the primary domain. Mention of it is included here because it also involves the enfolded domain of memory. Concentrated attention through steady-state visualizing or questioning may be the interactive bridge between the two orders.

Attention as a lens in the enfolded, distributed order. Expanding on the principle above, one can reconsider Pietsch's (1981) explanation of unusual effects achieved by combining lenses and holography. Again, if one thinks of attention as a lens, the concept may be worth some exploration. Quality of attention may be a determining factor in how one retrieves information from the distributed domain. If the lens of attention is clear, sustained and focused, then the re-perceived memory may be true to the original perceptions. However, if the attention has distortions or cannot be sustained continuously, then unusual retrieval 
effects that are anisomorphic to the original input may occur. This may affect the reverberation aspects of empathic process. Unconscious use of attention is discontinuous, moving about; conscious attention can be more continuous and stable. To the extent that one is awake and aware in inner space, the results of primary experience in the store of distributed memory may be accessible, although not directly. However, to the extent that there are aberrations in attentional focus, anisomorphic impressions may result in the form of fantasy and distortions of previous perception that are then overlaid on ongoing experience. Here again, is the theme of attentional control that has played throughout this chapter.

Exploring the concept farther, there may also be differences in the use of sustained, focused attention, and the use of sustained pure, undifferentiated attention. The first may effect specific retrieval, and the second may tap into the more holistic, global aspects of the distributed domain. This may be the difference between remembering a face or name, and feeling the interconnectedness of events.

If Pribram and Bohm are correct, Bohm's explicate/implicate order and the holonomic model are related. If so, then the transform principle we embody could also put us in contact with all of nature, giving us the theoretical potential of being direct, conscious intermediaries, perceivers and interpreters of vibrational events at a level more basic than concepts or words. The process may also give us access to the deepest aspects of another's experience, if we learn how to listen and respond in ways relevant to the new paradigm. 
Suffice it here to say that I believe the discovery that certain operations of the brain can be understood best in terms of processing in the wave-form domain is as important to the mind/brain problem as the discovery in quantum and nuclear physics that ultimately the appearances of matter may be immaterial. (Pribram, 1979b, p. 117)

\section{ATTENTION AND EMPATHIC PERCEPTION}

A basic shift in attention would appear to be necessary in order to utilize the above suggested principles for empathic perception. If the core process takes place in the primary transform domain, it would seem sensible to maintain attention within. From here, one could key into the ongoing vibratory interacts by allowing residual kinesthetic awareness to move outward and interact with the other person. Poised inwardly, attention would be in a mediating, pivotal place. Theodore Reik (1948) in Listening with the Third Ear underscores the need for the withdrawal of attention from the space-time domain to inner attentional space, saying "the withdrawal of attention does not give rise to inattention, but to a shifting of attention, to a readiness to receive a variety of stimuli emerging from the unconscious or the unknown" (p. 168-169). From this vantage point, the witnessing awareness--Reik calls it poised attention--may have access to two orders, the unfolded domain and the enfolded domain.

Poised attention generally involves the renunciation of the immediate recognition of links of association. It apprehends the several details of the psychical data equally and prepares the way for us to work our way among them later. Free-floating attention provides, so to speak, a storeroom of impressions, from which later knowledge will suddenly emerge. It also creates the prerequisite conditions for those surprising results that appear in analysis as the product of a prolonged unconscious condensation and disassociation of impressions. (Reik, 1948, p. 172) 
Although Reik drew his insights from professional psychological analysis, he wrote Iistening with the Third Ear for the lay person. The same use of attention should also be valid for everyday empathic interaction.

Perhaps this shift of attention to internal space is only difficult to the extent that one has learned behavior which strains attention toward the object perceived. Release of that effort could free more attention for holonomic empathic perception.

The holonomic model adds dimensions to the exploration of empathic perception. It offers an explanation of the core of empathic process, how one human being can attune to and reverberate to another's experience. The model also raises specific issues related to effective use of attention in empathic perception. The following chapter addresses these issues more completely.

1 Initially, Pribram referred to his theory as the holographic model. Other writers used similar terms: "hologram model" (Pelletier, 1978 ) and "hologramic mind" (Pietsch, 1981). In more recent years Pribram re-named the theory the "holonomic model" to include the brain's capacity for perceiving movement and time, and to eliminate oversimplification of his model. The term holographic brain had led some to erroneously infer that Pribram believed the mind performed global holograms, that a reference beam was necessary for perception, and that gross EEG recorded from the scalp were the same as slow wave potentials. The term holonomic was chosen to take his theory beyond earlier formulations of the holographic hypothesis and to extend the scope of computability while still conveying that it is holistic. This term will be formalized in his forthcoming text. (Pribram, 1989a, Lecture $2, p .8)$

2 These lectures are from an unpublished manuscript. The completed text will be published June 1, 1991: Brain and Perception: Holonomy and Structure in Eigural Processing. New York: Erlbaum.

3 Pribram is now pursuing this project at Radford University in Virginia where, after retirement from Stanford, he and several 
scientists are pursuing work on human perception. While waiting for a new laboratory being built to his specifications, they are working with humans and electro-encephalograms recordings to determine if gross measurements that predict choices between colors and shapes are emanating from local or distributed sources (Personal communication, $L$. Gebrant, scientist and former student of Pribram. April 3, 1991).

4 These affects are more fully described as: 1 . Interestexcitement. The eyes track an object, the brow is furrowed and breathing accelerates. This is the response to stimuli entering attention at optimal speed and quantity. 2. contentment-ioy. As the intensity of stimulation decreases we tend to relax. A sudden decrease produces joy; a milder decrease, contentment. In other words, enjoyment occurs in the troughs between waves of demanding experience (like writing a thesis--such joy I look forward to!). 3. Surprise-startle. Our eyebrows rise, we blink, and breathing is suspended. (Eureka! is surprise-the rapid emergence of an idea.) The system is thus cleared so that we might assess the unexected development. 4. Distress-anguish. When we are subject to constant, moderately high stimuli, we feel physical discomfort. Tears may well up. We might also cry out. (Nathanson notes that the voice is a major instrument of the affective system.) 5. Fear-Terror. The eyes are held open in a fixed stare or dart from side to side. The erector pili muscles contract, making the hair stand on end. Altered secretions in the digestive tract produce cotton-mouth, diarrhea or nausea. Blood vessels constrict, leading to cold, sweaty hands. 6. Anger-rage. A very high level of stimulation provides a frown and clenched jaw. The neck is reddened. "Chronically angry people always look muscular, " Nathanson said. "They're living in isometric tension." 7. Dissmell. The response to a bad odor. The upper lip is wrinkled, the nose turns up. 8. The response to a bad taste. The lower lip is protruded as if to spit out the offending substance, and the head juts forward. 9. Shame. Our face feels hot. We tend to turn away, and we experience discomfort. Shame is an impediment to positive affects like interest or contentment. There is a loss of muscle tone in the neck and upper back "When we say a ballplayer is in a slump, we are describing his literal posture." (Nathanson, cited in Brain/Mind Bulletin. p. 6.) 
CHAPTER IV

PATANJALI'S YOGA SUTRAS AND EMPATHY

\section{BACKGROUND}

Yoga is the Sanskrit word for union. It comes from the root yuj, "to unite." The Yoga Sutras instruct one in how to still the mind long enough to perceive directly, and maps a continuum from direct perception of outer objects to direct perception of the subtler regions of inner awareness. It is consistent with the aims of this thesis to explore yoga because the goal of empathic perception is union, the merging of one person's awareness with another's experience.

Patanjali borrowed his concept of perceptual attunement from the Vedas. The Rg-veda was the first to identify cosmic laws and relate them to bio-physics.

Here we find consciousness, cognition, intelligence, reason, discrimination and conscience (the self-regulating moral force) all enunciated as different modes of the same cosmic energy. . . . These ancient thinkers . . have already analyzed the various characteristics of the human mind, coordinated them, and also suggested a viable method for attuning the same to the cosmic rhythm. Patanjali codified this and prescribed mental disciplines for the same. (S. Ghosh, personal communication, 1986)

The Sutras are thus an ancient (true age unknown), classical, Eastern text which gives a precise and detailed description of the function and use of the mind. It is especially suited to western temperament because of its straight-forward approach. Originally, Patanjali formulated the Sutras for verbal transmission. Each dictum was honed into a succinct sutra suitable for memorization. Consequently, the ideas are direct and without embellishment. In a lengthy, explanatory foreword to Aranya's 
(1983) interpretation of the Sutras, Gopalananda characterizes

Patanjali's approach:

The practical side of Patanjali is also reflected in his literary style. Unlike most classical Indian scriptures, the Yoga Sutras do not have a notable style, and they have no particular rhythm or meter. Neither does Patanjali give lofty and romantic descriptions of exalted states. The Yoga sutras are written more like a cookbook than like poetry; they say, do this and we will get such and such an experience; put this and this together and we get that. (p. viii)

Gopalananda explains why the Sutras are adapted to the Western mind:

The value of studying the Yoga Sutras is that they begin, in a very practical way, to open us to this vastly larger possibility of human functioning. Patanjali's analytical and scientific approach is, moreover, extremely palatable to the Western mind. (p. viii)

The entire work consists of 195 aphorisms divided into four books. Book one explains the theory of yoga, book two the practice, book three the internal discipline and byproducts of effort, and book four the transformations that can be achieved.

The ultimate purpose of yoga is to navigate with disciplined attention and understanding through the many modifications of the mind until one can, at will, merge awareness with the most subtle and pervasive aspects of consciousness. The holonomic equivalent might be the attainment of global attention in the implicate domain. Along the way, one gains specific capacities for attention and focus. Included in the text are instructions for attunement to other human beings and discriminative knowledge in use of consciousness for direct perception. It is these insights, nested within the larger context of self knowledge and transcendence, that are germane to this thesis. Therefore, the 
study is selective, with emphasis on material that enhances understanding of empathic perception.

The Vedas expound a matrix for perception that appears to be similar to that of the holonomic brain theory. However, the terms are different. Sanskrit provides more semantic discrimination in these areas than does English. There are no true English equivalents. Where we use the English term "vibration," Sanskrit produces three terms describing the gunas--raja, tomas, and sattva--which represent fundamental aspects of the process of vibration. Raja refers to the principle of motion that is nonrhythmic, tomas to the principle of inertia which involves relative position, and sattva to the principle of harmony and unification of opposites. Sometimes sattva is called vibration because it is the principle of harmony which combines mobility and inertia (Taimni, 1986).
We see, therefore, that all properties can be reduced to their simplest elements--wave motion... action... . and position. . at least as far as the physical universe is concerned and since these are also the ideas associated with the nature of the three Gunas, science has to a certain extent corroborated the theory of Gunas. (p. 175)

As the harmonious combination of rajas and tomas, sattva is not anything apart from the other two. Raja and tomas, of themselves are opposites which do not combine except through the principle of sattva (p. 176). The principles represented by the gunas are explained to be present in varying combinations in all matter and all process. They underlie the principle of triplicity $(96-97)$.

The word "sound" is used to represent the creative function of vibration. Sound, riding on ether, is the source of created form 
(Prabhupada, 1974, p. 108). This emphasis differs from the emphasis of holonomic perception which is based primarily upon light to explain process but which also includes all those bands of the electromagnetic spectrum that are accessible to the human senses.

\section{SELECTED SUTRAS THAT RELATE TO EMPATHIC PERCEPTION}

\section{Mental Modifications and Anisomorphism}

A major premise of Book One echoes the earlier explanation of anisomorphism as everyday conscious, that the essential perceptual processes are disrupted by disturbances in the mind-field. Perception is disrupted by these disturbances, but this can be corrected.

$$
\begin{aligned}
& \text { Yogas Citta-vrtti-nirodhah. (I) } 2 \\
& \text { Yoga is the control of the modifications of the mind-field. } \\
& \text { (Ghosh, } 1980, \text { p. 197) } \\
& \text { Yoga is the inhibition of the modifications of the mind. (Taimni, } \\
& 1986, \text { p. 6) }
\end{aligned}
$$

Patanjali presumes that in most individuals the mind-field does not exist in its optimum state. Instead, it is immersed in perturbations because the mind itself contains perturbations in processing. To perceive accurately, control is necessary. This does not mean control of incoming sensory data so much as it means consciously using the perceptual capacities without distortion, so that the sensory data is incorporated without distortion, and that, conversely, sensory data does not disrupt or distort the mirror (isomorphism) of the mind.

What is an undisturbed mind-field? Ideally, according to Patanjali, one takes the position of the seer who is not identified with the array of interplay witnessed, even while one's attention is wholly 
immersed in it. When this state prevails, then seer, seeing, and seen merge as one and the object or person or idea perceived emerges of itself into the mindfield, without perturbations. If one cannot remain in this quiescent, passive-aware, inner state, then, according to Patanjali, the mind experiences propensities, or distortions, nonpainful and painful (I.5).

Five "propensities" of the mind are explained as nonpainful and painful. Nonpainful proofs translate to reliable knowledge, and there are three kinds: direct perception, inference, and the words of those who have direct knowledge. Only the first rewards the perceiver with results of his own direct perception. The other two give him reliable knowledge, but through either deductive and inductive reasoning or second-hand information from someone else who is able to perceive first hand.

The remaining "painful" propensities result in four kinds of false knowledge: (1) indeterminacy or false notions caused by seeing what one wants or expects to see, (2) conceptual knowledge which is based on words alone, (3) inadequate primary impressions, and, (4) reliance on memory rather than direct perception (I.6-11).

Each of these creates a "modification" or perceptual template which can interfere with viable, direct perception, and therefore interfere with empathic process. An easier way to think of these painful propensities is to recognize them as the result of four aberrant mindstates which do not permit direct perception. They can be loosely 
interpreted as fantasy (projection of expectated forms),

conceptualizing, sleep (dullness while awake), and memory.

Optimally, say the Sutras, these should be overcome, because "with propensities thus destroyed, the mind attains perfect clarity--like a pure crystal that reflects the reflectable" (Sutra 1:41). Shyam Ghosh (1980) explains this sutra:

The mind is then like a crystal that truly reflects the objects placed before it. Like the crystal, again, it is merely the acceptor (reflector) of all that is acceptable (reflectable) through the process of accepting (reflecting). In other words, the seer, the seen and the seeing--all come within the compass of the mind's newly-acquired power. The mind thus becomes aware of its true nature, viz. its double role of "seeing" the "seen" (objects) on one hand, and the real "seer" (Self) on the other. All that is possible, however, only after the propensities have quiesced and the light of intelligence burst forth in its brightest form. (pp. 216-217)

This description of samadhi appears to entail parallels to the description of isomophism via patterned synapses (the seen, the reflectable/acceptable), in computational space between the dendrites of neurons (the organ of seeing which is doing the reflecting/accepting) experienced by awareness in the space-state (the seer who is the reflector/acceptor). When they come together without distortion, samadhi lor harmony, sattva, isomorphism, accurate and direct perception--all approximate parallels) is possible. However, although isomorphic perception, or samadhi, is the optimal state of human perception, it is not the norm. Mental modifications (which appear to parallel anisomorphic consciousness in the holonomic model) impedes the process. 


\section{Four Ealse Mindstates}

Patanjali's description of aberrant mental states will be examined closely because they inhibit direct perception and limit empathic experience. The holonomic parallel to three of the four false mindstates-false impressions, words, and memory--is found in Pribram's explanation of inhibition of incoming vibratory data in the form of internal holophrases in the distributed domain of memory. The remaining false mindstate--sleep--is an aberration of attention. All four result in anisomorphic perception.

Three separate interpretations of each sutra are presented because the differences between Sanskrit and English are great and translations necessarily reflect each author's' personal guidelines. A comparison of interpretations will help offset distortion in any one author's insights. The Sanskrit sutra will be given first, followed by translations from three authors. The study begins with false impressions.

Indeterminacy or false impressions.

Viparyayo mithyă-jñänam atad-rüpa-pratişhtham. (I) 8 .

Viparyaya or illusion is false knowledge formed of a thing as other than what it is. (I) 8 . (Aranya, 1983, p. 27)

The same sutra is interpreted by Shyam Ghosh (1980) more simply: Indeterminacy is getting stuck in a state of false notion. (I) 8 . (p. 201)

Taimni (1986) writes it thus:

Wrong knowledge is a false conception of a thing whose real form does not correspond to such a mistaken conception.

(I) 8 . (P 17) 
A false impression is often the result of wishful thinking or projection of expectation. For example, a man sees a woman smile at him and imagines she wants to sleep with him. Or an elderly woman answers the telephone and is taken in by a salesman whom she feels has her best interests at heart. Empathic perception is not possible when layered over by fantasy or expectation. In Empathy: Its Nature and Uses Katz (1963) relates that research in social psychology reveals that errors in empathy are made by individuals who tend to project (p. 21). Aranya (1986) states that "In reality until imaginary cognition disappears from the thought process, real Rta or realized truth cannot be perceived" ( $p$ 29). Self-aware observation may serve to identify it everywhere. It characterizes much of our dealings with people. For example, humans tend to expect and look for similarities in others (Hastorf, Schneider, Polefka, 1970). This expectation of similarity inhibits the capacity to perceive differences in another person. Miscommunication and the inability to genuinely empathize is the natural consequence. (Bennett, 1979).

In the holonomic model, the propensity discussed above would take the form of inner visualizing that decodes and reprojects anisomorphic holophrases from our neural system, imposing them over incoming sensory data then mistaking them for the real thing. Sensitive self awareness can detect these overlays as overlays and discern the disparity between older, familiar inner holophrases and new, in-forming holophrases. Without enough residual awareness to discriminate between new and old holophrases, humans can become creators and "re-actors" in their own 
isolated show. Direct empathic perception is therefore not possible if this mindstate is predominate

Words:

Sabda-jñānānupātĩ-vastu-sūnyo vikalpạ̣. $\quad$ (I) 9 .

The modification called "vikalpa" is based on verbal cognition in regard to a thing which does not exist. (It is a kind of useful knowledge arising out of the meaning of a word but having no corresponding reality). (I) 9 (Aranya, 1983, p. 28)

Shyam Ghosh (1980) interprets the sutra more simply:

Going by the sound of words only, without the reality, is Delusion. (I) 9 . (p. 201)

The same passage by Tamni (1986):

An image conjured up by words without any substance behind it is fancy. (I) 9 . (p 17)

Here false knowing takes the form of conceptual knowledge or understanding based on words themselves rather than direct monitoring of ongoing process. In the Holonomic model, words are, to use Pribram's (1983a) terms, "cognitive commodities" or holonomic constructs for meaning that cannot match up to the subtlety, novelty and uniqueness of original events. As W. H. Auden (1976) expresses it in his poem "Kairos and Logos:"

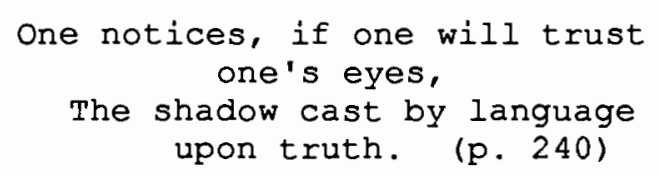

Although words are indispensable in the unfolded aspect of the model, they have little to do with direct, nonverbal perception except to inhibit it or capture it after the fact in cruder form. Empathic 
perception, as it has been defined in this thesis, is facilitated when concepts are abandoned.

When we empathize we are not confined to using the stock of labels or descriptive words at our command. Through our feelings, we sense more of the quality of the feelings of others. Even if we cannot give a name to what the other person is experiencing, to a degree we can experience it ourselves and appreciate it more realistically and accurately. . . . In the appreciation of the complexity and mystery of the human personality, there are degrees of concreteness and reality of understanding. When the knower employs his own feeling and does not rely exclusively on concepts that are necessarily abstract and stereotyped, he understands more profoundly. (Katz, 1963, pp. 16-17)

Aranya (1983) parallels Katz' comment in this statement about the nature of perception:

When, however, the memory (1) of the conventional meaning of the words disappears, the knowledge gained through Samadhi becomes free of Vikalpa contained in the ideas formed through verbal instruction or inference. The true nature of the object contemplated upon is then revealed and this state is called Nirvitarka Samapatti or engrossment free from verbal thinking. It is the truest perception and is the root of inference and testimony which are derived from it. Sutra (I) 43 . (P. 95)

He is unequivocal in his indictment of words as impediments to perception.

In reality, until imaginary cognitions disappear from the thought process, real Rta or realized truth cannot be perceived... . So long as we go on thinking with the help of words, the Vikalpa or vague cognition will continue. (Aranya, p 29)

Pribram (1983a) makes a distinction similar to that of Patanjali

between direct phenomenal experience and the arbitrary nature of perception that is affected by word constructs. He describes words as cognitive commodities in the enfolded order of memory that can affect the re-presentation (unfolding) of primary experience: 
The brain scientist, on the basis of considerable evidence, notes that cognitions enter into perceptions by way of preprocessing: The parts of the brain involved in knowing actively alter the functions of the sensory projections at several stages.

Despite this evidence of cognitive preprocessing in sensory channels, there is a hierarchy that can be made out in our perceptual-cognitive processing. There is, first, a level of "complementation" in which images of objects are present in experience. This presentational level appears to be directly related to phenomenal experience. At the other extreme is the linguistic level, which is obviously representational and in which the connection between representation and what is re-presented is largely arbitrary. (pp. 33-34)

Words are conceptual holophrases incorporated in the top-down

functioning of our nervous system. Words create arbitrary figure/ground choices for attention that in turn, can restrict ongoing processes of direct perception.

Words may be useful if their limitations are understood. Words may be helpful when they raise questions or evoke the unknown as well as the known. Words may be necessary after empathic perception to explicate what has been perceived. Carl Rogers (1975) explains, "It seems to me that only when a gut level experience is fully accepted, and accurately labeled in awareness, can it be completed" (p. 8). However, the core process of direct empathic knowing is necessarily nonverbal. Reik (1948) adds, "It is not at all difficult to find words for what we think. It is much more difficult to find out what we think" (p. 208 ). It would appear then, that Patanjali, Pribram (1983a) and Reik (1948) all recognize words as constructs which can modify or inhibit direct perception. When one responds to new events and interactions through the filter of neurological constructs based upon words, the freshness of primary experience is diminished. Verbal concepts reroute 
attention to well-tracked neural engrams and reinforce them. "Labeling results in reacting to the label and its connotations rather than to the experience itself. This process may be inversely proportional to the degree of mindfulness" (Deutch \& Deutsch, 1963, p. 21).

Because humans incorporate language into perception from birth and reinforce it, continuously and unconsciously, in daily communication, this mental modification may be more difficult to approach and correct than false impressions. The arbitrary nature of words as constructs is often not understood until other languages and cultural constructs are encountered (Condon \& Yousef, 1975; Hall, 1959, 1976; Whorf, 1956). Western culture, particularly, imbues a fondness for words (Hall, 1976). The separation of noun from verb in English could in itself diminish the experience of empathic perception and have impact on the way Westerners experience others. When the noun ("I") is experienced as separate from the verb (what "I" do) and object (the other person), an insensitivity may ensue. One's welfare is no longer understood to be directly interrelated with that of the "object.". The perceptual template, or in Pribram's (1983a) term the "cognitive commodities," can be seen to be a serious modification of direct mental processes.

The holonomic model is helpful for understanding Patanjali's specific and seemingly arbitrary choices of mental modifications. One can visualize the cause of false mindstates as inner holophrases which do not correspond with sensory data. The mind's predilection for choosing the more familiar internal pattern may be the line of least resistence. It may be easier to fall back on existing holophrases than 
to invest new awareness to incorporate new ones. In the case of false notions (Sutra I.8), the pattern is chosen because the person prefers to feel or think in that way and does not take into account the alternative information, even though the false notion usually cannot be verified with the senses and the genuine perception can. In the case of words (Sutra I.9), however, the choice is largely determined by cultural context and habit. In both cases perception is at variance with isomorphism in synaptic space. Patanjali identifies false mindstates by their painful and imperfect result; the holonomic brain model enables them to be identified and even visualized as inhibitors of ongoing process.

\section{Sleep:}

Abhāva-pratyayālambanā vẹttir nidrā. $\quad$ (I) 10 .

Dreamless sleep is the mental modification produced by condition of inertia as the state of vacuity of negation. (I) 10. (Aranya, 1983, p.30)

The propensity of Dormancy harbors a sense of deficiency. (I) 10 (Ghosh, 1980, p. 201)

That modification of the mind which is based on the absence of any content in it is sleep. (I) 10. (Taimni, 1986, p. 18)

The third false state of perception is the torpid, tomasic state called "sleep." Dullness or thickness might make a good English equivalent. This term applies to the quality of awareness rather than the personality. It is does not refer to sleep, per se, in the English language. This mental modification refers to awareness that is too deficient to take in a faithful perception. Dormancy (nidra) is a propensity that is born of the quality of inertia (tomas) in an 
excessive measure. During dormancy "the mind temporarily withdraws and dissociates itself from external objects of interest" (Ghosh, 1980, p. 201). When this happens habit and inner constructs become predominant patterns for behavior.

The state of sleep is a deceptive one. The person may be physically awake, moving, talking, but on rote, stuck in habit, or riding the energy of erratic mood states such as anger or fear with no one "minding the store" of incoming impressions. Thinking in terms of the holonomic model, one could recommend therapy. Shock could catapult one out of this state by significantly raising novelty to a new threshold, overriding the habit state to create awareness (see principle of awareness via dishabituation, Chapter III). So could the removal of familiar habits through travel, unexpected events, or loss of familiar routines. De-automization can also be achieved by actively monitoring inner state space through sustained attention--the purpose behind practice of zen exercises. According to Gill and Brenman (1959): De-automatization is an undoing of automatizations of the apparatuses--both means and goal structures--directed toward the environment. De-automization is, as it were, a shake-up which can be followed by an advance or retreat in the level of organization [of attentional state space] . . . Some manipulation of the attention directed toward the functioning of an apparatus is necessary if it is to be deauomatized. (p. 178)

One can invest awareness on the familiar with such habitual, fierce intensity, that often no one is at home--self aware--in the arena of synaptic patterned activity. This may be why Aranya (1983) offers this practical advice: "To stop the mental modification due to sleep, the first thing to be practised is constant calmness of the body" 
(p. 31).

The prevalence of sleep as a mental modification may become more apparent as one gains control of mindstates. With the capacity for r sustained awareness, one might perceive others acting as if they are in a dreamstate, not truly communicating with one another. The characters in the French Theater of the Absurd--i.e. Waiting for Godot (Beckett, 1954)--provide cartoon dramatics of incomplete and inaccurate perception, the lack of genuine communication, and the inability to listen and empathize when the state of sleep is predominant. Calmness might free the attention and enhance residual awareness, enabling one to bring together the witnessing awareness of the seer with the act of seeing the seen.

The holonomic equivalent of this state would be the absence of witnessing self-awareness, with incoming perceptions disconnected from a mind that is no longer choosing to attend them, electing, through default, to act from habitual inner patterns instead.

\section{Memory:}

Anubhūta-viṣayãsạ̣pramoṣạ̣ smrtih. (I) 11 .

Recollection is mental modification caused by reproduction of the previous impression of an object without adding anything from other sources. (I) 11. (Aranya, 1983, p. 32)

Ghosh is more concise:

The collection of impressions of perceived objects is Memory. (I) 11. (1980, P. 202) 
Finally, Taimni (1986) describes memory in a curious manner, suggesting, perhaps, that one should experience things and events more as process than form:

Memory is not allowing an object which has been experienced to escape. (I) 11 . (P. 20).

Recognizing memory as a false mindstate forces one into the present. Direct perceptions happen in the now, in the primary holonomic domain. When one responds to another as they were and not as they are, empathic perception is not possible.

This false mindstate may have an additional cost. The nonempathic person, seeing another as they were rather than as they are, may not participate fully in that person's growth or change. The nonempathic person cannot reflect back new and emerging qualities in the other person. Reik (1948) begins to discriminate between active awareness and memory saying, "it would have been more to the point, more advantageous, to treat the question of attention separately, and not to confuse it with that of memory" (pp. 159-160).

Memory arises out of enfolded impressions. These were originally the result of direct perception, misapprehension, incomplete impressions, or even former memory. Memory can be a false mindstate even if the memory is accurate, however, because memory cannot substitute for present process. This may be what Taimni was expressing in his interpretation. All false mindstates are related in that they cause attentional neglect of the ongoing holonomic event. In commentary, Ghosh (1980) relates how memory, indeterminacy and sleep can compound. 
All objects perceived by the senses leave their impressions upon the mind. These, after they have duly furnished the "proof" of their existence, are collected and stored there for future use. When the mind RE-cognizes them and REcollects them, they RE-appear from the storehouse called Memory. . . Indeterminacy and Delusion, lie inhibited or dormant in the mind during the waking state. In other words, they remain stored in memory--in the unconscious part of it--during the period of Dormancy (sleep). (p. 202)

Patanjali's Sutras go on to describe the lifestyle, energy, emotional and mental states that contribute to the ability to perceive directly. He gives eight steps for achieving direct perception. The final three steps together are called samyama, and they result in samadhi.

He describes a continuum of direct perception from inanimate objects, to human interaction, to the nature of the mind itself--a progression of attention that parallels the continuum of holonomic constructionist reality from the unfolded, explicit domain to the lensless enfolded, implicate domain. He observes that as one becomes adept at managing attention and understanding the potentials of the mind, new capacities are developed. These are discussed in Book III). They are natural abilities that could appear supernatural in a dualistic construct, but some may be understood as normal abilities in the holonomic paradigm. Among these is the ability to know the contents of another man's mind and heart. Before studying these two sutras, this paper will turn to the principle that may be central to both models, the achievement of algebraic isomorphism, to examine Patanjali's understanding of the process in greater depth. 
ISOMORPHISM, SAMADHI AND DIRECT PERCEPTION

The goal expressed in the Sutras is samadhia clear state of mind that resonates accurately to whatever it contemplates. Here it is described again, this time by Taimni and Aranya.

In the case of one whose Citta-Vrttis (modifications of the mind) have been almost annihilated, fusion or entire absorption in one another of the cognizer, cognition and cognized is brought about as in the case of a transparent jewel (resting on a coloured surface). (I) 41 . (Taimni, 1986, p. 96)

When the fluctuations of the mind are weakened the mind appears to take on the feature of the object of meditation-whether it be the cogniser (Grahita), the instrument of cognition (Grahana) or the object cognised (Grahya)--as does a transparent jewel. (I) 41 . (Aranya, 1983, p. 89)

Patanjali's approach has been to achieve the goal by eliminating the impediments thereby allowing the result--isomorphic perception--to follow of itself. The sutra does not state that perfect isomorphism is required to attain direct perception. Rather, it affirms that when one "almost annihilates" modifications of the mind or "weakens" the fluctuations of the mind, the transparent state of mind follows naturally, without any doing. Therefore, Patanjali observes that isomorphism is accomplished even when the process is imperfectly practiced. This would suggest that the natural perceptual state is isomorphic. Consequently, a valid method for achieving it is to eliminate the obstacles and allow the natural result to follow. This concept may not be as acceptable to Western minds, which places emphasis on activity over passivity. Patanjali's explanation of the underlying principle, given below, may help one shift into an alternate view and 
answer the question, "How is it possible to access the inner nature of an object or person by merging the mind with it?"

Keeping the explanation of the Gunas and their relationship to one another in mind, the following principle of triplicity underlies his method:

The manifested Universe is not really a duality but a triplicity and that is how every manifestation of reality at any level or in any sphere has three aspects. These three aspects corresponding to the subjective and objective sides of manifestation and the relation which must exist between them are referred to in the present Sutra as Gahitr, Grahana and Grahya and may be translated into English by sets of words such as knower, knowing, known or cognizer, cognition, cognized or perceiver, perception, perceived.

This fundamental fact underlying manifestation, that the one has become the three, is the basis of the mysterious identity which exists between these three apparently different components of the triplicity. It is because the one reality has become the three that it is possible to bring about a fusion of the three into one, and it is this kind of fusion which is the essential technique and secret of Samadhi. (Taimni, 1986, pp. 96-97)

These three aspects correspond to the three aspects of holonomic perception described in Chapter III of this thesis--the seen, the organ of perception, and the seer. Just as Pribram describes a three-fold relationship of transforming, unfolding, and enfolding as fundamental to constructionist reality, Patanjali also iterates that three aspects interact to result in a subjective and objective manifestation. Whereas Pribram's Western emphasis is upon the capacity of the transform principle to explain three domains, Patanjali's Eastern emphasis is upon the capacity of human attention to enfold them. In Samadhi, the three become one, and when it does, Patanjali asserts that direct knowing is achieved. Taimni (1986) looks more closely at the experiential aspects of this reverse process in the following explanation: 
The question as to how it is possible to know the innermost nature of an object of meditation by fusing the mind with it is a very interesting one and has been dealt with fully in considering [sutra] I.41. It will suffice to point out here that the apparent disappearance of self-awareness means really dissolution of the subject-object relationship and their fusion in consciousness. With the disappearance... . a faculty higher than the intellect comes into play, and the perception of the reality hidden behind the object takes place through the instrumentality of this faculty which perceives by becoming one with the object of perception. The perceiver, the object of perception and perception become fused in one state. (Taimni, 1986, p. 283)

Samadhi is considered by Patajali to be a desired state worth achieving because the process it embodies can be used to understand a wide range of perceptions:

When the power of Samadhi is acquired by the mind, one can be wholly absorbed in any object of the category of Grahya (knowable, i.e. phenomenal objects comprehensible by the senses), Grahana (internal and external organs) and Grahita (the receiver, the empirical self). (Gopalananda, cited in Aranya, 1981, p. xxii)

The three terms listed above--isomorphism, samadhi, and direct knowing appear to be differing explanations of the same process. Just as algebraic isomorphism is central to the face-up, face-down multidimensional perceptional potentials inherent in the holonomic brain model, the practice of samadhi is central to efficacy in the human perceptual potentials described in Patanjali's Yoga Sutras, opening up a range of direct knowing. Pribram's contribution is to scientifically and mathematically map the process; Patanjali's contribution is to experientially describe methods that develop the human perceptual potentials that Pribram's model describes.

Isomorphism is possible because of algebraic transforms of vibratory patterns in synaptic space; samadhi is made possible by 
practices and disciplines that enable attention, synaptic space-state, and vibratory information to become coherent, and work essentially as one. This may be the prerequisite condition so that algebraic transforms happen and isomorphism is experienced. If so, then West and East models merge here, in a vital and enriching way.

It would seem also that from both perspectives, the models are useful for understanding and maximizing human perceptual potential. In the holonomic model perception is isomorphic when the transforms in the active, primary domain result in faithful re-representations and distributed memory of primary experience. In Patanjali's model the goal is union through samadhi, which may be a state of isomorphic perception in which seer, seen and the object or state perceived are as one with no intervening variables or mental modifications. Primary experience (Pribram) or direct knowing (Patanjali) is explained to be the result in either case (see figures 5 and 6 ). It is also the goal of empathy to directly perceive the other person as they are, without intervening mental aberrations.

However, Patanjali takes one farther into use of process than does the holonomic model. Books I and II of the Sutras concentrate first on understanding the optimal perceptual state and the mental modifications that obstruct it. Then, Book III lists techniques for using the process to gain specific kinds of knowledge and abilities. Two of these sutras may be applied to empathic capacity. Patanjali gives two attentional methods for gaining accurate knowledge of another, by focusing on one's 
TRANSFORMS IN

SYNAPTIC SPACES

"What the brain is doing"

SEEING

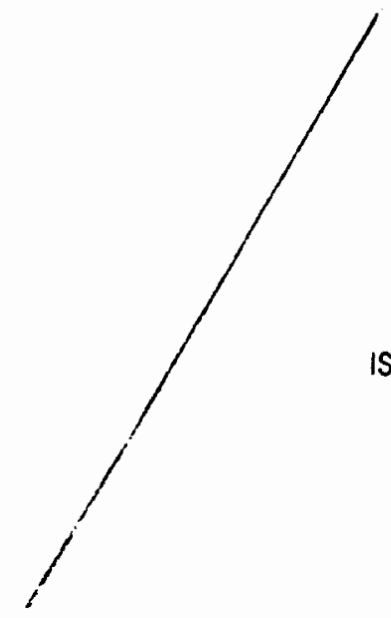

ISOMORPHISM
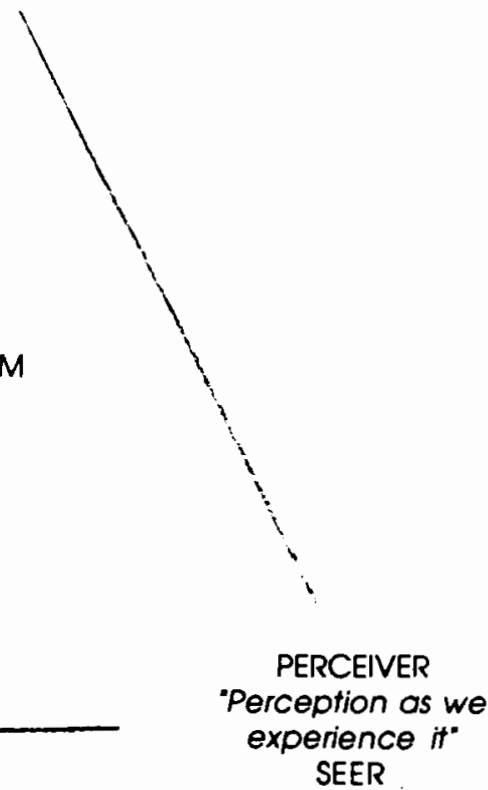

SEER
PERSON/OBJECT/FORM

Thot which is being seen"

SEEN

Eigure 5. Isomorphism="same form." A graph of the three essential elements necessary for algebraic isomorphism based upon Pribram's explanation (1989b). "Same form" in constructional realism refers to faithful representation of forms perceived. 


\section{PERCEVING}

COGNITON

KNOWING
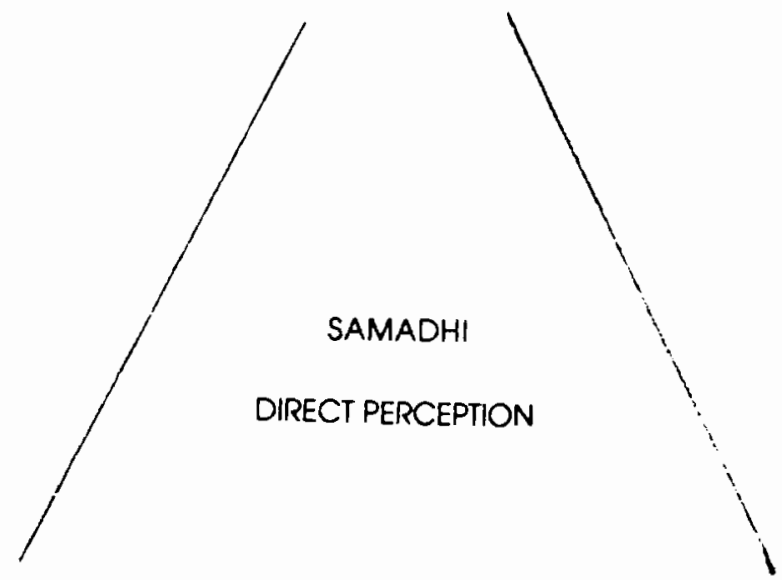

PERCEIVED

KNOWER

Eigure 6. Samadhi=direct perception. A graph based upon Patanjali's principle of triplicity which is explained to include three elements which must become fused (as one) through elimination of mental modifications in order that samadhi or direct perception may be achieved. It contains essential parallels to the three interacting components of algebraic isomorphism in figure 5. 
own heart while attuning to another person, and by focusing on "notions:"

Hẹday citta-samvit. (III) 35. (Taimni, 1986, p. 335)

(By practising samyama) on the heart, knowledge of the mind is acquired. (3) 35 . (Aranya, 1981, p 309)

(Samyama) on the heart brings knowledge of all minds. (III) 35. (Gosh, 1980, p. 262)

(By performing Samyama) on the heart, awareness of the nature of the mind. (III) 35. (Taimni, 1986, P. 335)

Ghosh (1980) explains that Hridai (heart) refers here to the conscience which is supposed to be centered close to the heart, has the quality of purity (sattva) and is also a center of energy through which

consciousness functions. It is ever available as the inner voice that must speak the truth despite man's intellectual conversion to the contrary. He says that when one practices sanyama--meditation that culminates in samadhi--on this conscience (heart), "everything in his own as well as in that of another mind becomes crystal clear to him" (p. 263).

Taimni (1986) comments:

In this sutra. . . the method of unfolding intuitional consciousness is given. As the intuitional consciousness transcends mental consciousness the first step, naturally, is to acquire true knowledge concerning the nature of the mind and how it modifies pure consciousness, i.e. consciousness of the Purusa. This knowledge is gained by performing Samyama on the heart. . . . As Citta (mind) is merely the product of the interaction of consciousness and matter (1-2) it should be easy to see why the common centre of all the vehicles through which consciousness functions (the heart) should also be the seat of Citta. The senses are merely the outposts of Citta and should be considered as part of Citta. (p. 336) 
Reik (1948) also speaks of listening to the heart. He titles an entire chapter "Ask Your Heart What It Doth Know."

A little more or a little less inner sincerity, a small plus or minus of moral courage, is what decides whether we understand ourselves and others. .

If the analyst listens to what others say--and listens even to what they do not say--he will get the messages which, if deciphered, lead to psychological insights that cannot be reached by any other means... . He must break up the ground, dig deeper and deeper into himself, until he reaches the source of all psychological understanding that is in himself. . . I believe that the best advice... . was given more than three hundred years before Freud. Here are the lines that a figure of Shakespeare speaks . . . "Go to your bosom;

know there, and ask your heart what it doth know--" After all teaching is said and all study is done, do not expect answers from your intelligence, from your "reason." (pp, 67-68)

Reik (1948) also quotes Friedrich Schiller's statement, "If thou wouldst understand others, look into thine own heart!" (cited in Katz, 1963, p. 45) .

This sutra may also apply to Reik's (1948) description of empathic reverberation. Through self-understanding (directly perceived), something familiar to us is evoked by empathic interaction with the experience of another. In the holonomic model this "in-formation" could correlate directly, nonverbally, and nonlinearly with the vast depository of memory in the transform domain. One's encoded experience would interact with the incoming holophrases of the other. These correlations could form the basis for reverberation that is compatible with Patanjali's choice of the heart as focus.

While Western reference to the human heart is often sentimental, Patanjali's instruction to look into the heart is not. Through samyama that culminates in samadhi, awareness must go beyond human emotion to 
create an unmoving internal space-state in which empathic reverberation can occur. A witnessing awareness quietly monitors this space-state without sentiment. The inner witness is essential to the process. Katz (1963) makes this interesting distinction about the experience of reverberation:

Reik described the process as a vibration in two rhythms at the same time, a reverberation which yields insights that appear unpredictably. It is a paradoxical process in which we are at the same time fully absorbed in the identity of the other and yet capable of an experience of ourselves as separate personalities. Part of our self is fused with the identity of the other. Yet another part of our feeling is capable of responding to this experience as the experience as the external thing it is. (Katz, p. 44)

The discipline of samyama to achieve samadhi is a counterpart to the use of inner attention to achieve isomorphism, the prerequisite of accurate holonomic perception. There may be a vast difference between perceiving another as they are, and the perception of the person as we think about them. In the first state of perception, the person practising samyama is stable, quiet and watching as the person perceived emerges into the inner space-state where watcher, space-state, and person perceived are merged as one. In the second state, the person is thinking, moving mentally around the subject, attending first this, then that aspect. The inner space-state may not be quiescent enough for the direct impression to fully emerge.

If the holonomic metaphor is viable, then the first method of perception is necessary to access the enfolded, distributed domain and obtain faithful results. To become more empathic and to accurately perceive others, one might benefit by repeated samyama on his own heart. 
Then, when directly perceiving another, reverberation may be more complete. The insight gained while attuning to one's own heart may deepen the capacity to resonate to the content enfolded there while directly attuning to another person. Thus, Patanjali's instructions may provide a technique which is relevant for the distributed, enfolded order of the holonomic paradigm.

A related sutra explains how to know the contents of another's mind. Here the instruction is similar because one must first put his attention within:

Pratyayasya para-citta-jñ̄anam. (III)19. (Taimni, 1986, p. 317)

(By direct perception through Samyama) of the image occupying the mind, knowledge of the mind of others. (III) 19. (Taimni, 1986, p. 317)

Knowledge of another's mind is gained by concentrating on the apparent. (III) 19. (Ghosh, 1980, p. 256)

(By practicing samyama) on notions, knowledge of other minds is developed. (III) 19. (Aranya, 1986, p. 291)

Aranya (1986) explains that first one has to understand the nature of thoughts in his own mind. He must understand how thoughts arise and subside. Then by making one's mind unqualified by thought and attuning to another's mind, "the thoughts that rise therein they are the thoughts of the other person" (p. 291). He also goes on to say: Thought-readers cannot say how the thought is transferred, but they just feel that the thoughts are not their own. Some can read other people's thought without any effort when the other person is in the process of thinking something. Anything previously felt but since forgotten may also be sometimes known by a thought-reader. (pp. 291-292).

Ghosh (1980) expands the meaning somewhat to emphasize also the physical body : 
Such direct and close study (involving application of the method of samyama) of the physical form of another person-i.e. the physical features of that body--enables the yogi to know the contents of that person's mind. (p. 257).

This explanation incorporates the paradoxical principle of holism, that knowledge of the whole of a person is accessible through understanding of parts. Consequently, "notions" carry meaning and understanding if attended to with awareness.

Again, Theodore Reik's experiential insights echo those of Patanjali:

Unnamed impressions become the means of communicating psychological knowledge. . . . No small power of communication is contained in a glance, a person's bearing, a bodily movement, a special way of breathing. Signals of subterranean motions and impulses are being sent silently to the region of everyday speech, gesture and movement . . . . We are reminded of Freud's view that mortals are not so made as to retain a secret. "Self betrayal oozes from all our pores." (pp. 135, 142-143)

The sutra above would seem to rely on inference from direct perception that accesses both ongoing affective cues about the other person and intuitive responses from reverberation of those cues with enfolded memory.

EMPATHIC PERCEPTION VIA AWARENESS IN A LENSLESS DOMAIN

Reik (1948) describes how to use attention in the "unconscious" (distributed) order to take in and interpret nonverbal messages from the other person. His description parallels Patanjali's instructions on attending to the inner images that occur when one has focused upon another:

The psychoanalyst who hopes to recognize the secret meaning of this almost imperceptible, imponderable language has to sharpen his sensitiveness to it, to increase his readiness 
to receive it. When he wants to decode it, he can do so only by listening sharply inside himself, by becoming aware of the subtle impressions it makes upon him and the fleeting thoughts and emotions it arouses in him. It is most important that he observe with great attention what this language means to him, what its psychological effects upon him are. From these he can arrive at its unconscious motives and meanings, and this conclusion again will not be a conscious thought-process or a logical operation, but an unconscious--I might almost say, instinctive--reaction that takes place within him. Again, The meaning is conveyed to him by a message that might surprise him much like a physical sensation for which he is unprepared and which presents itself suddenly from within his organism. Again, the only way of penetrating into the secret of this language is by looking into oneself. . . . The reader is asked to think this over. A little known and concealed organ in the analyst receives and transmits the secret messages of others before he consciously understands them himself. (p. 147)

Although he may never have read the Sutras, Reik subscribes to a process of attention and attunement that not dissimilar from Patanjali's instructions on direct cognition of objects through samadhi. Reik believed that preparation, self-knowledge and attention were the keys to enabling one person's unconscious to hear messages from another person's unconscious. He called this the development of the "third ear" and comments,

One of the peculiarities of this third ear is that it works two ways. It can catch what other people do not say, but only feel and think; and it can also be turned inward. It can hear voices from within the self that are otherwise not audible because they are drowned out by the noise of our conscious thought processes. (p 146-147)

Put in terms of holonomic empathic awareness, isomorphism--the brain's coherent reflection of another's inner affective states--may be possible if one attunes with sufficient sensitivity so that perceiver, perceived and perception are as one in neural processing. Then the perceiver, via attention, has access to information about the person in both domains, 
the unfolded and, importantly, the enfolded. This description may in itself be an a useful definition for empathic perception.

The process of samadhi to achieve direct perception is not to be confused with identification, according to Patanjali, because even though the perceiver, perceived, and organ of perception can merge as one, the Purusa is the real seer and is never identified with the process. Taimni (1986) comments upon the real seer in (IV) 16:

All our words such as "knowing," "perceiving" are so closely identified with the activities of the mind that a new word is really needed to indicate this peculiar ultimate reaction of the Purusa to all mental processes. But, in the absence of such a word in the English language "witnessing" will perhaps do for the present. ( $p$. 415)

This corresponds to some extent with Pribram's (1987) explanation of attention in the space-state:

Different states organize conscious processes in different configurations much as different languages differently organize what we mean to say. There is always some "hidden observer" who in some sense "knows" what is being configured, what is meant. The "hidden observer" is the "state space" within which the states develop. (p. 5)

Pribram (1987) further explains that "the organization of attention can be determined endogenously by states or exogenously by sensory input, or by both" (p. 7). It would follow, then, that for awareness of ongoing events, both kinds of organization of attention would be present: the active, exogenous aspect of the mind that merges with the object (referred to as the moving kinesthetic sense of self in Chapter III); and the more pervasive, endogenous, passive awareness that witnesses the entire process and is not moved by it. When one changes the emphasis from active kinesthetic attention (which also provides one with a sense of "I"--see "Kinesthesia and a corporeal sense of I") to inner 
awareness, the difference may be more easily discerned. When this happens, the first tendency may be to become unidentified with the active, kinesthetic observer by saying that there is no observer. Goldstein (1986) describes this meditative experience:

Don't be afraid to use the mind in that way, to investigate. But not with words, not with thoughts, not with concepts. Try to get a feel, a sense of the process of consciousness happening together with the object. This experience brings freedom from identification with the observer. Insight comes from the realization that observation is going on without an observer, witnessing without a witness. (p. 62)

However, conscious empathic perception requires the presence of both kinds of awareness, the moving kinesthetic self that changes boundaries to synchronize with and incorporate the other person, and the witness in the inner space-state which watches. Patanjali calls the commonly experienced perceiver the Buddhi, and identifies it as the moving aspect of the material mind which is not self-illuminating (Taimni, 1986, p. 420). The second illuminating aspect is independent of the moving mind, being more pervasive. Patanjali says this is the purer consciousness of the Purusa (p. 420).

Although this distinction seems arbitrary at first, it may make a profound qualitative difference to the act of empathy. Some aspect of attention must completely merge with ongoing experience to achieve isomorphism. There must also be a way to merge awareness without becoming identified. Sheep unconsciously move with one another. They bleat if another sheep is in distress. Examples of empathic attunement are everywhere in the animal and human world; however, most are unconscious behaviors. The awareness emanating from the Purusa--that 
which witnesses the process without becoming a part of the process-alters empathic union by making it a conscious experience.

In addition, attention that is firmly concentrated inward may draw impressions to that inner space-state and experience them more coherently than attention which streams out from the person to identify with all it contacts, attending to first this impression and then that impression. Isomorphic awareness may require effort before it can become sustained and effortless. Drawing from both perspectives-Patanjali and the holonomic model--one might expect that isomorphic awareness is difficult to sustain without the witnessing awareness of the Purusa because the observer can be pulled into the magnetism of the other person's attentional state. Taimni (1986) explains that "the Purusa is aware, in an unbroken manner, of all the changes which are taking place in the mind and it is not possible for any change to escape his notice" (p. 415). Although the terms relating to attention are different, Reik's (1948) long chapters on the nature of attention necessary to empathic awareness describe a quality of listening that is inwardly attuned and sustained, an awareness that seems to parallel Patanjali's descriptions of nonidentified witnessing.

One can find congruency in Pribram's (1971) holonomic brain model and Patanjali's description of false mindstates. Pribram maps the process; Patanjali identifies mental disfunctions that inhibit it. Pribram's model helps explain Patanjali's choices; Patanjali helps to operationalize Pribram's model. In four Sutras, Patanjali identifies the sources of aberrant thought processes. The result of these mental 
modifications is subtle to gross false impressions that cripple our ability to perceive truly.

Patanjali's contribution is useful because it represents a nonWestern cultural perspective. Westerner's are not accustomed to the idea of fantasy, words, inattention, and memory as false thinking. The inclusion of both Eastern and Western perspectives here gives a more rigorous standard against which to measure habitual processes of perception. The choices for attention are concrete and may make discrimination between isomorphic and anisomorphic mental states more apparent. Those who wish to improve empathic awareness can refer to the Sutras for a rigorous standard against which to measure subjectively experienced states. If the perceptual field is free of imagination and expectations, overlays of words, dullness or past memories, then powerful buffers to empathic perception may be eliminated.

An added advantage to this approach is that not doing is somewhat easier than doing. In other words, Patanjali tell us how to get out of our own way by identifying and refusing to indulging in false mindstates. By starving them of attention, awareness is free to experience what is playing directly in the synaptic arena. By default, the more vital and primary event comes into the field of awareness. As a "not that" mental discipline the indeterminate mindstates can be recognized, abandoned and attention can then gravitate to what is happening in the present. In this way, empathic identification, incorporation and reverberation can proceed unimpeded. 
This thesis has explained and combined Western and Eastern models of perception for their relevance to empathic process. Their complementary nature provides a wealth of material for rethinking the empathic process. Pribram (1985b) also anticipates the worth of combined perspectives to review questions about human perception:

Developments in mathematics and computer architecture allow us to model psychological processes as diverse as imaging and intuition, as respectable as sensory psychophysics and as non-sensical (non-sensory) as mystical experience. For a half-century, quantum physicists such as Niels Bohr, Schrodinger, Einstein and Heisenberg shared their insights with us by pointing out the similarity of their findings with those of the Veda and Upanishads and other spiritual disciplines. Is it not time that psychology listens, places the Newtonian cosmology in perspective and comes to grips, where relevant, with the models developed in the 19 th and 20 th century mathematics and physics? (p. 6)

Using the insights gained from Chapters III and IV of this thesis, this study turns again to communication and empathy. 


\title{
CHAPTER V
}

EMPATHY REVISITED

\author{
NEW PARADIGM PERSPECTIVES: GENERAL \\ IMPLICATIONS FOR COMMUNICATION AND EMPATHY
}

The purpose of this chapter is to take into consideration the perspectives explored in earlier chapters and suggest changes in thinking, behavior and theory to account for those perspectives. In general, the field of communication still responds to Newtonian/Cartesian metaphors, and has not yet followed the lead of scientists into the realities of the quantum order to explore implications for human interaction. The new bridge provided by neuroscientists and explained in this thesis makes that paradigm shift possible.

Marshall Mcluhan enthusiasts could point to his thesis that "the medium is the message" (McLuhan \& Fiore, 1967) and draw parallels between our accelerated electronic age and the human equivalent of expanded neurological capabilities in light of the holonomic paradigm. McLuhan (cited in Littlejohn, 1983) comments:

Electric circuitry profoundly involves men with one another. Information pours upon us, instantaneously and continuously. As soon as information is acquired, it is very rapidly replaced by still newer information. Our electrically configured world has forced us to move from the habit of data classification to the mode of pattern recognition. We can no longer build serially, block by block, step-by-step, because the instant communication insures that all factors of the environment and of experience co-exist in a state of active interplay. (p. 267) 
Similarly, the human who holonomically attunes to the vast range of vibratory information, from gross to subtle, within his or her own system, may have access to continuous, direct perception that is largely screened out through ordinary perception. For more effective use of empathic capacities, one can no longer afford to place attention primarily in a secondary sensory reality for information in the form of objects, words, and sequential events which require data classification and information "block building," but rather one can also attend to intuitive recognition of patterns of knowing and feeling that refer themselves as one focuses and cares about them.

This re-empowerment of direct knowing through holonomic awareness may carry with it "at-one-ment" and holistic perception, enabling one to recognize the connectedness of events, persons and objects. Pribram and Patanjali remind us that we exist in a state of active interplay. Separateness is an illusion. We are profoundly involved. We are a channel for the interplay.

Most current communication theory addresses only sensory reality, the unfolded order. With the assumption of two orders of reality and the a mediating, active transform domain which makes isomorphic perception possible, no area of communication theory remains unchanged by the holonomic brain paradigm. Pribram comments in his new text on holonomic brain processes, "Communication can only occur when both frames of reference, both worlds are acknowledged by discovering the tansformations that connect them" (1989a, Lecture 2, p. 26). To properly consider the topic, new theories should be articulated which 
include all domains in constructionist reality and an understanding of the principles and nature of the transform which unites and explains them. Understanding the nature of the transform neccessarily requires knowledge of the role of human perception as a mediating factor. This knowledge should consider the greater perceptual capacities and increased choices made necessary and possible as we participate in the primary domain to interact in two orders: (1) creating and interpreting a secondary manifest reality--the unfolded order--through the five senses working in concert, while (2) also participating in the lensless enfolded domain, which seems somehow influenced by focus, intent, visualization, and intensity of experience.

New theory would have to consider intracommunication--interaction between the the enfolded and unfolded domains--through willful variation and maintaining of attention in active and passive-aware states of consciousness. Biofeedback may facilitate understanding. Symbols-verbal and sensory--might be explored as objects of focus that would help bridge both realities.

New holonomic communication theory would emphasize the mediating role of empathic attunement because of the interrelatedness and interconnectedness of all experience, events and phenomena. Linear time would apply only as a convenience to communication in secondary, unfolded, sensory reality. In the frequency domain it could emanate out as synchronicity or be felt as an event or point, the density of the point determining how the event would radiate out into manifested reality, altering past, present or future. One could explore the 
ability to create such density "points" in the frequency domain through intent or visualization--feedback through inner holonomic abilities. More attention could be placed on awareness and the paramount skill of sustained, focused attention and visualization.

"Re-cognizing" one's inseparability from the whole, one would communicate with increased sensitivity to others and one's environment, experiencing the greater "in-forming" capacities of the neural system-one's own and others--and realizing also that all persons and manifested things participate in some way with indivisible vibratory reality.

Through holonomic attunement we might increase our capacity to resonate to the affective reality of another. This capacity could be of primary importance in an age of rapidly mixing cultures with divergent world constructs. Bennett (1972) explains:

It is convenient to see empathic perception occurring on a level above that of cultural world view and language. Like the other levels, this level also has structure and function. Its structure is not the configuration of world view, but awareness of that configuration. The function of this level is not the direct application of world view into phenomena, but the application of self-awareness to the experiencing of phenomena. (p. 113)

The holonomic model describes constructs as encoded holophrases that interact with and modify incoming experience. Awareness of the same might enable one to be more realistic about the profound differences in the belief systems of others so one would assume difference rather than similarity when interacting with another (Bennett, 1979).

At the same time, holonomic empathic perception gives a viable means for reaching beyond oneself by accessing the ongoing experience of another person more fully. Consequently, one could be both more aware 
of what is not known about the other person, while increasing the capacity for attuning, listening and learning. Listening, itself, would take on new meaning with the realization that the whole is in each part. Intuition would be awakened as one developed methods for sensing more complete essences behind partial representations. In these ways, empathic perception might be viewed as a self-transcending aspect of human experience (Capra, 1982, pp. 285-286).

Ethics, too, might become be more experiential and less proscriptive if we could attune to others with greater sensitivity, thus intuiting their needs and what constitutes harm to them. Communication rules would change as the holonomic paradigm erodes man's sense of separateness which now enables one to ignore or abuse the "other" who is "out there." Competitive behavior would have to take into consideration not only overt communicative acts, but the suggestibility and sensitivity of the frequency domain. One might be be held accountable not only for words and deeds, but inharmonious intent, emotions, thoughts and moods which bring disorder to both domains.

To become truly responsible and effective communicators in the construct of holonomic reality, humans would cease being passive reactors to the manifested world only and, instead, would learn to consciously use their perceptual and creative abilities to interact with and create harmonious realities. Here we would be aided by the examples and teachings of mystical traditions which have been addressing themselves to this dual reality all along. Sufi stories would alert us to the ultimate absurdity of relying upon sensory, unfolded information 
alone. Zen teaching could dishabituate attention and strengthen our awareness of the witness--wake up the "ghost" in the machine--and hone abilities to willfully alter and sustain varied states of attention. Yogic traditions would prepare the body, brain, spine and mind for the neural recircuitry involved as controlled attention shifts awareness from identification with the senses to the entire nervous system and pure frequency domain. Gnostic Christian tradition could strengthen sustained awareness through devotional attitudes--now alien to Western culture--that change emphasis from awareness of the mind to include awareness of the heart as a perceiving instrument. Patanjali's sutras could guide us as we "reduce noise in the channel" by shifting primary attention from the sensory world, purifying our "reference beam"-possible perhaps by harmonizing the body's slow wave potentials and merging seer, seen, and organ of perception--and learning to intuit reality directly from conscious immersion, or immersion of consciousness, in primary vibratory experience.

These new areas of human possibility are appropriately the province of communication theory. They may be intuitively anticipated. The holonomic paradigm reveals and invites exploration into these new domains. Inner personal authority--the empowerment of experiential knowing--takes on new significance as we broaden our communication scope to include both the implicate and explicate, the encoded and decoded domains of reality, through holonomic perception. Human interaction would be enriched with the enhanced understanding of the mediating role of holonomic empathic perception. 


\section{REIK REVISED: APPLYING THE PRINCIPLES OF HOLONOMIC EMAPTHIC PERCEPTION TO EVERYDAY LIEE}

Holonomic empathic communication can be redefined as ongoing transference of direct impressions which occur when perceiver, perception and the person perceived reverberate as one in inner attentional space. The other person is immediately, rather than mentally comprehended, as they are without intervening ideas. The attentional space-state is a paradoxical one of focus, elimination of conceptual boundaries, and dishabituated, sustained awareness. The resulting isomorphic perception accesses the transform domain, encoding holophrases of the person perceived that are then correlated with stored holophrases throughout one's nervous system and later "re-membered." The other person is thus realized empathically.

But what does this mean in everyday terms?

Here Theodore Reik's (1948) four steps deserve re-examination in light of the holonomic metaphor. They hold up surprisingly well to the definition of empathy above. The discussion that follows expands on these steps to include the principles of holonomic perception elaborated in this thesis. Two new prior steps are considered:

1. In keeping with Reik's cryptic names for each step, the first step could be termed "clearing" or "vacating" or even "suspending" (Bennett, 1972). It would be a preparatory process to intentionally clear or suspend one's inner attentional space of judgements, expectations, needs or wants, personal agendas, limiting concepts, past experience with the individual, and so forth. This would minimize 
anisomorphic perception. Three attitudes might help dishabituate preexisting attentional modes and vacate the mind of intervening thoughts, releasing awareness for both an orienting mode toward the other person and an inward shift to attentional space. These attitudes are curiosity, caring and pure, dishabituated awareness that can be sustained. They are mentioned here because their effect might facilitate an unencumbered, open attentional space-state and amplify awareness. Personal emotional states are to be abandoned--even positive ones. Unqualified, calm awareness is the goal so that one can more fully apprehend the other's affective state.

2. Then, to maximize isomorphic awareness, a division of attention is created. However, it is really not a division at all. It can be readily achieved through reawakning the inner, watching awareness in attentional space while focusing ordinary sensory attention on the other person. This state could be called "focus and feeling." The term "focus" would refer to fixation of sensory attention on the other person. This can be accomplished through the kinesthetic, corporeal self described in Chapter III. It would expand boundaries to move toward and to include the other person. In contrast, "feeling" would direct one to sense inwardly and rejoin attention to ongoing, inner awareness--the witnessing state. The center of attentional gravity should be in this inner space. Feeling may seem a contradictory term, and perhaps it could be misunderstood for emotion. However, the intent of this word choice is to shift the perceiver out of a thinking mode into one of direct perception of inner, waiting awareness. The 
division of attention is necessary. Empathic perception is a paradoxical state. As Reik (cited in Katz, 1963) relates:

What is essential in the psychical process going on... is that he can vibrate unconsciously in the rhythm of the other person's impulse and yet be capable of grasping it as something outside himself and comprehending it psychologically, sharing the other's experience and yet remaining above the struggle, au dessus de la melee. (p. 44)

One of the hallmarks of this shift might be abandonment of the sense of time. In the transform domain, time is enfolded. While a portion of attention is focused on details in outer, unfolded reality, the remainder shifts to an inner attentional mode where time, causality and three-dimensional space are not experienced.

3. Identification: Reik's (1948) first step is now the third step. Mimicry, synchronization and internal imitative behaviors enable the perceiver to attune to the other person. These can be both overt and subliminal movements. The outer attention is invested here, similar to the residual, kinesthetically focused attention with malleable boundaries mentioned above. It is ordinarily experienced as self, and here it allows itself to be engaged by the energy fields of the other person. One can listen and talk while attuning to these nonverbal signs, using the situation to synchronize behavior.and attune to the person. Because the inner, watching awareness is the primary and more stable space-state monitoring these behaviors, the whole of attention is not identified with the other person.

Here the explanation differs from Reik who says "Our entire consciousness is projected into the other person that the feelings that inhere in others act upon us" (cited in Katz, 1963, p. 41). If that 
were true, the experience would not be a conscious, self-aware one. Only the ordinary kinesthetic sense of self is projected into the other person. As one is successful in this process, a "blurring" of perception may occur as one's internal space-state allows the incorporation of the affective experience of the other person. One set of inner neurological holophrases gives way to another set. If one wishes to incorporate the other's experience, it is important at this point to allow the ambiguity to proceed and not to interpose an "empathic wall" (Nathanson, cited in Brain/Mind Bulletin, 1991b, p. 7) in the form of resistance through reinforced personal constructs or attentional avoidance of the new experience.

However, one may also decide to consciously interrupt or buffer the process of identification with the other person. As mentioned in Chapter III, empathic interaction is not always desirable or necessary. The core process of empathic understanding is intimate and personal. One enfolds the other into inner neural knowing. The knowledge gained may be as permanent as memory. Although it is an invaluable aid to understanding, empathic interaction can sometimes be unhealthy and unpleasant. For example, the person with whom one interacts may consciously or unconsciously intend psychological harm. The important consideration here is that one be able to use the process consciously. 4. Incorporation: Reik describes this as the act of taking the experience of the other person into ourselves. This is done as the outer consciousness gravitates to and is subsumed by the behaviors, energy levels, affect, intent, tones, and so forth, of the other person. 
Mimicry and synchronizing have enabled the attention to vector toward the unknown components of the other person's psyche. Incorporation would then go past these known aspects toward the unknown invariant holophrases of the other person. These are the targets for the act of incorporation. They are the unknowns that can be realized more fully through empathic attunement. These constitute the mystery of the other person that transfixes one's attention, stabilizing it so incorporation can occur. This combination of synchronization and vectoring make an internal "dance" of attunement toward the unique or "impenetrable" aspects of another. This act carries the process beyond the purview of conscious attention.

5. Reverberation: Reik (cited in Katz, 1963) explains this stage as allowing the other person's reality to commingle with our own:
Part of ourself is fused with the identity of the other, yet another part of our feeling and thinking is capable of responding to this experience. He remains involved, attached, and identified long enough to allow for his personal reactions to be evoked and for deeper levels of his own unconscious to be stimulated. (p. 42)

Here Reik has aptly described holonomic empathic enfolding of information to the transform domain--"deeper levels of his own unconsciousness"--where they are distributed and achieve new correlative capacities--"to be stimulated." He has intuited the passive-active (passive on the part of the doer; active on the part of the "unconscious") nature of the transform domain and knows it is accessible through awareness. One could add that both passivity and faith that the process is occurring, which combined might resemble patience, may be useful attitudes here. One cannot "do" empathy. Doing occurs in the 
unfolded domain; the reverberation process occurs in the enfolded domain where allowing is a more appropriate term.

6. Detachment: To withdraw from subjective involvement and gain the social and psychic distance necessary for objective analysis (Katz, 1963, p. 46). This is a return of the projected kinesthetic self from experience of the other person to the witnessing self. Personal boundaries are re-established. The perceiver returns to the comfort of his habitual sense of perceived self and all that that means. However, one returns knowing that essential aspects of the other person are incorporated into into one's own being, and that the same is there for retrieval later when one experiences "the re-emergence into the consciousnes of the data so assimilated" (Reik, 1948, p. 31), and the cognitive components of empathic process come into play. Reik (1948) comments, "there are no bolts from the blue... . Sudden clarity is preceded by increasing darkness. To speak with Nietzsche: 'That which will kindle the lightning must for a long time be a cloud" (p. 172). One listens within for recognition of insight. More than this, empathic perception has enabled the psyche to transcend itself through awareness of the essence and experience of another human, thus diminishing the limitation and alienation of separateness. 
SOURCES CONSULTED

Aranya, H. (1983). In P. N. Mikerji (trans.), Yoga philosophy of Ratanjali. Albany, NY: State University of New York Press.

Arya, P. U. (Trans., ed.). (1986). Yoga-Sutras of Patanjali with the exposition of Vyasa: A translation and commentary, volume I Honesdale, $P A$ : The Himalayan International Institute of Yoga Science and Philosophy of the U.S.A.

Allport, G. W. (1937). Personality: Apsychological interpretation. New York: Holt.

Aspy, D. N. (1975). Empathy: Let's get the hell on with it. The Counseling Psychelogist, $5(2)$.

Astin, J. S. (1967). Assessment of empathic ability by means of a situational test. Journal of counseling Psychology, 14, 57-60.

Auden, W. H. (1976). In E. E. Mendelson (Ed.), H. H. Auden: Collected peems. New York: Random House.

Bachrach, H. (1976). Empathy: We know what we mean, but what do we measure? Archives of General Psychiatry, 33, 35-38.

Banquet, J. P. Spectral analysis of the EEG in meditation. Electroencephalography and Clinical Neurophysiology, 35, 143-151.

Barrat, E.S. (1970). Empathy: Towards a psychophysiological definition. Diseases of the Nervous System, 31, 464-467.

Bateson, G. (1979). Mind and nature: A necessary unity. New York: Dutton.

Bennett, M. J. (1972). Empathic Perception: The operation of selfawareness in human perception, Unpublished master's thesis, San Francisco state College, CA.

Bennett, M. J. (1979). Overcoming the Golden Rule: Sympathy and empathy. In D. Nommo (Ed.) communication Yearbeok 3, International Communication Association.

Beres, D. U., Arlow, J. A. (1974). Eantasy and identification in empathy. Psychoanalytic Ouarterly, 43, 26-50.

Bernstein, N. (1967). The coordination and regulation of movement. New York: Pergamon Press.

Bion, W. R. (1970). Attention and interpretation. London: Tavistock. 
Birdwhistell, R. L. (1970). Kinesics and context. Philadelphia: University of Pennsylvania Press.

Bohm, D. (1979). Wholeness and the implicate order. New York: Dutton.

Bohm, D. \& Peat, D. F. (1987). Science, erder, and creativity. New York: Bantam Books.

Brain/Mind Bulletin. (1979). Ferguson, M. (ed.). Special Issue.

Brain /Mind Bulletin. (1991a). Ferguson, M. (ed.). 16(4).

Brain/Mind Bulletin. (1991b). Ferguson, M. (ed.). 16(5).

Buber, M. (1948). Between man and man. (Smith, trans.) New York: McMillan.

Capra, F. (1975). The tao of physics. Berkeley: Shambhala.

Capra, F. (1980). The dancing Wu Li masters: An overview of the new physics. Toronto: Bantam Books.

Capra, F. (1982). The turning peint. Toronto: Bantam Books.

Caulfield, H. J. (1984). The wonder of holography. National Geographic, 165(3), 364-376.

Clark, F. V. (1973). Exploring intuition: Prospects and possibilities. Paper for Transpersonal Therapy Institute. Marin County, CA.

Condon, J. C., \& Yousef, F. (1975). An introduction to intercultural communicatien. Indianapolis: Bobbs-Merrill Educational Publishing.

Cooley, C. H. (1922). Human nature and the social order. New York: Charles Scribner's \& Sons.

Coutu, w. (1951). Role-playing vs. role-taking: An appeal for clarification. American Sociological Review, 16, 180-184.

Danish, S. J., \& Kagan, N. (1971). Measurement of affective sensitivity: Toward a valid measure of interpersonal perception. Journal of counseling Psychelogy, 18, 51-54.

Davidson, R. J., Goleman, D. J., \& Schwartz, G. E. (1976). Attentional and affecting concommitants of meditation: A cross-sectional study. Journal of Abnormal Psychelegy, 85 (2), 235-238.

Deikman, A. J. (1982). The observing self. Boston: Beacon Press. 
Deikman, A. J. (1966). Implications of experimentally induced contemplative meditation. Journal of Nervous and Mental Disease, $142(2), 101-116$.

Deutsch, F., \& Madle, R. A. (1975). Empathy: Historic and current conceptualizations, measurement, and a cognitive theoretical perspective. Human Development, 18, 267-287.

Deutsch, J.A., Deutsch, D. (1963). Attention: Some Theoretical Considerations. Rsychological Review, 70, 80-90.

Dewey, J. (1934). Ant as experience. New York: Minton, Balch, \& Co.

Dymond, R. F. (1949). A scale for the measurement of empathic ability. Journal of consulting Psychelegy, 13, 127-133.

Edgerton, F. (ed.). (1951), Bagavad Gita: The song of God. New York: New American Library.

Epstein, M. D. (1984). On the neglect of evenly suspended attention. Journal of Transpersonal Psychology, 16(2), 193-205.

Feshbach, N. D. (1975). Empathy in children: Some theoretical and empirical considerations. Counseling Psychologist, 5. 25-30.

Gabor, D. (1948). A new microscopic principle. Nature, 616, 777-778.

Ghosh, S. (trans, ed. annotated). (1980). The eriginal yoga. New Delhi: Munshiram Manoharlal.

Gill, M. M. and Brenman, M. (1959). Hypnosis and related states: Rsychoanalytic studies in regression. New York: International University Press.

Gladstein, G. A., Brennan, J., Feldstein, J., Ham, H., Kreiser, J., \& Mackrell, S. (1987). Empathy and counseling: Explorations in theory and research. New York: Springer-Verlag.

Goldstein, A. P., \& Michaels, G. Y. (1985). Empathy, development, training and consequences. London: Lawrence Erlbaum Associates.

Goldstein, J. (1986). The experience of insight: A natural unfolding. Santa Cruz: Unity Press.

Goleman, D. (1977). The varieties of the meditative experience. New York: E. P. Dutton.

Goleman, D. (1979, February) - Holographic memory . Esychology Today, $71-83$.

Goleman, D. (1988). The meditative mind. New York: St. Martin's Press. 
Griggs, S. T. (1982). Clairvoyance and the transcendental meditation Sidhi program. Unpublished Dectoral Dissertation, School of Human Behavior, United States International University; San Diego, CA.

Grinberg-zylberbaum, \& Ramos, J. (1987). Patterns of interhemispheric correlation during human communication. International Journal of. Neuroscience, 36, 41-53.

Grof, S. (1983). East and west: Ancient wisdom and modern science. The Journal of Transpersonal Psychology, 15(1), 130-136.

Hall, E. T. (1976). Beyond culture. Garden City, NY: Anchor Press/Doubleday.

Hall, E. T. (1959). The silent language. Garden City, N Y: Anchor Press/Doubleday.

Hastorf, A. H., Schneider, D. J., \& Polefka, J. (1970). Person perception. Reading, MA: Addison-Wesley.

Hampden-Turner, C. (1981). Maps of the mind. New York: MacMillan Publishing.

Heisenberg, W. (1969). Rhysics and beyond. Munich: Piper.

Herbert, N. (1985). Quantum reality: Beyond the new physics. New York: Anchor Press.

Herrigel, E. (1971). Zen in the art of archery. New York: Vintage Books.

Hoffman, M. L. (1976). Empathy, role-taking, guilt and development of altruistic motives. In T. Lickona (Ed.), Moral development and behavior: Theory, research, and social issues. New York: Holt, Rinehart, \& Winston.

Hogan, R. (1969). Development of an empathy scale. Journal of Consulting and clinical psycholegy, 33, 307-316.

Hooper, J. (1978). Interview: Karl Pribram. emni. 129-135, 169-176.

James, W. (1961). The varieties of religious experience. New York: Collier Macmillan.

Johnston, C. (1949). The yega sutras of Patanjali. Albuquerque, NM: Brotherhood of Life.

Johnston, W. (1976). Silent music: The science of meditation. San Francisco: Harper and Row.

Katz, R. I. (1963). Empatby: Its nature and uses. London: CollierMacMilian. 
Keefe, T. (1976). Empathy: The critical skill. Social work, 21, 1014 .

Kohr, R. I. (1977). Dimensionality in meditative experience: A replication. The Journal of Transpersonal Psychology, 2(2), 193203.

Kornfield, J. (1979). Intensive insight meditation: A phenomenological study. Journal of Transpersonal Psychology, 11(1), 41-58.

Korzybski, A. (1958). Science and sanity: An introduction to nenAristotelian systems and general semantics. (4th ed). Lakeville, CT: The International Non-Aristotelian Library Publishing Co.

Kubose, S. K. (1976). An experimental investigation of psychological aspects of meditation. Rsychologia, 19, 1-10.

Kuhn, T. S. (1970). The structure of scientific revolution. (2nd ed). Chicago: University of Chicago Press.

Lafrance, M. (1979). Nonverbal synchrony and rapport: Analysis by the Cross-Iag Panel Technique. Secial Psychelogy Ouarterly, 42(1), 66-70.

Lafrance, M. (1982). Posture, mirroring and rapport. In M. Davis, (Ed.), Interaction and rhythms: Periodicy in communicative behavier. New York: Human Sciences Press.

LaFrance, M., \& Broadbent, M. (1976). Group rapport: posture sharing as a nonverbal indicator. Group and Organizational studies. 1 , 328-333.

Laing, R. D. (1967). The politics of experience. New York: Ballantine Books.

Laszlo, E. (1972). Introduction to systems philosophy. New York: Harper Torchbooks.

Leonard, G. (1981). The silent pulse. New York: Bantam.

Lichtenberg, J. Bornstein, M., Silver, D. (eds.). (1984). Empathy II. Hillsdale, NJ: The Analytic Press.

Lesh, T. V. (1969). The relationship between zen meditation and the development of accurate empathy. Unpublished Doctoral Dissertation, University of Oregon, Eugene.

Lesh, T. V. (1970). Zen meditation and the development of empathy in counselors. Journal of Humanistic Psycholegy, 10(1), 39-74.

Leung, P. (1973). Comparative effects of training in external and external concentration on two counseling behaviors. Journal of Counseling Psychology, 20, 227-234. 
Lipps, T. (1903). Aesthetic esychologie des shonen und der kunst. Hamburg: I Voss.

Lipps, T. (1926). Psychelogical_studies. Baltimore: Williams and Wilkins.

Littlejohn, S. W. (1983). Theories of human communication. Belmont, CA: Wadsworth Publishing.

Livingston, R. B. (1963). Perception and commitment. Bulletin of Atomic Scientists, February.

Lowen, A. (1967). The betrayal of the body. New York: McMillan.

Lundin, R.W. (Bio. ed.). (1984). Reik, Theodore (1888-1969). Encyclopedia of Psycholegy, 3. New York: John Wiley and Sons.

Macarov, D. (1978). Empathy: The charismatic chimera. Journal of Education for Social Work, 14, 86-92.

Mandell, A. J. (ed). 1973). New concepts in neurotransmitter regulation. New York: Plenum Press.

Maupin, E. W. (1965). Individual differences in response to a zen meditation exercise. Journal of Consulting Psychology, 29, 139145 .

McDougal, W. (1908). Introduction to social psychology. Iondon: Methuen.

McLaughlin, J. T. (1975). The sleepy analyst: Some observations on states of consciousness in the analyst at work. Journal of American Psychoanalysis Association, 23, 363-382.

McLuhan, M \& Quentin, F. (1967). The medium is the message. New York: Bantam.

Mead, G. H. (1934). Mind, self and seciety. Chicago: University of Chicago Press.

Means, B. L. (1973). Levels of empathic response. Personnel and Guidance Journal, 52, 23-28.

Murphy, M. \& Donovan, S. (1988). The physical and pychological effects of meditation: A review of contemperary meditation research with a comprehensive bibliography. Oakland, CA: Dharma Enterprises.

Nolte, J. (1988). The human brain: An introduction to its functienal anatomy. St. Louis: C.V. Mosby.

oppenheimer, J. R. (1954). Science and the common understanding. New York: Simon \& Schuster. 
Ornstein, R. E. (1975). On the experience of time. New York: Pelican.

Osis, K., Bokert, E., \& Carlson, M. L. (1973). Dimensions of meditative experience. Jounnal of Transpersonal Psychology, 5(2), 109-135.

Pelletier, K. (1977). The effects of the transcendental meditation program on perceptual style: Increased field independence. In D.W. Orme-Johnson \& J.T. Farrow, eds., Scientific research on the transcendental meditation program: collected papers, I. New York: M.E.R.U. Press.

Pelletier, K. (1978). Towarda science of consciousness. New York: Dell Publishing.

Pelletier, K., \& Garfield, C. (1976). Consciousness:East and West. New York: Harper \& Row.

Prabhavananda, S. (1969). The spiritual heritage of India. Hollywood, CA: Vedanta Press.

Prabhupada, A. C. B. (1974). Srimad Bhagavatam: Third cante. New York: Bhaktivedanta Book Trust.

Pietsch, P. (1981). Shufflebrain. Boston: Houghton Mifflin.

Pribram K.H. (1971). Languages of the brain. Englewood Cliffs, NJ: Prentice-Hall.

Pribram, K. H. (1976). In G. G. Globus, G. Maxwell, \& I. Savodnik, (eds.), Ten problems concerning the structure of consciousness. New York: Plenum Publishing Corporation.

Pribram, K. H. (1978a). Freud's "Project" and the mind brain issue. The Journal of Nervous and Mental Disease, 166(10), 743-752.

Pribram, K. H. (1978b). What the fuss is all about. Re-vision. Summer/Fall.

Pribram, K. H. (1979a, sept.). Mind and brain, psycholegy and neuroscience: The eternal verities. Address at annual meeting of the American Psychological Association. New York City.

Pribram, K. H. (1979b). Transcending the mind brain problem. Zygon. $14(2), 103-123$.

Pribram, K. H. (1980a). Mind, brain, and consciousness: The organization of competence and conduct. In R. J. Davidson, \& J. M. Davison (eds.), The Psychology of Consciousness. New York: Plenum Publishing. 
Pribram, K. H. (1980b). The biology of emotions and other feelings. (245-269). Emotion, theory, research and experience. Academic Press.

Pribram, K. H. (1980C). The orienting reaction: Key to brain representational mechanisms. In H. D. Kimmel (ed.), The orienting reflex in humans. Hillsdale, NJ: Erlbaum.

Pribram, K. H. (1980d). The role of analogy in transcending limits in the brain sciences. Daedalus: Journal of the American Academy of Arts and sciences, $109(2), 19-37$.

Pribram, K. H. (1981). Emotions. In S. B. Filskor \& T. J. Boll (eds.), Handbook of elinical neuropsychology. New York: Wiley.

Pribram, K. H. (1982a). Localization and distribution of function in the brain. In J. Orbach (ed.), Neuropsychology after Lashley. Hillsdale NJ: Erlbaum.

Pribram, K. H. (1982b). The mind brain issue as a scientific problem. In J. Eccles (ed.), Mind and brain: The many-faceted problems. Washington, DC: Paragon House.

Pribram, K. H. (1983a). The brain, cognitive commodities, and the enfolded order. In K. E. Boulding \& L. Senesh (eds.), The eptinum ltilization of knowledge: Making knowledge serve human betterment. Boulder, CO: Westview Press.

Pribram, K. H. (1983b). Brain and the perception of objective reality. In absolute values and the creation of the new world: Proceedings of the lith international conference on the unity of the sciences. New York: ICF Press.

Pribram, K. H. (1984a). Emotion: A neurobehavioral analysis. In K.R. Scherer \& P. Ekman (eds.), Appreaches to Emotion. Hillsdale, NJ: Erlbaum.

Pribram, K. H. (1984b). Mind and brain, psychology and neuroscience, the eternal verities. In S. Koch \& D. E. Leary (eds.), A century of psychology as science. New York: McGraw-Hill.

Pribram, K. H. (1984c, Jan.). Science and the mind brain issue. Essay for International Circle for Research in Philosophy. Unpublished Manuscript. Stanford University, CA.

Pribram, K. H. (1985a). Brain, Behavioral Operants, Cognitive Operations, and Holonomic Transformations. In R. Gandelman (ed.), Perspectives in psychology. Hillsdale, NJ: Erlbaum.

Pribram, K. H. (1985b). "Holism" could close cognition era. Eorum. september. 
Pribram, K. H. (1986). The cognitive revolution and mind brain issues. American Psychologist, 41(5), 507-520.

Pribram, K. H. (1987). Bergson and the brain: A bio-logical analysis of certain intuitions. In P. Papanicolaou \& P. A. Y. Gunter (eds.), The Legacy of Henry Bergson: Toward unification of the sciences, NY: Gordon \& Breach.

Pribram, K. H. (1989a). Brain and perception: Helonomy and structure in figural processing. Unpublished Manuscript.

Pribram, K.H. (1989b). Transcript of personal interview with Dana McCullough. April, 1989. (Available from D. McCullough, 5385 N.W. Jackson School Road. Hillsboro, OR.)

Pribram, K. H. \& Bohm, D. (1978). What the fuss is all about. Revision, Summer/Fall.

Pribram, K. H., \& Bohm, D. (1980). Dromenon, Spring/Summer.

Pribram, K. H., \& Carlton, E. H. (1986). Holonomic brain theory in imaging and object perception. Acta Psychologica, 63, pp. 175310 .

Pribram, K. H., Nuwer, M., \& Baron, R.J. (1974). The holographic hypothesis of memory structure in brain function and perception. In R.C. Atkinson, D.H. Krantz, R.C. Luce \& P. Suppes (eds.), contemporary Developments in Mathematical Psychology. (Vol. II). San Francisco, CA: W.H.Freeman and Co.

Prigogine, I. (1980). Erom being to becoming: Time and complexity in the physical sciences. San Francisco, CA: W.H. Freeman and Co.

Reik, T. Listening with the third ear. (1948). New York: Pyramid Books.

Rokeach, M. (1960). The open and closed mind: Investigations into the nature of belief systems and personality systems. New York: Basic Books.

Rogers, C. (1975). Empathic: An unappreciated way of being. The Counseling Psychologist, 5, 2-10.

Rogers, C. (1983). Ereedom to learn. Columbus, OH: Charles E. Merrill.

Rokeach, M. (1960). The open and clesed mind. New York: Basic Books.

Rosenfield, H. M. (1981). Whither interactional synchrony? In $\mathrm{K}$. Block (ed.), Rrospective issues in infancy research. Hillsdale, NJ: Lawrence Erlbaum Associates. 
Sapir, E. (1958). Culture, language, and the personality. Berkeley: University of California Press.

Schachtel, E. G. (1969). On attention, selective inattention and experience. Bulletin of the Menninger clinic, 33(2), 65-91.

Scheflen, A. E. (1972). Body language and social order: Communication as behavioral control. Englewood Cliffs, NJ: Prentice-Hall.

Scheflin, A. E. (1964). The significance of posture in communication systems. Rsychiatry, 27, 316-331.

Scheler, M. F. (1954). The nature of sympathy, London: Routledge \& Kegan Paul.

Shapiro, D., \& Giber. (1978). Meditation and psychotherapeutic effects: Self-regulation strategy and altered state of consciousness. Archives of General Psychiatry, 35(3), 294-302.

Skinner, B. F. (1938). The behavior of organisms: An experimental analysis. New York: Appleton.

Speeth, K. R. (1982). On psychotherapeutic attention. Journal of Transpersonal Psychology, 14(2), 141-60.

Stein, E. (1964). On the problem of empathy. The Hague, Netherlands: Martinus Mijhoff.

Stein, J. (ed.). (1975). The Random House college dictionary (rev. ed.). New York: Random House.

Strauss, A. (1964). George Herbert Mead on social psychology. Chicago: University of Chicago.

Taimni, I.K. (1986). The science of yoga. Wheaton, IL: The Theosophical Publishing House.

Titchner, E. B. (1910). Text book of psychology. New York: MacMillan.

Truax, C. B., \& Carkhuff, R. R. (1967). Toward effective counseling and psychotherapy. Chicago: Aldine.

Udupa, K. N., Singh, R. H. \& Yadav, R. A. (1973). Certain studies in psychological and biochemical responses to the practice of Hatha Yoga in young normal volunteers. Indian Jounnal of Medical Research, 61(2), 237-244.

Van Nuys, D. (1973). Meditation, attention and hypnotic susceptibility: A correlational study. International Journal of clinical and Experimental Hypnosis, 21(2), 59-69. 
Van Nuys, David. (1971). A novel technique for studying attention during meditation. Journal of Transpersonal Psychology, $3(2)$, 125-133.

Vanderpool, J. P. \& Barratt, E.S. (1970). Empathy: Towards a psychophysiological definition. Diseases of the Nervous System, $21(7), 464-467$.

Van Heel, A. C. S., \& Velzel, C. H. F. (1968). In J. L. J. Rosenfield (trans.), What is light? New York: McGraw-Hill.

Walsh, R. N. (1978). Initial meditative experience: Part II. Journal ef Transpersonal Psychology, 10(1), 1-28.

Walsh, R. N., Goleman, D., Kornfield, J., Pensa, C., \& Shapiro D. (1978). Meditation: Aspects of research and practice. Journal of Transpersonal Psychology, 10(2), 113-133.

Weber, R. (1986). Dialogues with scientists and sages: The search for unity. London: Routledge \& Kegen Paul.

Whorf, B. L., \& Carroll, J. (eds.). (1956). Language, thought and reality: Selected writings of Benjamin Lee Whorf. New York: Technology Press.

Wilber, K. (1977). The spectrum of consciousness. (1977). Wheaton, IL: Theosophical Publishing House.

Wilber, K. (1980). The Atman Project: A transpersonal view of human development. Wheaton, II: Theosophical Publishing House. 
APPENDIX A

THEODORE REIK'S BIOGRAPHY 
THEODORE REIK'S BIOGRAPHY

Reik was born in Vienna in 1888 and received his $P h . D$. degree at the university there in 1912. He became an early follower of Freud and was psychoanalyzed by Karl Abraham, an eminent psychoanalyst. He taught at the Berlin Psychoanalytical Institute before coming to the United States in 1938. His writings, based upon a lifetime of psychoanalytic listening, were intended for the lay person. Reik did not receive an M.D., but became one of the early influential lay analysts. Freud defended Reik's ability to practice psychoanalysis in the absence of medical training.

In his book Listening with the Third Ear, Reik (1948) described the ability of a good analyst to make use of intuitions, sensitivity, and subliminal cues to interpret clinical observations in individual and group psychotherapy. Actually, his ideas were first introduced by Friedrich Nietzsche and later applied by Reik.

Although he believed in many of Freud's psychoanalytical concepts, Reik disagreed with him on matters of love and sex, believing true love is felt most strongly when the loved one is absent. He also took issue with Freud's notion of primary narcissism, which he felt did not exist. Reik wrote on primitive rituals, death rites, and religion as expressions of the fulfillment of unconscious desires. He died in 1969. Source: Lundin, 1984, p. 217. 
APPENDIX B

BRIEF HISTORY OF EMPATHY AS A CONSTRUCT 
BRIEF HISTORY OF EMPATHY AS A CONSTRUCT

The roots of empathy, as a concept of Western culture, have been traced back to plato. The word derives from the Greek word "empatheia," which implies an active appreciation of another person's feeling experience (Astin, 1967, cited in Goldstein \& Michaels, 1985, p 1). In 1897, Lipps (1903, 1926) used the term Einfuhlung--"feeling oneself into"--in his writings which described the capacity for human aesthetic experience (Goldstein \& Michaels, 1985, p. 4). He developed the concept of kinesthetic empathy, or the ability of the body to track and replicate movements observed in others and objects, and outlined four complex elements necessary for empathy to be present. Tichener (1910) later translated this term as empathy

George Mead (1934) enlarged the concept of empathy by adding two elements: the idea of taking the role of the other by putting oneself in the other's place, and a cognitive component, the ability to understand and play back that understanding accurately. Through his work and that of Cooley (1902), Allport (1924), (1908), the concept of interpersonal empathy was strengthened. The role-taking emphasis was further developed by Dymond (1949), Coutu (1951), Moreno (1953), Katz (1963) and Bachrach (1976).

Reik (1948) developed a continuum of empathic process with both an affective and cognitive component, emphasizing the role of attention and the unconscious to achieve empathic insight?. 
Keefe (1976) also developed an affective-cognitive model and stressed the role of accurate feedback to the client. Motor mimicry and microsynchrony were proposed to enhance affective sensitivity (Birdwhistell, 1970); Charny, 1966; Condon and Sander, 1974; Dabbs, 1966, 1969; LaFrance and Broadbent, 1976; Sheflin, 1966). These studies are based on a key assumption, first formulated by James (1860), that felt emotion is the result of neural activity transmitted from the body to the brain (cited in Gladstein \& Michaels, 1965, p. 71). The affective aspects have more recently been elaborated by Danish and Kagan (1971), Rogers (1975), and Truax and Carkhuff (1967).

Three important and inclusive texts--Empathy, its Nature and Uses by Katz (1963), Empathy, Development, Training, and Consequences by Goldstein and Michaels (1985) and Empathy and Counseling: Explorations in Theory and Research by Gladstein, et. al. (1987)--provide comprehensive explorations of the numerous studies and theories relating empathy to psychoanalysis, teaching and counseling. The significant increase in the number of studies included in the latter two texts reflects the escalation of related studies in recent years. With this escalation came confusion regarding the nature and definition of empathy.

Two singular studies explore the combined role of attention and empathic process: Lesh (1970) studied the effect of zen meditation upon accurate empathy, and Bennett (1972) defined and analyzed the role of self-awareness in aesthetic, kinesthetic, object and empathic perception. In The Physical and Psychological Effects of Meditation, 
Murphy and Donovan (1988) cite 19 studies from 1966 to 1985 which conclude that meditation increases empathy and sensitivity (p. 41). These studies are conspicuously missing from the mainstream literature on empathy, but their content is consistent with this thesis.

Sources: Complete information of the several citations within this appendix can be found in Sources Consulted. 
APPENDIX C

DR. KARL H. PRIBRAM'S BIOGRAPHY AND RESEARCH 


\section{DR. KARL H. PRIBRAM'S BIOGRAPHY AND RESEARCH}

Karl Pribram's parents emigrated from Austria when he was 8 years old. He describes himself as a rebellious youth who was expelled from more than one school for either fighting with boys who accused him of being German or challenging the nuns to prove there was a God. Despite his rebellious nature, he achieved academic success, beginning at the University of Chicago, where he finished both a B.S. and an M.D. in 5 years. After a residency in neurosurgery at st Luke's Hospital, he became an instructor in surgery at the University of Tennessee. From there he joined brain scientist Karl Lashley at the Yerkes Laboratory of Primate Biology near Jacksonville, Florida. Pribram became the neurosurgeon on Lashley's research team, helping him write up 30 years of Lashley's studies on the elusive memory trace. It was Lashley who introduced him to the brain/mind enigmas that fueled his curiosity and research interests.

Working as a young neurosurgeon in Jacksonville, Pribram was given a ward of lobotomy patients to oversee. Although lobotomies were a common procedure in psychiatry in the 1940 s and early 1950s, none were performed while he was in charge of the ward. He felt there was not enough known about the brain to justify the widespread practice of lobotomies (Goldstein, 1979, p. 76).

He did, however, do extensive research on laboratory animals. One of his key findings was that the frontal lobes were tied to the limbic system, especially the amygdala. 
Pribram directed Yerkes Labs briefly after Lashley's retirement. Then, in 1948, he went to Yale where he held appointments in the departments of Psychiatry and Psychology, and was also Director of the Institute for Living in Hartford. He stayed for 10 years, pioneering research on the limbic system. At that time the limbic area was thought of as an olfactory brain, mainly involved in the sense of smell. Pribram was the first to demonstrate that the system was far more complex, and that visceral as well as olfactory information was processed here (Flaherty, cited in Pribram, 1985a, pp. 59-60).

In 1959 Pribram began his research at Stanford in the departments of Psychology and Psychiatry where he was Head of the Neuropsychology Laboratories. In 1960 Pribram challenged the prevailing wisdom in a book that he wrote with George A. Miller and Eugene Galanter called Plans and the Structure of Behavior. Until then, Miller and Pribram had been staunch behaviorists, but in the book they declared that the assumptions underlying the behaviorist school were mistaken. Pribram's contribution was the explanation that the brain cells did not work as a simple reflex-arch, but rather were part of an interactionist-feedback circuit. They renamed themselves "subjective" behaviorists and their shift gave impetus to the cognitive revolution that swept first through human-learning research and then animal-learning research. At Stanford, Pribram and his students carried on an extensive series of experiments analyzing the function of the amygdala, demonstrating its function as an important nodal point in the network controlling fighting, fleeing, feeding and sex. In 1962 he was given a lifetime research award by the 
National Institutes of Health (Flaherty, cited in Pribram, 1985a, pp. $59-60)$

His book Languages of the Brain, published in 1971, signaled his departure from the cognitist perspective. The text continued his development of the feedback model of behavior and introduced a significant new development, the consideration of the synaptic junction as the locus of neurobehavioral flexibility. He related the interaction among neural slow potentials that takes place at the synapse to the concepts of holography. Pribram's own emphasis was the visual system, but he brought together research from several laboratories demonstrating the likelihood that all senses operate in the transform domain. He postulates the Fourier Transform as the underlying mathematical principle, inviting research challenges to his theory. (Flaherty, cited in Pribram, 1985a, p. 60).

At Stanford he also took to an analysis of music, extending the Chomskian analysis of music first offered by Leonard Bernstein. Concurrently, he undertook a Navy grant to work with Bob Isaacson on the analysis of neural mechanisms of learning and memory (Flaherty, cited in Pribram, 1985a, p. 62).

The popular press has oversimplified Pribram's work, attributing to Pribram claims about the brain that Pribram himself disclaims. Of all popular writing about his work by lay persons, Pribram attributes the most insightful explanations of the implications of his work to Marilyn Ferguson, editor of the Brain/Mind Bulletin in Los Angeles (Pribram, personal communication, April, 1989). 
Pribram left Stanford in 1990 to pursue his interests in human perception at Radford University in Virginia as an Eminent Scholar. This university is currently constructing a laboratory for his work, and he has gathered a group of scientists with similar goals, among them Peter Kugler, William Hudspeth and Lawren Gerbrant (L.Gerbrant, personal communication, April 3, 1991).

The scope and magnitude of Dr. Pribram's interests and research are impressive. He has served on the editorial board of journals as diverse as Human Motivation, Neuroscience Research, Journal of Autism and Developmental Disorders, Journal of Mental Imagery, cognition and Personality, Journal of Mathematical Biology, and the Journal of Euman Movement Studies (Flaherty, cited in Pribram, 1985a, p. 59).

Pribram has published over 150 review and theoretical papers, over 100 research papers, 13 books and monographs, and numerous book reviews and commentaries. The common thread throughout his work is the relationship between human consciousness and the brain.

Source: Information was generalized from four sources: (a) Pribram, 1985a, pp. 59-62; (b) Goleman, 1979, p. 76; (c) Pribram, 1989, April, 1989; and (d) L Gerbrant, Personal communication, April 3, 1991. 


\section{APPENDIX D}

CHRONOLOGY OF THE HOIONOMIC BRAIN METAPHOR 


\section{CHRONOLOGY OF THE HOLONOMIC BRAIN METAPHOR}

1714--Gottfried Wilhelm Von Leibniz, discoverer of integral and differential calculus (including the Fourier Transform), said that a metaphysical reality underlies and generates the material universe. Space-time, mass and motion of physics and transfer of energies are intellectual constructs.

1902--William James proposed that the brain normally filters out a larger reality.

1905--Albert Einstein published his theories. Space and time are related constructs.

1907--Henri Bergson said that the ultimate reality is a vital impulse comprehensible only by intuition. The brain screens out the larger reality.

1929--Alfred Whitehead, mathematician and philosopher, described nature as a great expanding nexus of occurrences not terminating in sense perception. Dualisms such as mind/matter are false; reality is inclusive and interlocking.

1929--Karl Lashley published his great body of research demonstrating that specific memory is not to be found in any particular site in the brain but is distributed throughout. Pribram later worked with Lashley.

1947--Dennis Gabor employed Leibniz' calculus to describe a potential three-dimensional photography: Holography.

1965--Emmitt Leith and Juris Upatnicks announced their successful construction of holograms with the newly invented laser beam.

1969--Karl Pribram, who had worked with Lashley as a neurosurgeon, proposed that the hologram was a powerful model for brain processes.

1971--Physicist David Bohm, who had worked with Einstein, proposed that the organization of the universe may be holographic.

1971--Pribram synthesized his theories and Bohm's in a German publication on Gestalt psychology.

1977--Pribram speculated on the unifying metaphysical implications of the synthesis.

Source: Brain/Mind Bulletin, 1979, p. 4. 
APPENDIX E

HOLOGRAM MODEL AND EXPLANATION 


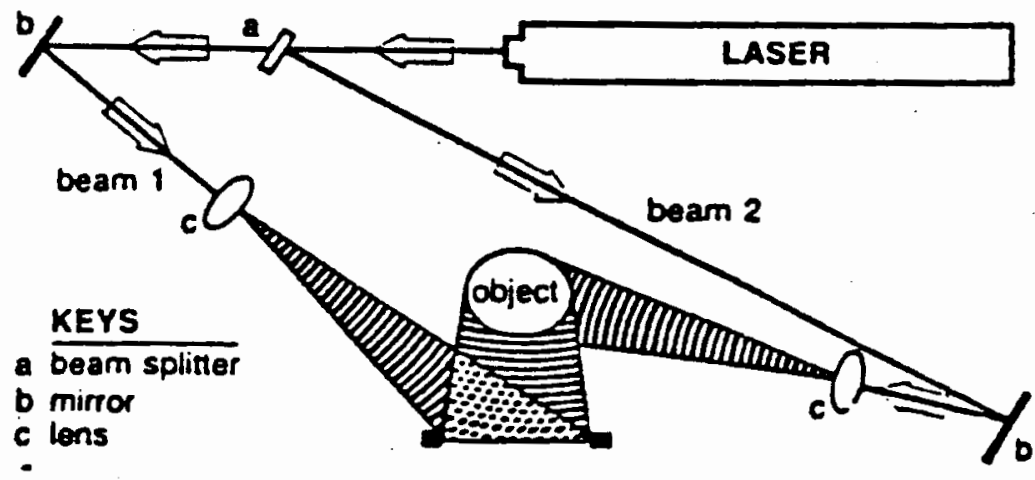

Drawing Illustrating how ahologram is constructed from coherent light.

After the laser's original beam is split (at point "a"), one part is deflected toward the film plate and the other is bounced off the object. When the beams reconverge, they form a light interference pattern that is recorded. The image is reconstructed when a second beam: is reflected off the plate and diffused in the same pattern.

Explanation: If you drop two pebbles into a pond, concentric circles or waves radiate out from each. When the two sets of waves run into each other, they form an interference pattern. Where the crests of waves meet, they make a wave that is twice as high; if a wave meets a trough, the two will cancel each other out to form a flat patch; if two troughs meet, they will make a deeper trough. Light waves produce the same complex mix of interference patterns when they intersect. Like the 
pebbles in the pond, a laser--the purest form of light available--sends out a beam of light waves in one frequency. When two laser beams meet, they produce an interference pattern of light and dark. If one of the beams is reflected off an object, such as a face, and then strikes a photographic plate, the plate will record the interference pattern, thus storing an image of the face. In an ordinary light, the plate looks a uniform silvery gray, but when the right frequency of light strikes the plate, the original, an interference pattern is set up, creating a holographic image. We see a replica of the face, projected away from the plate, that appears three-dimensional in that the viewer can see different sides of it as he changes position Source: Excerpt from Goleman, 1979, pp. 71-83. 
APPENDIX F

FOURIER TRANSFORM:

HISTORY, THEOREM AND EXPLANATION 


\section{FOURIER TRANSFORM : \\ HISTORY, THEOREM AND EXPLANATION}

In 1798 Joseph Fourier accompanied Napoleon on his Egyptian adventure. He served for 2 years as governor of southern Egypt and in 1801 returned to France. Later, he gave to the world the Fourier Theorem, which he is presumed to have discovered during his travels. It provided the key to wave language and became the foundation stone of all wave-based sciences, including communications theory, modern optics, sound reproduction, oceanography, and quantum theory.

Fourier developed his waveform language to deal with heat waves. Heat, the motive power behind the burgeoning industrial revolution, was an exciting mystery to the 19 th century physicists and engineers. Lord Kelvin, the dean of English physicists, described La Theorie Analytique de la chaleur, Fourier's study of the flow of heat, as "a great mathematical poem." Fourier's Theorem states that any wave can be written as a unique sum of sine waves.

The sine wave is a kind of undulatory archetype; its curvey profile is what most people have in mind when they visualize a wave. Vibrating strings and ripples in a pond are shaped each moment like sine waves. To see a sine wave standing still, look sideways at a stretched spring (or any other helix).

Physicists like these waveforms because when they put a sine wave into any linear system, a similar sine wave always comes out. Linear systems change a sine wave's amplitude and phase, but they never change 
its sinusoidal shape. Mathematicians like sine waves because no matter haw many times they differentiate them, the result is always more sine waves. After listing the special attributes of this popular wave form, E. A. Guillemin celebrated the sine wave in words unusually colorful for an electrical engineer: "The sine wave is singled out as the one that shall forever be king and ruler."

The mathematics that explains the process is a form of calculus. It is used to break up a compound wave into its constituent components and, conversely, to generate a compound wave from its basic components. It can be applied to any and all wave forms and frequencies. Its unique attribute is that the identical equation covolves and devolves itself. Imaging a wave, $W$, stretched out in space. Wave $W$ is not necessarily oscillatory; it may take any shape whatever. Fourier's theorem says that wave $W$ may be written as a sum of sine waves with various spatial frequencies $k$, amplitudes $a$, and phases $p$ Each word in Fourier's sine wave language is a sine wave with a different value for $k, a$, and $p$. Translating a wave into its sine wave words is called fourier analysis. Wave $W^{\prime} s$ Fourier analysis looks schematically like this:

$$
w=(k a p) 1 \oplus(k a p) 2 \oplus(k a p) 3 \odot(k a p) 4 \Theta
$$

The particular sine waves which describe wave $W$ are called its Fourier spectrum, or sometimes its vibration recipe. Each vibration recipe is unique: There is oniy one way to translate a wave into this sine wave 
language. The gist of Fourier's important discovery is that sine waves form a universal alphabet in terms of which any wave can be written. Scientists have analyzed the sound waves produced by various musical instruments in terms of Fourier's sine wave alphabet. Even when sounding the same note, each instrument produces its own particular "tone color"--a difference reflected in its vibration recipe. Each instrument leaves a unique "Fourier fingerprinting" in the air. Just as a wave can be broken up into sine waves, so the same wave can be put together out of sine waves, an operation called "Fourier synthesis."

Source: Herbert, 1985, pp. 79-80. 
APPENDIX G

HOLONOMIC ENFOLDING-UNFOLDING SCHEMATIC 
HOLONOMIC ENFOLDING-UNFOLDING SCHEMATIC

\section{Energy \\ (Change)}

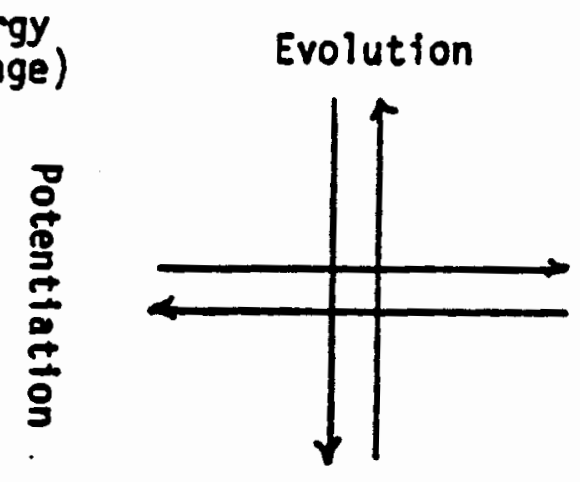

Momentum (Inertia)
Materfalization

\section{Entropy \\ (Time)}

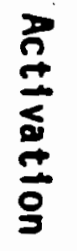

\section{Location (soace)}

Karl Pribram's visual schematic of the potentializing and realizing attributes of holonomic transforming.

The Gabor, Fourier-like transform does have a special virtue. By way of this transform the microstructure of matter can be shown to partake of the same structural properties as the microstructure of memory. Heisenberg (1969) demonstrated that one can measure the . momentum of an event or its localization, but not both simultaneously. This is the famous uncertainty principle. Momentum is a measure of potential energy; location places that event in space/time. Henry Stapp (1965) has shown that the measures of energy/momentum and of space/time are Fourier Transforms of one another. We are thus faced with a fourfold relationship in which energy (E) represents potential for change; momentum $(p)$ the inertial resistance to change; space ( $x$ ) the spatial dimension; and time ( $t$ ) the time dimension. In a sense, energy thus 
reflects a prototemporal and momentum a protospatial dimension. Further, Einstein's relativity equation--E=mc2-- indicates that measures on matter (i.e., mass, m) are transforms of measures on energy and the speed of light--E/C2). Thus neither energy nor light are, in a strict sense, matter (i.e., they do not possess rest mass) although they interact with matter.

The four-fold relationship between energy, momentum, space, and time provides some interesting insights. When one proceeds from the potential domain of energy and momentum to that of space and time, one is actualizing the potential. When one proceeds in the reverse direction, one enfolds, by virtue of the Fourier Transform, space and time into the frequency domain. The enfolding process empowers: Power is a measure of the energy at a given frequency.

Proceeding from the domain of energy and time to the domain of momentum and space leads to materialization, since it is masses that display momentum and location. How then do we characterize the reverse, i.e., proceeding from the domain of momentum and space to that of energy and time? Measures of change in time are measures of efficiency, measures which make up the subject matter of thermodynamics. The measure of efficiency is the reciprocal of entropy, negentropy. Negentropy has been related to measures of information (the number of alternative events to be specified). The structuralizing processes which negate the entropic thrust of the physical universe are vital (i.e., life-giving), mental (i.e., mnemic, based on memory), and spiritual (i.e., informing). 
Shades of Bergson's elan vital? Perhaps, and why not? Science is the search for beauty in nature. The relationships displayed above give precision to what appeared to be a vague mystical conception, a fantasy. But according to Bergson's own rules, a fantasy it was not. The conception of an elan vital turned out to be intuitive because by dividing the subdividing, the intuiton became intelligible. And, as a personal subscript, no one can be more surprised than I. Source: Excerpts from Pribram, 1987, p 20-21, 23. 